\title{
A REIMBEDDING ALGORITHM FOR CASSON HANDLES
}

\author{
ŻARKO BIŽACA
}

\begin{abstract}
An algorithmic proof of Freedman's Reimbedding Theorem [F2] is given. This reimbedding algorithm produces an explicit description of an imbedded Casson tower with seven levels inside an arbitrary Casson tower with six levels. Our approach is similar to Freedman's original idea, but we also make essential use of the grope technology from [FQ]. The reimbedding algorithm is applied to obtain an explicitly described Casson handle inside an arbitrary sixlevel tower (Theorem A), a description of a family of exotic Casson handles (Theorem B) and an explicitly constructed exotic $\mathbf{R}^{4}$.
\end{abstract}

\section{INTRODUCTION}

In his 1982 paper [F2], M. H. Freedman proved that in any six-level Casson tower one can embed a seven-level Casson tower which has the same attaching circle. The numbers were reduced by R. Gompf and S. Singh in [GS] to an embedding of a six-level tower inside of any five-level tower. Both proofs guarantee the existence of a Casson tower of one level more then the original tower and by iterating the procedure they imply the existence of a full Casson handle inside the original Casson tower. In [G3], Gompf has described an explicit embedding of a union of an eight-level Casson tower and the 4-ball into $\left.\overline{\mathrm{CP}}^{2} \#\left(\#_{m} \mathbb{C P}\right)^{2}\right)$ that represents a second homology class of the manifold which cannot be represented by a smoothly embedded sphere. Consequently, any Casson handle embedded into this eight-level Casson tower cannot be diffeomorphic to the standard open 2-handle. However, both proofs of the existence of an embedded Casson handle in a Casson tower rely on general position arguments and fundamental group calculations and therefore do not describe the embedded Casson handles explicitly.

The purpose of this work is to produce a concrete description of the Casson handles obtained by Freedman's Reimbedding Theorem (Theorem A), and in particular, a description of a family of exotic Casson handles (Theorem B) and an explicit construction of an exotic $\mathbb{R}^{4}$ (subsection 1.0.4).

This paper is divided into two sections. The first section contains an algorithm for a construction of a seven-level Casson tower inside an arbitrary sixlevel tower. It is a modified version of Freedman's or Gompf and Singh's proof

Received by the editors January 13, 1993.

1991 Mathematics Subject Classification. Primary 57M99, 57N13.

Key words and phrases. Casson handles, Casson towers, exotic Casson handles, exotic $\mathbf{R}^{4}$, reimbedding algorithm, Freedman's Reimbedding Theorem, capped gropes, Casson fingers, singular Norman trick. 
of the Reimbedding Theorem and it uses the "grope" technology. Section 1.0 contains the main results and applications. Also, subsection 1.0.5 outlines some additional applications of the reimbedding algorithm that will appear in [B1] and [B2]. Section 1.1 contains a short outline and strategy of the construction of an embedded seven-level tower.

The reimbedding algorithm from the first chapter is used in the second chapter to estimate the numbers of the kinks on the embedded seven-level tower. These estimates are used to estimate the number of kinks of an embedded Casson handle, as claimed in Theorem A. The obtained numbers are by no means the smallest possible and there is no reason to believe that this algorithm is in any sense the most effective or the simplest one. The disappointed reader is encouraged to find her or his own estimates.

All figures are presented at the end of this paper.

I would like to express my gratitude to my advisor at Notre Dame, Professor Laurence $\mathbf{R}$. Taylor, for advice, help and patience during my studies and work on this paper. Also, I am grateful to the referee for many helpful suggestions.

\section{THE REIMBEDDING ALGORITHM}

Terminology. We will follow the conventions from [F2] and [GS] in denoting an $n$-level Casson tower by $T_{n}$, its core by $C_{1-n}$, and the core discs from the $j$ th level by $C_{j}$. If needed, we will index towers, cores and levels of different Casson towers with superscripts. The link calculus ([K1] or [F2]) description of a Casson tower will be used in most pictures. We replace 1-handles by scooped out 2-handles, but by a standard abuse of notation we continue to call them "1-handles". Capped gropes have been described in [FQ, Chapter 2]. By $c G_{i}^{n}$ we denote the " $i$ th $n$-stage capped grope", i.e., the one which has $n$ "stages" of "the body" $G_{i}$, where the subscript $i$ comes from some ordering of capped gropes (see [FQ, p. 36]). Caps of the capped grope $c G_{i}^{n}$ are denoted by $c_{i}^{ \pm}$, and by $c_{i}^{+}$or $c_{i}^{-}$, where the signs came from an ordering of each pair of "standard generators" for the fundamental group of the top stage of the body of the grope $c G_{i}^{n}$ (see [FQ, section 3.6]). We will also use the notions of "contraction" and "pushing off" from [FQ].

We will always view Casson towers and Casson handles as ordered pairs with the manifold itself as the first component of the pair, and the attaching area as the second component. The attaching area of a Casson tower $T$ is the image of an embedding of the solid torus $S^{1} \times D^{2}$ inside the boundary of the tower and is denoted by $\partial^{-} T$. Accordingly, $\partial^{+} T$ denotes $\partial T-\operatorname{int}\left(\partial^{-} T\right)$. Any Casson handle can be constructed as an infinite union of the one-level Casson towers from which the $\partial^{+}$-part of the boundaries have been removed. Consequently, the whole boundary of a Casson handle is its attaching area.

We assume that all maps between these pairs are identity on the cores of the embedded solid tori from the second components. Any embedding of a Casson tower $T_{1}$ into a Casson tower $T_{0}$ will the property $\partial^{-} T^{1}=\partial T^{0} \cap T^{1} \subset \partial^{-} T^{0}$.

\subsection{Main results.}

1.0.1. Freedman's Reimbedding Theorem. Our algorithm for the embedding of a Casson handle in a six-level Casson tower contains another proof of the following Freedman Reimbedding Theorem. 
The Reimbedding Theorem (cf. [F2, Theorem 4.4]). For each six-level Casson tower $T_{6}^{0}$, there is an embedding of a seven-level Casson tower $T_{7}^{1}$ in $T_{6}^{0}$ with the following properties:

(a) $C_{1}^{1}=C_{1}^{0}$ and

(b) $\pi_{1}\left(T_{6}^{0}-T_{7}^{1}\right) \rightarrow \pi_{1}\left(T_{6}^{0}-C_{1}^{0}\right)$ is an isomorphism.

As an intermediate result, we will construct another six-level tower inside $T_{6}^{0}$ that will prove the following theorem.

The Big Reimbedding Theorem (see Theorem 4.3 in [F2] or [GS, p. 306]). In each six-level Casson tower $T_{6}^{0}$ there is a six-level Casson tower $T_{6}^{1}$ such that:

(a) $C_{1}^{1}=C_{1}^{0}$,

(b) $\pi_{1}\left(T_{6}^{1}\right) \rightarrow \pi_{1}\left(T_{6}^{0}\right)$ is a trivial map and

(c) $\pi_{1}\left(T_{6}^{0}-T_{6}^{1}\right) \rightarrow \pi_{1}\left(T_{6}^{0}-C_{1}^{0}\right)$ is an isomorphism.

The " $T_{6}^{1}$ " is going to be the first six-levels of a seven-level tower embedded in the tower $T_{6}^{0}$. The standard statement of the Big Reimbedding Theorem involves an embedding of a five-level Casson tower into an arbitrary five-level tower. This sharper version can also be deduced from our construction, roughly by shedding off the first levels of the towers but it will be of no importance. Repeated applications of the construction of an embedded six-level tower in the previous one will be used to construct a system of neighborhoods of the end of an exotic $\mathbb{R}^{4}$.

1.0.2. Input tower and an embedded Casson handle. Before we start the construction of a seven-level Casson tower in the input tower $T_{6}^{0}$, we are going to symmetrize the input tower by making all the branches equal: for each level, except the first one, we find the maximal number of positive or negative kinks (which we denote by $x_{n}$ ) and change $C_{2-6}^{0}$ by adding extra kinks to its discs such that all the discs forming the $n$th level have the number of positive and the number of negative kinks equal to $x_{n}$. Also, we assume that numbers $x_{2}$, $\ldots, x_{6}$ form an increasing sequence by adding more kinks if needed. Adding a kink is described in [K2, p. 72] and its link calculus description is the change from Figure 1(a) to Figure 1(b). The input of our algorithm is now a six-level symmetric Casson tower (still denoted $T_{6}^{0}$ ) which can be completely described by the following seven numbers: $x_{2}, \ldots, x_{6}$ and the pair of nonnegative numbers $(p, n)$ which consists of the number of positive kinks and the number of negative kinks of the disc in the first-level, or, in another words, the number of positive and negative branches of $T_{6}^{0}$. Our output will be a Casson tower $T_{7}^{1}$ which will have the same $C_{1-4}$ as the symmetrized input, and the discs in the higher levels will have the same intersection numbers throughout the level. Of course, for different levels we will have different numbers: $x_{5}^{\prime}, x_{6}^{\prime}$ and $x_{7}^{\prime}$.

We will use this algorithm repeatedly to obtain a sequence $T_{6}^{0} \supset T_{7}^{1} \supset$ $T_{8}^{2} \supset T_{9}^{3} \cdots$ and finally a Casson handle embedded in $T_{6}^{0}$. We start by applying the reimbedding algorithm to embed $T_{7}^{1}$ in $T_{6}^{0}$. The second time, we use the algorithm on each branch of $T_{7}^{1}$ separately. Each branch is symmetric in the above sense and they all can be described by the same set of numbers: $x_{2}$, 
$x_{3}, x_{4}, x_{5}^{\prime}, x_{6}^{\prime}$ and $x_{7}^{\prime}$, so the algorithm will embed diffeomorphic seven-level towers in the branches. These seven level towers and the original first level will be all glued together to form a Casson tower with eight levels, $T_{8}^{2}$. Continuing in this manner we obtain a sequence of embeddings of Casson towers as above. Because each new embedding changes only the two topmost levels of the previously embedded tower, we can form the following infinite union: from the first four levels of $T_{6}^{0}$ we remove the $\partial^{+}$-part of the boundary, then add the fifth level of $T_{7}^{1}$, the sixth level of $T_{8}^{2}$, and so on, with all $\partial^{+}$-parts of the boundaries removed. The resulting union is a Casson handle embedded in $T_{6}^{0}$ which is specified up to diffeomorphism by the pair $(p, n)$ and a sequence of numbers $\left\{y_{k}\right\}_{k=2}^{\infty}$. Again, $p$ is the number of positive kinks and $n$ the number of negative kinks on the disc in the first level and, for each $k>1, y_{k}$ is the number of positive kinks and the number of negative kinks on each disc in the $k$ th level.

There are two reasons for symmetrizing towers. A Casson handle is defined by an rooted, finitely branching infinite signed tree. An easy way to define one is to specify that for each vertex on the $n$th level there are $2 y_{n}$ branches, half of them with the + sign and half with the - sign (i.e., all discs of the $n$th level have $y_{n}$ positive and $y_{n}$ negative self-intersections). Another reason is that new levels of a Casson tower are formed by adding 'kinky handles', but, in general, the framings of the attaching circles of the kinky handles will be different from those induced by self-plumbings of trivial disc bundles (see [GS, p. 283] or [F2, p. 377]). Recall that if $a$ is an attaching circle of a kinky handle $K$, then the gluing of 2-handles onto the kinky handle $K$, over the "standard loops" with their "correct framings", changes the pair $(K, a)$ to $\left(D^{4}\right.$, unknot). The "correct framing" of the attaching circle $a$ is the one that results in the O-framing of the unknot. Another framing comes from removing the kinks by piping them to geometrically dual spheres with the trivial normal bundles. Then, the resulting framing of $a$ is the one coming from the self-plumbings. If the numbers of positive and negative kinks are equal, these two framings are identical.

Figure 2 shows a branch of an initial tower $T_{6}^{0}$ after the symmetrization. Possibly, some 1-handles of the sixth level are produced by introduction of pairs of complementary 1- and 2-handles. The added 2-handles of the pairs are not shown in the figure.

Iteratively applying the reimbedding algorithm, we can construct a Casson handle in a given six-level Casson tower.

Theorem A. If $T_{6}^{0}$ is a symmetrized Casson tower with six levels that has $x$ positive and $x$ negative kinks on every disc except the one on the first level, then there is an embedded Casson handle $C H$ in $T_{6}^{0}$ with the same first four levels as $T_{6}^{0}$ and all discs of the nth level have $y_{n}$ positive and $y_{n}$ negative kinks, where $y_{n}$ is

(1) $y_{i}$ is equal to $x$ for $i=2,3,4$;

(2) $y_{5}=(10 x)^{2}$;

(3) $y_{6}$ is $(10 x)^{10^{(10 x)^{10}}}$ and 


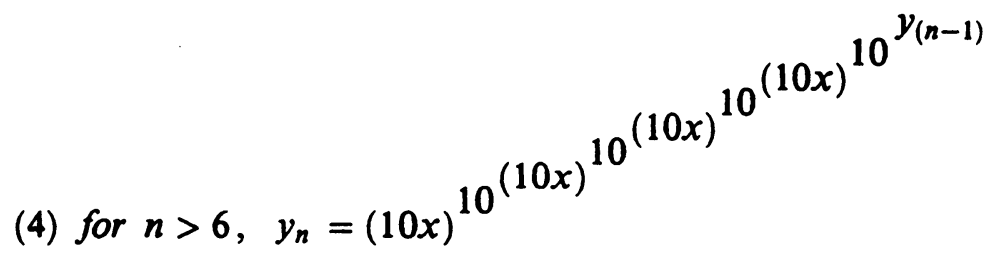

Remark 1.0.2. For a nonsymmetrized six-level input tower the embedded Casson handle has only the same first level disc as the input tower. The variable $x$ figuring in the definition of the embedded Casson handle can be taken to be an upper bound of the numbers of positive and the numbers of the negative kinks of the discs in $C_{(2-6)}^{0}$, the upper levels of the input tower. The sequence $\left\{y_{n}\right\}_{n=2}^{\infty}$ remains the same.

1.0.3. Exotic Casson handles. Let $V=B^{4} \cup T_{8}$ where $T_{8}$ is attached to the 4-ball via an identification of the canonical framing of the attaching circle of $T_{8}$ and the -1 framing of the unknot in $\partial B^{4}$. In [G3], Gompf constructs a smooth embedding of $V$ in $M_{m}=\overline{\mathbb{C P}}^{2} \#\left(\#_{m} \mathbb{C} P^{2}\right)$ for "sufficiently large" $m$, so that the map $\mathbb{Z}=H_{2}(V ; \mathbb{Z}) \rightarrow H_{2}\left(M_{m} ; \mathbb{Z}\right)$ sends a generator to $3 e_{0}+e_{1}+\cdots+e_{8}$, where $e_{i}, 0 \leq i \leq m$, are the evident homology generators.

Lemma. Let $X \subset V$ be any subset such that $H_{2}(X ; \mathbb{Z}) \rightarrow H_{2}(V ; \mathbb{Z})$ is onto. Then there does not exist a smooth embedding, $S^{3} \subset V-X$.

Proof. Suppose that there is such an embedding $S^{3} \subset V-X \subset M_{m}-X$. Then we could replace by a 4 -ball the component of $M_{m}-S^{3}$ that contains $X$. The intersection form of the resulting smooth closed manifold would be equal to $E_{8} \oplus(m-9)\langle+1\rangle$ and such a manifold does not exist by Donaldson's work [D].

One application, known to Gompf, is that any Casson handle embedded in $T_{8}$ must be exotic: if it were standard, we could take $X=S^{2}$ in the Lemma and the $S^{3}$ would be the boundary of the total space of the normal disc bundle. Use Theorem A to construct a Casson handle in $T_{8}$ and note that since kinkier Casson handles embed in the less kinky ones, we have proved

Theorem B. Let CH be any Casson handle which has at least one negative kink on the discs of the first level, and a branch over one of these kinks with the following description:

(1) each disc from the second, third and fourth levels of the branch has at least one negative and one positive kink,

(2) each disc on the fifth level has at least 100 positive and 100 negative kinks and

(3) for $n>5$, all discs of the nth level of the branch have at least $y_{n}$ positive and $y_{n}$ negative kinks, where $y_{n}$ is

(a) $y_{6}$ is $10^{10^{10^{10}}}$ and

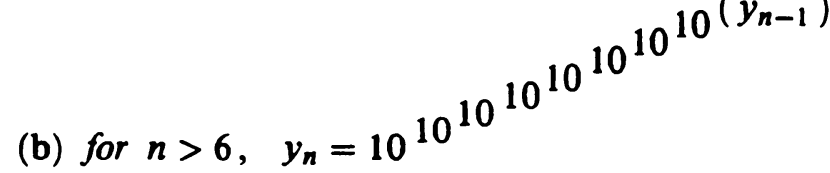


Then, there is no diffeomorphism between the $C H$ and $D^{2} \times \mathbb{R}^{2}$, extending a diffeomorphism between $\partial^{-} C H$ and $S^{1} \times \mathbf{R}^{2}$.

1.0.4. Algorithmic description of an exotic $\mathbb{R}^{4}$. To construct an exotic $\mathbf{R}^{4}$, recall that $T_{8}$ embeds in the standard 2-handle, and hence $V \subset \overline{\mathbf{C P}}^{2}$. Let $T^{0} \subset T_{8}$ denote a symmetrized sixth-level tower inside $T_{8}$. By induction, define $T^{i+1} \subset T^{i}$ by applying the Reimbedding Theorem to $T^{i}$ and then discarding the seventh level. Define $N_{i}=B^{4} \cup T^{i}$ via an identification of the canonical framing of the attaching circle of $T_{8}$ and the -1 framing of the unknot in $\partial B^{4}$ as before. If we shrink the 4-ball a little each time, we get a sequence of embeddings, $N_{i+1}$ embedded in the interior of $N_{i}$ : let $X=\bigcap_{i=1}^{\infty} N_{i}$. By construction $X \subset N_{i} \subset V \subset \overline{\mathbb{C P}}^{2}$. We show

Example. $\overline{\mathbf{C P}}^{2}-X$ is an exotic $\mathbf{R}^{4}$.

Proof. There is an immersion $S^{2} \rightarrow V$ whose image consists of a slice of the unknot, $B^{2} \subset B^{4}$, and the first level core disc of the tower, $C_{1}$. This map factors through each $N_{i}$, and hence through $X$. The maps induced on $H_{2}(; \mathrm{Z})$ by $S^{2} \rightarrow V$ and $V \rightarrow \overline{C P}^{2}$ are isomorphisms, so the Lemma applies to show $\overline{\mathbf{C P}}^{2}-X$ is not diffeomorphic to $\mathbf{R}^{4}$. If we can show that $\overline{\mathbf{C P}}^{2}-X$ is simply connected, simply connected at infinity, and that one has $\mathrm{H}_{2}\left(\overline{\mathrm{CP}}^{2}-X ; \mathrm{Z}\right)=0$ we are done by Corollary 1.2 of Freedman [F2].

To fix some notation, recall $B^{4} \cap T^{i}$ is a solid torus: let $F^{2} \subset T^{i}$ denote the boundary torus. With respect to the solid torus, we have a meridian, $\mu$, in $H_{1}(F ; Z)$; note $F^{2} \subset T^{i}-T^{i+1} \subset T^{i}-C_{1}$ and the induced map $\pi_{1}\left(F^{2}\right) \rightarrow$ $\pi_{1}\left(T^{i}-C_{1}^{i}\right)$ has kernel $\mathbb{Z}$. Let $\lambda$ be a generator. Note that by part (c) of the Big Reimbedding Theorem, $\lambda$ dies in $\pi_{1}\left(T^{i}-T^{i+1}\right)$. Note $\lambda$ and $\mu$ generate $\pi_{1}\left(F^{2}\right)$. Finally, the kernel of the map $\pi_{1}\left(T^{i}-T^{i+1}\right) \rightarrow \pi_{1}\left(T^{i}\right)$ is the normal closure of $\mu$, since this is clearly true for the map $\pi_{1}\left(T^{i}-C_{1}\right) \rightarrow$ $\pi_{1}\left(T^{i}\right)$.

A simple van Kampen argument shows that

(a) $\pi_{1}\left(T^{i}\right) \rightarrow \pi_{1}\left(N_{i}\right)$ is an isomorphism.

Furthermore, $\quad N_{i}-N_{i+1}=\left(B^{4}-B^{2} \times B^{2}\right) \cup_{F^{2}}\left(T^{i}-T^{i+1}\right)$, where $B^{2} C$ $B^{4}$ is the standard slice for our unknot in $\partial B^{4}$ and $B^{2} \times B^{2}$ is a tubular neighborhood of it. The map $\mathbb{Z} \oplus \mathbb{Z}=\pi_{1}\left(F^{2}\right) \rightarrow \mathbb{Z}=\pi_{1}\left(B^{4}-B^{2} \times B^{2}\right)$ is just subtraction, since we are attaching with framing -1 , and so the van Kampen theorem shows that $\pi_{1}\left(T^{i}-T^{i+1}\right) \rightarrow \pi_{1}\left(N_{i}-N_{i+1}\right)$ is onto with kernel normally generated by $\mu$. Hence the map

(b) $\pi_{1}\left(N_{i}-N_{i+1}\right) \rightarrow \pi_{1}\left(N_{i}\right)$ is an isomorphism.

By comparing $N_{i}-N_{i+k+1}=\left(N_{i}-N_{i+k}\right) \cup\left(N_{i+k}-N_{i+k+1}\right)$ with $N_{i}=$ $\left(N_{i}-N_{i+k}\right) \cup N_{i+k}$ and using (b) above, we see that the map $\pi_{1}\left(N_{i}-N_{i+k}\right)$ $\rightarrow \pi_{1}\left(N_{i}\right)$ is an isomorphism for $k \geq 1$. By a limit argument,

(c) $\pi_{1}\left(N_{i}-X\right) \rightarrow \pi_{1}\left(N_{i}\right)$ is an isomorphism.

By part (b) of the Big Reimbedding Theorem and (a) above, the map

(d) $\pi_{1}\left(N_{i+1}\right) \rightarrow \pi_{1}\left(N_{i}\right)$ is 0

so $\pi_{1}\left(N_{i+1}-X\right) \rightarrow \pi_{1}\left(N_{i}-X\right)$ is also 0 . Hence $\overline{\mathbb{C P}}^{2}-X$ is simply connected at infinity.

By comparing $\overline{\mathbb{C P}}^{2}-X=\left(\overline{\mathbb{C P}}^{2}-N_{i}\right) \cup\left(N_{i}-X\right)$ with $\overline{\mathbb{C P}}^{2}=\left(\overline{\mathbb{C P}}^{2}-\right.$ 
$\left.N_{i}\right) \cup N_{i}$ and using (c) above, we see the map $\pi_{1}\left(\overline{\mathbf{C P}}^{2}-X\right) \rightarrow \pi_{1}\left(\overline{\mathbf{C P}}^{2}\right)$ is an isomorphism. Hence $\overline{\mathrm{CP}}^{2}-X$ is simply connected.

By construction, $H_{*}\left(N_{i} ; \mathbb{Z}\right) \rightarrow H_{*}\left(\overline{\mathbf{C P}}^{2} ; \mathbb{Z}\right)$ is an isomorphism for $* \neq 1,4$ and $H_{1}\left(N_{i+1} ; \mathbb{Z}\right) \rightarrow H_{1}\left(N_{i} ; \mathbb{Z}\right)$ is zero by $(\mathrm{d})$. Since $\pi_{1}\left(T^{i}\right)$ is a free group on a finite number of generators, $H_{1}\left(N_{i} ; \mathbb{Z}\right)$ is torsion free by (a). Furthermore, the map $H_{*}\left(\overline{\mathbb{C P}}^{2} ; \mathbb{Z}\right) \rightarrow H_{*}\left(N_{i} ; \mathbb{Z}\right)$ is an isomorphism for $* \neq 1,4$ and the map $H^{1}\left(N_{i} ; \mathbb{Z}\right) \rightarrow H^{1}\left(N_{i+1} ; \mathbb{Z}\right)$ is trivial. From the definition of Čech cohomology and an easy limit argument, the map $H^{*}\left(\overline{\mathbb{C P}}^{2} ; \mathbb{Z}\right) \rightarrow \check{H}^{*}(X ; \mathbb{Z})$ is an isomorphism for $* \neq 4$. The Alexander dual of this map is the map $H_{4-*}\left(\overline{\mathbb{C P}}^{2} ; \mathbb{Z}\right) \rightarrow H_{4-*}\left(\overline{\mathbb{C P}}^{2}, \overline{\mathbb{C P}}^{2}-X ; \mathbb{Z}\right)$ [Gr, Chapter 27], so $H_{*}\left(\overline{\mathbf{C P}}^{2}-X ; \mathbb{Z}\right)=0$ for $*>0$.

1.0.5. Remarks. The Casson handles described in Theorem B are the first known examples of exotic Casson handles, i.e., Casson handles that do not contain a smoothly embedded standard 2-handle that trivialize their attaching circles (since any Casson handle smoothly embeds in the standard 2-handle exoticness is equivalent to being nondiffeomorphic to the standard open 2-handle). This family contains infinitely many nondiffeomorphic members: start with the simplest one, $C H_{1}$, with the minimal numbers given by the statement of Theorem B. The reimbedding algorithm produces another Casson handle, $\mathrm{CH}_{2}$, embedded in the first six levels of $\mathrm{CH}_{1}$. The new Casson handle, $\mathrm{CH}_{2}$, can be described by taking $y_{6}$ from Theorem B as "the input $x$ " in Theorem A. By repeating the construction one obtains a sequence of the Casson handles, $\left\{C H_{n}\right\}$ and $C H_{n+1} \subset C H_{n}$. A Casson handle $C H_{n}$ from the sequence can be used in the construction of an exotic $\mathbf{R}^{4}$ that is described in [B1]. The resulting exotic $\mathbf{R}^{4}$, denoted by $R_{n}$, depends only on (two copies of) $C H_{n}$, and the embeddings of Casson handles $\mathrm{CH}_{n+1} \subset \mathrm{CH}_{n}$ obtained by the reimbedding algorithm extend to embeddings of the associated exotic $\mathbb{R}^{4}$ 's, $R_{n+1} \subset \boldsymbol{R}_{n}$. Any compactly supported reimbedding in the opposite direction, $C H_{i} \subset C H_{j}, i<j$, will result in a reimbedding $R_{i} \subset R_{j} \subset R_{i}$ that is supported in a compact neighborhood of the involved Casson handles [B2]. That would produce a periodic end in the sense of Taubes [T], in the place that would contradict an argument from [DF] or [G5]. Details will appear in [B2].

The exotic $R^{4}$ 's constructed in [B1] are a different "kind" than the one constructed in the present work because they can be smoothly embedded in the standard open 4-ball and, therefore, in any smooth 4-dimensional manifold! It is easy to see that the above constructed exotic $\mathbf{R}^{4}\left(R=\overline{C P}^{2}-X\right)$ does not have this property. Any embedding of $R$ in $S^{4}$ could be used to form a closed manifold by identifying the ends of the complements of the embedded $R$ in $S^{4}$ and $M_{m}$. Such a manifold would have the intersection form equivalent to $E_{8} \oplus(m-9)\langle+1\rangle$, which is impossible by Donaldson [D].

\subsection{Ontline of the construction}

Remark 1.1.1. A family of "standard loops" for a kinky handle can be constructed from a certain family of loops on the core of the kinky handle. A kinky handle can be described as the result of self-plumbings of the standard 2-handle (or, equivalently, as a relative regular neighborhood of an immersed 
pair $\left(D^{2}, \partial\right)$ in the 4-ball, $\left(D^{4}, \partial\right)$, where the immersion has only double singular points with the preimages in the interior of the 2-disc). A construction of "standard loops" starts with a disjoint family of arcs in the core of the 2-handle (or in the domain of the immersion onto the core of the kinky handle). The end points of each of these arcs are a preimage of a double point, and for each double point such an arc is selected. The union of the images of these arcs in the core of the kinky handle is a disjoint family of loops, where each loop contains a unique self-intersection point of the core. A family of "standard loops" is obtained by isotoping the constructed loops from the core of the kinky handle to a disjoint family of loops in the boundary of the kinky handle. Without any loss of generality, the loops on the core of a given kinky handle will mean either the above-described loops on the core of the kinky handle or their isotopes in the boundary. Section 1.3.2 contains an outline of a variation of this construction in which the "loops on the core" are not required to be disjoint.

Our constructions will mainly take place in a collar neighborhood of the boundary inside the 0 -handle of the handlebody. We will use a diffeomorphism of the collar neighborhood and $S^{3} \times I$ with the properties that, under the diffeomorphism, $S^{3} \times\{0\}$ is identified with the boundary of the 0 -handle, and for every $t \in[0,1]$ the intersection of the "slice" $S^{3} \times\{t\}$ and $C_{1-6}^{0}$ up to an ambient isotopy looks the same as the link picture, i.e., $\left(S^{3} \times\{0\}\right) \cap C_{1-6}^{0}$. In other words, the collar neighborhood with the core of the tower scooped out is identified with $\left(S^{3}-\right.$ link $) \times I$. We will visualize the collar neighborhood as a vertical stack of 3-dimensional slices $S^{3} \times\{t\}$, where "down" will mean the direction in which "the collar coordinate" increases. The only changes allowed in going from slice to slice are ambient isotopies.

In our constructions we will often connect different 2-complexes by pipes that will run around the components of the 'input link' or around parts of the components, and we will typically have many parallel copies of the 2-complexes to connect. There is a degree of freedom in choosing the pipes, but we will always assume that the parallel copies of 2-complexes are connected by parallel pipes.

In section 1.6 we are going to find 1-stage capped gropes inside $T_{6}^{0}$ that are algebraically dual to the discs of the fifth level of the input tower. Then, the top stages of those capped gropes will be simultaneously contracted and the fifthlevel discs will be pushed off those gropes by Casson fingers (or 'finger moves'). These discs with the added Casson fingers are going to be $C_{5}^{1}$, the core of a new fifth level. A regular neighborhood of $C_{5}^{1}$ together with a small regular neighborhood of $C_{1-4}^{0}$ will be a new five-level Casson tower $T_{5}^{1}$ in $T_{6}^{0}$, with the core $C_{1-5}^{1}$, where $C_{1-4}^{0}=C_{1-4}^{1}$.

A new sixth level, $C_{6}^{1}$, will be constructed in section 1.7. Each "loop" of $C_{5}^{1}$ (that is, each of the "standard loops" of the kinky handles in the fifth level of $T_{5}^{1}$, obtained by an isotopy, as described in Remark 1.1.1), will be capped by an immersed disc. To construct such a disc, we will start with an embedded punctured disc bounded by the loop and a family of meridians of $C_{2-5}^{0}$. Then we will cap those meridians by immersed genus 0 cobordisms between them and some meridians of the fifth-level 1-handles, which in turn are capped by parallels of the sixth-level core discs. Because these kinky handles added on top of the $C_{5}^{1}$ are not disjoint, they form a 'fake sixth level'. 
The construction of $C_{6}^{1}$ continues in section 1.7.2. There, we construct a disjoint family of immersed spheres and 1-stage capped gropes that are dual to each of the parallels of the 6th level core discs included in the 'fake sixth level'. The sixth level is obtained in section 1.7 .3 by a variation of the singular Norman trick (described in section 1.5) that connects the immersed spheres with the kinky handles of the 'fake sixth level'. The net result of the singular Norman trick is that the intersections (and most of self-intersections) between the kinky handles of the 'fake sixth level' have been traded for the self-intersections on the added immersed spheres.

A seventh level is constructed by capping the loops on the sixth level by immersed discs, a 'fake seventh level' (sections 1.8.1 and 1.8.2). Then, the 1stage capped gropes constructed in 1.7.2 are contracted and sequentially pushed off each other. The resulting disjoint immersed spheres, together with the duals to the fifth level, are used in the singular Norman trick again (section 1.8.3) to trade the intersections on the 'fake seventh level' for new self-intersections.

1.2. Geometry of duals. We start with a description of the "elementary duals" which will be the building blocks of the reimbedding algorithm. A "dual" to a 1-handle will mean a surface dual to the missing core of the replacing scoopedout 2-handle. Each of the "elementary duals" consists of a parallel to the core of a 2-handle or a scooped-out 2-handle in the handlebody description of the input tower $T_{6}^{0}$. Such a "vertical part" is closed in a single three-dimensional slice by a "horizontal part", which is either a punctured surface or a surface with nonzero genus. Some of the punctures on a horizontal part will be connected by pipes with other elementary duals. In general, a connecting pipe will be linked with a 1- or 2-handle, and also with all elementary duals that contain a copy of the core of the same handle and that have their horizontal parts "below" the pipe. Consequently, the pipes can run in the 'vertical' direction only in the slices that do not contain those horizontal parts. We will build duals by using several 'operations' which are also described in this section.

Let $T_{k}$ be a symmetrized $k$-level Casson tower such that all discs of the $n$th level (for $n>1$ ) have $x_{n}$ positive and $x_{n}$ negative kinks.

1.2.1. Core discs. A specific description of core discs and their parallel copies is outlined next. Figure 3 represents several slices of a neighborhood of a positive kink on a kinky handle from $T_{k}$, i.e., a neighborhood of a piece of the 2-handle and one of the 1-handles from the standard handlebody description of a kinky handle. This kinky handle can be on any level of $T_{k}$ and we will describe for it a core disc and its parallels. On each of the consecutive figures in Figure 3, 'the collar coordinate $t$ ' is positive and higher than on the previous one. The coordinate $t$ of the slices in parts (a) and (b) of Figure 3 is in the interval $\left(0, \frac{7}{8}\right)$; for parts (c) and (d) $t$ is in the interval $\left(\frac{7}{8}, 1\right)$.

The core disc of the kinky handle contains the core of the included 2-handle. In other words, if the collar coordinate of part (a) of Figure 3 is set to be 0 , the visible piece of the core of the 2-handle would be its attaching circle. In part (a) itself, the visible piece of the core disc looks exactly as the attaching circle of the 2-handle and it is a piece of a doubled unknot which intersects the shaded area in two points. The shaded area is a piece of a parallel of the core disc and in the figure it is a disc with two punctures bounded by a longitude of the 2-handle. There is one more longitude in the figure. In slices "below", 
there is an ambient isotopy that slides one of the two components of the clasp through the other. The parameter of the isotopy is the collar coordinate. The Figure 3(c) shows the intersection point between the components of the clasp and part (d) is a picture of a slice "below", now meaning "after" the isotopy. In part (d), the visible piece of the core disc is unlinked from the scooped-out 2-handle so we can assume (by an ambient isotopy, if necessary) that the circle with the dot bounds a disc in this slice. Also, the remaining part of the core disc is visible as the shaded area. The boundary of the core disc is visible in part (d) and it is a meridian of the 1-handle on which the given kinky handle is attached.

Next, we will finish the description of a parallel copy (or, shortly, a copy) of the core disc whose piece is visible in (a) as the shaded area. This piece, which is entirely in the slice from part (a) will be called "the horizontal part" of the copy of a core disc. The longitude which bounds the horizontal part also has a parallel of the core of the 2-handled added to it. The boundary of the visible punctures is a Hopf link. This link is unlinked with the core disc but is linked with the other longitude in part (a). In slices immediately "below", the Hopf link is capped by a pair of intersecting discs. One of them is visible in part (b). The boundary of the parallel (denoted by $p$ in (a)) is also a meridian of the 1-handle on the level below. The other parallels of the core can be constructed by taking parallel copies of the horizontal parts in different slices. Those copies of the horizontal part are of course disjoint, but the pairs of discs added to the 'Hopf link-holes' intersect all other longitudes and, consequently, the other copies of the same core disc (see part (b)). The boundaries of the parallel copies, and the core disc itself, are all meridians to the same 1-handle on the level below, but each of these meridians is also linked with all copies of the longitude to the 2-handles that are pieces of the "vertical parts" that have their horizontal parts added in the slices "below" the one containing the meridian.

For the negative kinks, the pictures are the mirror images of those for the positive kinks and we will not discuss the obvious necessary changes.

An $n$th level core disc of the input tower is denoted generically by $d_{n}$. Because in our construction only the copies of core discs will be used and not the core discs themelves, we also refer to a copy as a $d_{n}$, or if there are more copies of the same core disc, as $d_{n}$ 's.

1.2.2. Characteristic tori. These are standard geometrically dual embedded surfaces used to obtain geometrically dual immersed spheres. The horizontal part of a characteristic torus is shown in Figure 4(b). The missing "vertical" part consist of an embedded disc parallel to the scooped-out 2-handle. The obtained torus has a natural trivialization of its tubular neighborhood, namely, the one extending the parallel of the scooped out handle on the horizontal part. An $n$th level characteristic torus is denoted by $\tau_{n}$.

Next, we fix some conventions. We assume that on each characteristic torus of the $n$th level there is a chosen symplectic basis of its first homology group. This basis is ordered by selecting the " + " and " - " element. Part (a) of Figure 4 represents the punctured caps, which are punctured discs bounded by the chosen symplectic base. Each of the punctures is connected by a pipe to a parallel of the curve denoted by $\mu$, which is a meridian of the 2 -handle visible in the figure. 
Furthermore, we assume that for each $n$th level kinky handle there is a path in the link picture of the input tower which connects the meridian $\mu$ with boundaries of all punctures of the punctured caps for the characteristic tori in the kinky handle. This path is in the boundary of a tubular neighborhood of the attaching circle of the 2-handle (in the given kinky handle) and its defining property is that, if its end on $\mu$ were connected with any puncture by an arc in the picture which is above the link, the piece of the path together with this arc should be (homologically) unlinked with this attaching circle (see Figure 5). We will translate this picture into the interior of the 0 -handle of the handlebody decomposition of $T_{k}$ and refer to the obtained path as a "guiding path". A "guiding path" has a double role. First, it will be used as a guide for the pipes connecting the $\mu$ with a puncture on a cap of a characteristic torus, by requiring that each pipe be centered at the attaching circle and that the appropriate piece of the guiding path lies on the pipe. Later, Casson fingers that run over the pipe will have the pieces of the guiding paths as their centers.

1.2.3. Core surfaces. For each integer $n$ such that $1 \leq n \leq k$ and for each core disc on the $n$th level, there is an embedded surface with the same boundary and the same vertical part as a copy of the core disc. We will assemble each of these "core surfaces" from several pieces. Figure 6 represents a piece of the $n$th level of tower $T_{k}$ or more precisely, an intersection of a regular neighborhood of a $n$ th-level core disc (= kinky handle) and a three-dimensional slice of the 0 -handle. The shaded areas in the figure are pieces of the horizontal part of a core surface. The shaded punctured sphere that is visible in part (a) of the figure has $\left(2 x_{n}+2\right)$-many holes. In the figure only four of them are visible: one is the circle bounding the shaded area and this circle is a preferred longitude of the attaching sphere of the 2-handle. Two more circles are the places where the attaching spheres of the 1-handles pierce the shaded area. Because on a core disc in the $n$th level there are actually $2 x_{n}$ kinks and therefore that many 1-handles, these two holes in the figure represent two of the $2 x_{n}$ similar holes. The fourth hole in the figure is the puncture due to scooping out the 1-handle from the $(n-1)$ st level (the 1-handle on which the 2-handle from the figure is attached) and is denoted by $p$ in Figure 6(a).

We cap the longitude (the first hole) by a parallel copy of the core of the 2handle. In Figure 6(b) there is a punctured torus associated to each kink of the $n$ th-level core disc visible in the figure (again only two of them are visible). Each of the next $2 x_{n}$ holes of the shaded area is capped by the associate punctured torus from part (b) of Figure 6. The last hole $p$ is the boundary of the surface.

The framing of the visible 2-handle is extended to the whole surface in the obvious way. The induced framing on the boundary $p$ is the 0 -framing with the respect to the slice depicted by Figure 6. As in the case of the characteristic tori, we again assume that for each of the $2 x_{n}$ added punctured tori (see Figure 6(b)) there is a fixed chosen ordered symplectic basis for its fundamental group. This specified basis should be represented by a wedge of two circles.

The $n$ th-level core surfaces are denoted by $F_{n}$.

We can produce arbitrarily many disjoint parallel copies of the above constructed surface by placing their horizontal parts in different three-dimensional slices. The only thing to check is that for each copy of the surface constructed above, the parallel copies of the core of the 2-handle that end on the longitudes 
in the slices "below", are not intersecting the surface. In the slice of Figure 6, these parallel copies of the core of the 2-handle are visible as additional copies of the preferred longitude, i.e., the unknots unlinked with the visible piece of the core of the 2-handle. It can be easily arranged for them to be between the 2-handle and the horizontal part in Figure 6(a), so they will not intersect the surface. However, note that a core disc with its horizontal part "above" the slice from Figure 6 intersects the core surface, because in the slice containing the horizontal part of a core disc, the visible piece of the core surface is a longitude linked with the 'Hopf link holes' (Figure 3(b)).

For each of these parallel copies of the surface we assume the parallel choice of the symplectic basis for the fundamental groups of the added tori.

1.2.4. Punctured geometric duals. We will construct a punctured geometrically dual surface for each 2-handle of the $n$th level of $T_{k}$. Figure 7(a) represents an intersection of a three-dimensional slice of the interior of the 0-handle and a neighborhood of the 1-handle from the $(n-1)$ st level on which the 2-handle from the $n$th level is attached. A piece of the 2-handle is visible as an arc. Also, there is a piece of a 2 -handle from the $(n-1)$ st level which is visible in the figure.

In Figure 7(a) there is a shaded area which is a sphere with five holes near the 1-handle from the $(n-1)$ st level. The circle denoted by $\mu$ is the boundary of a disk transversal to the $n$ th-level 2 -handle. The holes in the shaded area are the four meridians of the attaching sphere of the 1-handle and a preferred longitude of this attaching sphere. Figure $7(b)$ is ambiently isotopic to Figure 7(a). It is an application of the introduction of a kink, as described by Figure 1. The shaded area in this picture is going to be the horizontal part of the dual. It has both a negative and a positive clasp, but just one of them is linked with the core. Again, we have five holes as Figure 7(a). The longitude is capped by a parallel copy of the core of the scooped-out disk replacing the 1-handle, and the four meridian holes form two Hopf links. As indicated in Figure 7(c), the four holes in the shaded area that are meridians to the 1-handle are connected by pipes with the boundaries (denoted by $p$ in Figure 6 ) of the different copies of the $n$ th-level punctured surface from Figure 6. Each of these four copies of $p$ is in a different slice. We call this surface a punctured dual and denote it by $P_{i}$, where $i$ stands for the level in which the punctured dual is situated. A copy of $P_{n-1}$ is dual to a core disc in the $n$th level and is disjoint from the rest of the core of the tower $T_{k}$. This intersection is visible in parts (a) and (b) of Figure 7 as the center of the disc denoted by $\mu$ in these figures.

We frame this surface by starting with the framing for the parallel of the core of the 1-handle (the scooped-out 2-handle) which is added to the preferred longitude "bounding" the horizontal part. Then, the framing is extended to the horizontal part in the obvious way, so that the meridian holes obtain the 0 -framing in the slice. The pipes are also framed so that the induced framings of their other ends are the 0 -framings (with respect to the slices containing the other ends). If 2-handles were attached to the holes of this framed punctured surface, the result would be diffeomorphic to $S^{2} \times D^{2}$.

Copies of punctured dual $P_{n}$ 's on the $n$th level will be used to cap the $(n+1)$ st-level meridians of the 2-handles. To produce arbitrary (finitely) many copies of a punctured dual, two methods will be used. 
(a) To cap a collection of parallel meridians of the same $(n+1)$ st-level 2handle that are linked with the same collection of the $(n+1)$ st-level core disks or surfaces (i.e., which are homotopic in the complement of the core), we construct the horizontal parts of all parallel copies of a $P_{n}$ in a single slice. In Figure 7 (d), pieces of two such parallel horizontal parts are visible. Adding the pipes to the meridian holes in the slices "below" introduces intersections and selfintersections between the resulting surfaces. The reason for that is that all meridian holes form two Hopf links whose components are replaced by families of their unlinked parallels. Consequently, each parallel of a punctured dual together with the added pipes has a ' + ' and a '-' kink. Also, each pair of parallels has four intersection points.

(b) We will make disjoint from $P_{n}$ those punctured duals parallel to the $P_{n}$ that cap meridians of the $(n+1)$ st-level 2-handles which are linked with a collection of core surfaces different from the boundary of the $P_{n}$. In the slice that contains the shaded area of the $P_{n}$, these parallel surfaces are visible as unlinked longitudes to the 1-handle (see Figure 7(d)) which do not intersect the horizontal part, but instead pass through its meridian holes. These holes are piped to the added surfaces in the complement of these longitudes (i.e., a longitude passes unaffected in the "vertical" direction throughout $P_{n}$ and the pipes added to it).

1.2.5. Operations on duals. We will describe several operations which produce new dual surfaces from the "elementary duals".

The chosen symplectic basis for each punctured torus added to a core surface represents the surgery data necessary to surger the punctured tori to a disc. The basis is described by Figure 6(b) for a core surface and by Figure 4 for a characteristic torus. This data is used in "surgery on the middle level of an even-dimensional manifold", which can be performed by either surgering out the "Lagrangian", i.e., the first circle in the symplectic pair, or by scooping out from the surface a regular neighborhood of the wedge of two circles representing the whole symplectic basis and attaching on the resulting boundary component (which is a circle) a disc. In the latter case the whole surgery kernel is cut out and a special case of this operation is called the "contraction" of a 1-stage capped grope in [FQ], where it is carefully explained. The version of it that we will use is the following operation.

$\mathcal{O}_{1}$ (the double surgery). In a core surface on the $n$th level, each of the added punctured tori (Figure 6(b)) is replaced by: (a) an immersed disc which is a contracted 1-stage genus 1 capped grope or (b) by a higher genus surface.

(a) The replaced capped grope consists of a punctured torus with both symplectic generators piped to copies of core discs in the $(n+1)$ st-level. The body of the grope is a parallel copy of the replaced punctured torus. The caps consist of the two punctured discs bounded by the chosen wedge representing the symplectic basis. Each of these discs has a puncture where the 1-handle is scooped out. Then, each puncture is piped to a copy of a core disc one level up, as sketched in Figure 8. Each pipe ends in a different slice on a copy of the core disc. The resulting capped grope has intersecting caps and the caps also intersect its parallels. To contract this capped grope we have to double the caps, meaning that we have to construct an additional copy of each cap (see Figure 
13). The two additional copies of the caps can be constructed so that all four caps are in different slices. The result of the contraction is an immersed disc with the same boundary as the punctured torus.

(b) In the given core surface on the $n$th level, replace the added punctured tori by the surfaces with the same boundaries and of the following type. From the punctured torus cut out a wedge of circles that represents the symplectic basis, as in the operation $\theta_{1}$ (a). Then to the new boundary add four copies of the $(n+1)$ st-level core surface (as in Figure 13) to obtain a new surface which is a 2-stage grope with its bottom stage contracted.

In case (b), this operation applied to several parallel copies of a core surface yields disjoint surfaces. In the case (a), the result is a family of immersed discs that intersect each other. The intersections come from the intersections of parallel caps of different capped gropes. However, the parallel bodies are assumed to be disjoint. If we apply this operation on core surfaces from different $n$th level kinky handles, then the resulting immersed discs will be disjoint because the caps will be parallels of different $(n+1)$ st-level core discs.

The next operation is a singular version of the standard surgery of a torus.

$\theta_{2}$ (the single surgery). Instead of producing a capped grope, this operation performs a surgery on the first element of a symplectic basis. Each of the added punctured tori is replaced by one of the following two surfaces.

(a) The following immersed disc: An annulus centered at the circle representing the ' +' element in the symplectic basis is removed from the punctured torus. Each of the two new boundary components is connected by a pipe around the 1-handle with a copy of the core disc in the $(n+1)$ st-level (see Figure 8). The two pipes used are parallel except near one end, where one of them is pushed into another slice.

(b) Surfaces with the same boundaries and of the following type: From the punctured torus cut out the annulus centered at the circle representing the ' $t$ ' element in the symplectic basis and add two parallel pipes (as in the operation $\left.\theta_{2}(a)\right)$. The ends of the pipes are two parallels of $\omega$, each in a different slice. Then add to each of them a copy of the core surface bounding it; each with its horizontal part in the respective slice.

If this operation is applied to several core surfaces in the $n$th level, we will have the same conclusions about the intersections between the resulting immersed discs or surfaces, as we did for the operation $\theta_{1}$.

The following two operations use a characteristic torus from the $n$th level instead of a core surface. See Figure 4.

$\theta_{3}$ (the double surgery). Use "the double surgery" on an $n$ th-level characteristic torus by cutting out the entire symplectic base and connecting the punctured disc with a surface on the $(n-1)$ st level. Connecting pipes follow the guiding paths (see Figure 5$)$ until the $(n-1)$ st-level 1-handle. The added $(n-1)$ st-level surfaces are:

(a) four copies of the punctured dual $P_{n-1}$ on the 1-handle, or

(b) four copies of the characteristic torus $\tau_{n-1}$ on that 1-handle.

Analogous to the operation $\theta_{2}$ is:

$\theta_{4}$ (the single surgery). The '+' element of the symplectic base of an $n$ thlevel characteristic torus is surgered by two copies of:

(a) the punctured dual $P_{n-1}$ on the 1-handle below, or 
(b) the characteristic torus $\tau_{n-1}$ on that 1-handle.

There is one more operation that we are going to use and it has been already described in section 1.2.4:

O5 A copy of a $n$ th-level punctured dual $P_{n}$ is connected by pipes (as described in 1.2.4) with four copies of either the core surface or the core disc on the attached $(n+1)$ st-level 2-handle.

1.3. Loops. In the construction of the embedded Casson tower we will cap in each new level a standard family of loops. Typical pieces of the loops involved in the construction are described in this section.

1.3.1. Conventions. The loops on the fifth and the sixth levels will consist of pieces which are in separate slices and which are connected by pairs of parallel arcs. Also, each of these pieces will be unknoted in the respective slice and unlinked from its parallels. However, these pieces of the loops will be linked with the core in the respective slices, meaning that the obvious discs they bound will have punctures in the complement of the core. The punctured discs that are bounded by pieces of a single loop, will be connected by ribbons.

Figure 9(a) describes how the pieces of the parallel loops can be capped by disjoint punctured discs. Namely, by changing the collar coordinates, we can embed disjoint annuli bounded by parallel pieces of the loops. Then, for each loop, we choose a separate slice in which the loop is capped in the complement of the annuli for its parallels. Note that this is always possible as long as the parallel pieces are unlinked unknots in the selected slice.

We can compress the slices that contain the annuli of all pieces of the loop to a single slice. Equivalently, provided that the loop satisfies a modest additional condition that it does not contain pieces that are vertical with respect to slices, there is a well-defined isotopy which "slides" the different pieces into a designated slice. This isotopy is defined in regular neighborhoods of the annuli and can be trivially extended outside this neighborhoods to an ambient isotopy. We will call this isotopy a projection.

Besides allowing us to construct a loop in one or several simple pictures, this isotopy will be used to frame the loops in the following way: The "projected loop" in a single slice $\left(=S^{3}\right)$ can be framed by selecting its longitude. Using the inverse of the projection we obtain a "longitude" for the original loop. This new longitude determines a framing of the loop, i.e., a trivialization of a solid torus with the loop as a core. By this convention the "0-framing" for a loop is well defined.

1.3.2. Intersecting standard loops. Let us consider the following situation. Assume that we have an immersed disk with equal numbers of positive and negative kinks. The fundamental group of the (image of the) immersed disk is a free group with the number of free generators equal to the total number of kinks. Furthermore, assume that there is an ordered set of pairs of free generators for the fundamental group of the immersed disks, consisting of embedded loops which are in the general position and each of them passes through exactly one self-intersection point. Each pair is assumed to consist of a free generator for a negative kink and one for a positive kink. Also, loops of each pair are either disjoint or they intersect in one point. We will suppress the base point in this discussion. 
Lemma 1.3.2. Suppose that there is an ordering of the pairs of free generators for the fundamental group of an immersed disk as described above. Also, suppose that for each pair both of the two representing loops of the pair intersect two loops from any previous pair in the same number of points and that these intersections can be paired such that each part of the loop between a pair of intersections does not intersect any other loop. Then, the given set of free generators can be framed to form a "standard family", i.e., the regular neighborhood of the immersed disk is a kinky handle and after the loops are lifted to the boundary of the kinky handle and 2-handles are added on them, the resulting manifold is a 2-handle with the same attaching circle.

Before we prove the lemma let us recall "the standard picture", when we have a disjoint standard family of loops on the surface.

If there is only one kink on an immersed surface, then the kink can be capped in the following way. The two preimages of the double point are connected by an embedded arc on the surface. Under the immersion, the arc is an embedded loop passing through the kink. A picture of a regular neighborhood of the loop inside the kinky handle is represented in Figure 10(a). In this figure, the piece of the surface around the loop is visible as a disc with two holes which form a Hopf link, together with two intersecting discs bounded by this Hopf link. Again the neighborhood $\left(\cong S^{1} \times D^{3}\right)$ is "sliced" in a 1 parameter family of three-dimensional objects, in this case, in a family of solid tori. After removing an open neighborhood of the loop from the visible piece of the surface, the remaining piece of the surface has a hole which is a doubled unknot. In other words, the capping pair of the discs for the Hopf link is scooped out and the 2 holes are connected by removing a band. This piece of the surface is now in a single "slice", as shown in the topmost slice of Figure 10(b).

The "slicing" of a neighborhood of a loop determines a framing for the loop, meaning that the loop is pushed into the boundary of the kinky handle by projecting it into a "slice" in the boundary. There the loop is an unknot with the 0 -framing coming from considering this slice (= solid torus) to be imbedded in a 3-ball. Using this framing, a 2-handle can be added. The neighborhood of the loop, with the loop itself scooped out from the surface, together with the 2handle is a 4-ball and the hole in the surface is capped as in Figure 10(b), that is by two copies of the core of the 2-handle which are connected by a once-twisted band.

Remark. The framing of the resulting embedded surface differs from the initial one by \pm 2 , due to the change in the (geometric) number of self-intersection points by one. This construction does not change the homology class of the surface.

In the general situation, when there is more than one kink, we can find a disjoint collection of arcs connecting the preimages of the double points and locally we have the same picture (see Remark 1.1.1). If the number of positive kinks is equal to the number of negative kinks, the resulting framing of the surface will be unchanged.

Proof of the lemma. In the situation of the lemma, we do not necessarily have disjoint loops. If two loops on the immersed surface intersect each other, we can still cap one of them as before by adding a 2-handle. Now the other loop 
is not on the surface any more, the part which was intersecting the scooped out open neighborhood of the first loop is missing. However, the missing part can be replaced by an arc going over the two discs added on the first loop and the twisted band that connects them. When the second loop is scooped out, the new hole will be the once-twisted double of the unknot (relative to the "slicing" as above); the twist comes from the loop following the twisted band and has the opposite sign from the kink removed by capping the first loop. Furthermore any other loop intersecting the first loop has to follow the band and consequently will end up being linked with the second loop.

This situation is described by Figure 11. In part (a) of the figure, the added pieces to the boundary of the complement of the two loops in the surface, are drawn with the bold line. The resulting change to the second hole is shown in part (b). There the part between the vertical gap is a piece of the second loop that goes over the doubled core of the 2-handle and the added band. Also, in the figure, there is one more loop shown that now twists around the second loop.

In the situation of the lemma, each I-pair of loops intersects the previous pairs in an equal number of points, meaning that, after the above-described capping of the previous loops, the loops from the pair will get the same number of positive and negative twists. Also, their linking number will remain the same. If the loops in the pair were disjoint, the described procedure would produce untwisted and unlinked holes that can be capped as described above.

In the case that the two loops from a pair intersect each other in a point, the above procedure will not introduce any new intersections or link the two loops. The framing for the first loop is obtained as before, but the framing of the second loop is set to be the sign of the kink through which the first loop passes (i.e., by \pm 1 ).

Figure 12(a) shows a piece of the boundary of a hole produced by scooping out an intersecting pair of loops. Also visible are pieces of two of the loops (the bold curves) to be capped after the given pair is capped. In the case shown in the figure, the first loop has a positive kink and the second a negative. Therefore, the framing for the second loop is set to be " 1 ", and, after the second loop is scooped out, the resulting hole is a positively twisted double. Part (b) of the same figure is the result of an isotopy that moves the remaining loops that intersect the pair in such a way that the resulting loops do not intersect the second loop from the pair. This isotopy moves only the arcs that connect pairs of intersection points and which are disjoint from all loops but the given pair. Capping off the first loop results in part (c). There, the "missing" piece of the second loop was replaced by a (negatively) twisted band as before (see Figure 11). All pieces of the remaining loops that intersect the pair are now twisted and linked with the new second loop, but an isotopy that undoes the two \pm -twists untwists the band and unlinks the remaining loops. The second loop can be capped now so that the remaining loops are disjoint from the capping disc.

1.4. Loops on duals. All self-intersections on the new sixth level will be on a disjoint family of duals. Those duals will contain several levels of contracted capped gropes and kinky handles capping the loops for their self-intersections will form the seventh level. The included gropes will be build from characteristic tori or from core surfaces using the operations $\theta_{3}$ and $\theta_{1}$.

The reason for using double surgery on tori (instead of the more usual ' + 
surgery') is that the intersections between the resulting contracted capped gropes can be traded for self-intersections. This procedure uses the Casson fingers and will be described next.

\subsubsection{Casson fingers on contracted capped gropes. The general situation that} we have is described by Figure 13. This is a generic picture of a contracted capped punctured torus (denoted by $c G$ in the figure) which is a piece of a characteristic torus or a core surface and is altered by the operation $\theta_{1}$ or $\theta_{3}$. In the figure we can see pieces of an intersection of the cores of three handles (denoted by $A, B$ and $C$ ) and a three-dimensional slice. Also, a pair of intersecting Casson fingers is visible in the figure as two pairs of parallel bold arcs ending on a Hopf link. We are assuming that there are arbitrarily many parallels of each of the two visible fingers, so one can view the bold ares as being cables of parallel arcs. The two doubled caps of $c G$ consist of the four oncepunctured discs whose holes are bounded by the meridians of $B$ and which are connected by pipes with the four copies of a surface bounded by $C$. The four pipes used are concentric to pieces of $B$. Their ends are numerated and a selected orientation of the body induces orientations of the four parallels of C.

The pieces of the Casson fingers invisible in Figure 13 are described by Figure 14. In Figure 14, there are several intersections of fingers and three-dimensional slices. Two representatives of each family of the fingers are drawn. The slice (7) is the one from Figure 13, and the slices (1)-(6) are "above", showing the half of the intersections between the fingers. The slice (5) differs from the slice (7) by a regular homotopy of a clasp that slides its two components through each other. The induced intersection point is visible in the slice (6). Similarly, the remaining visible clasp is "undone" between the slices (4) and (2), with an intersection visible in the slice (3). After all arcs are unlinked (slice (2)), the two families of arcs bound disjoint half-open discs (slice (1)). The picture is repeated "below" the slice (7), in the "down" direction, until the slice (8).

Figure 15 represents a detail of Figure 13 in which the two families of intersecting Casson fingers are also represented only by a member of each.

Two intersecting families of Casson fingers can be added to any surface that intersects the caps of the contracted capped grope from Figure 13. Note that for each intersection between a surface and a cap of (an uncontracted) capped grope, there are two intersection points between the surface and the doubled cap. The above-mentioned Casson fingers are used to remove such pairs of intersection points. Figure 16 describes an example of a Casson finger added to a surface $s$ to remove its pair of intersections with two parallel "elementary duals" that are pieces of a doubled cap and that are denoted in the figure by $c_{1}$ and $c_{2}$. The upper part of Figure 16 represents a bicollared neighborhood of the horizontal part of $s$ inside the 0 -handle. Six characteristic three-dimensional slices are shown below, giving a description of the Casson finger added to $s$. The finger is linked with a doubled cap and continues over it until the body of the grope where it ends as described in Figure 13 or Figure 15.

Let us suppose that there is a family of contracted capped gropes, as the one in Figure 13, such that each has its body in a separate three-dimensional slice and that their caps are formed from parallel "elementary duals". By adding Casson fingers, we can make the family of contracted capped gropes disjoint. 
First, the family is ordered and then all intersections between the first and all remaining gropes are removed by adding Casson fingers to the caps of all but the first of the contracted capped gropes. Now, the first grope is unchanged, but it is disjoint from all the other gropes. The other gropes have new intersections and self-intersections, due to the intersection of the added Casson fingers. Next, the gropes from the family are made disjoint from the second grope. Again, the first grope is unchanged, the second one has only fingers avoiding the first one and the others also have Casson fingers that avoid the second grope. After repeating the process as many times as there are gropes in the family, we have a disjoint family of contracted capped gropes.

Remark. Only one grope from the family is unchanged (the first one). All others have progressively more self-intersections, so the "symmetry" of our construction will be lost, unless we add more kinks to all but the last grope in the family.

\subsubsection{Loops for Casson fingers. Let $K$ be a kinky handle and let $C$ denote} its core. Suppose there is a pair of Casson fingers with a \pm pair of intersections that is added to $C$ inside the kinky handle $K$. Denote by $C^{\prime}$ the resulting self-intersecting disc and by $K^{\prime}$ a regular neighborhood of $C^{\prime}$ in $K$, i.e., a kinky handle in $K$ with the core $C^{\prime}$.

A link calculus picture of $K^{\prime}$ is Figure 17. In the figure, the dashed circles denoted by $a$ and $w$ show two possible extensions of the standard basis of $\pi_{1}(K)$ (i.e., a basis consisting of standard loops) to a basis of $\pi_{1}\left(K^{\prime}\right)$. If we add a 2 -handle to the circle $w$ with its 0 -framing the fingers are "undone", i.e., in the union of the $K^{\prime}$ and the 2-handle there is an obvious regular homotopy of $C^{\prime}$ to $C$. However, adding $w$ to the standard basis of $\pi_{1}(K)$ is not an extension to a standard basis of $\pi_{1}\left(K^{\prime}\right)$. The other possibility is to add two 2 -handles, one to $a$ and another to $a \pm w$, both equipped with the 0 -framing. It is easy to see that this gives a standard basis of $\pi_{1}\left(K^{\prime}\right):$ simply slide the inner clasp over (the 2-handle with 0-framing) $a$ and the remaining one over $a \pm w$.

Because our construction of the new Casson tower does not use the link calculus, we are going to give another description of the $C^{\prime}$. We assume that $K$ is a regular neighborhood of its core in a 4-ball. Furthermore, as for the input tower, we assume that $K$ is in a bicollared neighborhood of a 3-ball inside the 4 ball. In Figure 18(a) we can see the following piece of $C^{\prime}:$ the finger to the left is fully visible, and of the other one we can see its attaching circle and two parallel arcs which pierce the first finger and are connected "inside" the first finger. Also in the figure there is a neighborhood in $C$ of the attaching circles of the fingers and of an arc which connects these circles. This picture is in the 3-ball which was assumed to have the above-mentioned bicollared neighborhood in a 4-ball. The remaining pieces of the right-hand finger are two discs which are assumed to be "above" and "below" in the bicollared neighborhood. This arrangement can be easily obtained by an isotopy changing the "collar coordinates" of the fingers and the connecting arc on the $C$. Although this part of $C^{\prime}$ (an immersed disc) was drawn in a 4-ball, the picture we are looking at consists only of a regular neighborhood of the described immersed disc in the 4-ball, which we temporarily denote by $N$.

The loops we are going to use are a parallel of the boundary of $a$ and a parallel of the boundary of $a \pm w$, which are drawn as the bold dashed closed curves in Figure 18(a). In this picture of the 3-ball, the boundaries of $a$ and 
$a \pm w$ have the 0 -framing. (Note that in this 3-ball these two curves are isotopic, but that is only the result of the circle $w$ being trivial in the 3-ball.) Each of the two loops is defined as the union of a pair of arcs on the fingers and a parallel of the arc that connects the fingers. These arcs start from an intersection point of the fingers and together with the parallel of the connecting arc are in the boundary of $K^{\prime}$.

Part (b) of the figure is a diffeomorphic picture of $N$ where the left finger was isotoped back to $C$ (compare with [FQ, section 3.1]). The other finger is still visible as a curve and still pierces the rest of the disc in two points. The discs bounding $a$ and $w$ are fully visible.

We show that the addition of two 2-handles to $N$ will produce a 2-handle with the same boundary. First we split $N$ into two symmetric parts. In Figure 18(a) one can see a "dot-dash" curve on the left finger. This curve is extended to the left until the boundary of the neighborhood of the connecting arc on $C$. To the right it is the connecting arc itself, until the right-hand finger. After the finger, the curve connects the attaching circle of the finger with the boundary of the neighborhood of connecting arc on the right-hand side of the figure. We assume that on the fingers this curve cuts each finger into two discs, each containing one of the intersection points. We split the surface by cutting it across this curve and extend it to a decomposition of its neighborhood $N$ into two parts. Part (c) of Figure 18 shows the "upper half" of $N$ after it is split across the described curve. This is the standard picture of a neighborhood of a disc with one positive kink, and after adding a 2-handle to the loop that was drawn bold in Figure 18(a), the resulting manifold is a 4-ball. The closed curve in Figure 18(c) is an unknot and is a slice knot in the 4-ball. The "lower half" of $N$ differs in having a negative kink. After adding a 2-handle to it, we glue the two slice discs together by identifying the copies of the splitting curve. The resulting surface is shown in (d) and it is a disc embedded in $N$ with the same boundary as the immersed disc described by part (a) of the figure. So we again see that adding two 2-handles to the described loops gives a kinky handle diffeomorphic to $K$.

We proceed to a more general case when $C^{\prime}$ is obtained from $C$ by adding arbitrarily many Casson fingers. We suppose that these fingers have all integral intersection numbers equal to 0 , meaning that the intersection points between the fingers can be grouped in \pm pairs. The loops which we are going to use are the same as before. From each intersection point between two fingers, we run an arc over each finger. The remaining end points of the arcs are connected as before by a "connecting arc" on $C$. Then the obtained loop is lifted to the boundary of $K^{\prime}$. The "connecting arcs" used for all intersections of any two fingers are the same, but we are taking disjoint lifts of these loops.

To see that these loops are an extension to a standard base of $K^{\prime}$, we use the previous argument. Starting with any intersecting pair of Casson fingers we cap all its loops by 2-handles. The argument above shows that the new kinky handle we obtained has lost those kinks. Still, the new kinky handle has retained the intersections that were between the other fingers and the removed pair. Each pair of these intersections between a remaining finger and the new core disc now looks like part (b) of Figure 18. Using the isotopy that sends part (b) to part (a) restores the removed two Casson fingers but now they are mutually disjoint. In this manner we remove all intersection between the fingers. The resulting 
surface is another core disc for the original (= without fingers) kinky handle $K$.

In all cases that we will consider, self-intersections and intersections of caps will be only on the second-level punctured duals, the fifth-level core discs and on the Casson fingers added to the duals. Because of the "symmetrization" of all core discs and the addition of an extra kink to each punctured dual, all capped gropes considered do have an equal number of positive and negative kinks. For the fingers from Figure 13 a visible piece of the loop is described in Figure 19, where this piece, together with a line segment (denoted by $p$ in the figure), bounds a punctured disc (shaded in the figure). A neighborhood of this annulus is framed so that its boundary is two unknots with 0-framing: the piece of the loop closed by $p$ and a meridian of $A$. This piece of the loop is unlinked with the Casson fingers and outside of the picture it follows the two fingers up to their beginning. Then, as explained above, the loop is closed by an arc on the surface to which the two fingers were added.

\subsubsection{Loops on caps. Beside the intersections introduced by adding Casson} fingers, a contracted capped grope has intersections and self-intersections coming from the intersections of the core discs and the punctured duals used to construct the caps. The intersections and self-intersections of the caps are not visible in Figure 13 or Figure 15, but some of their loops should enter the figure because the caps are parallel surfaces connected on the body of the grope. The pieces of the punctured discs will be parallels of the "model" pieces visible in Figure 20. There one can see a once-punctured disc for each of these loops and a ribbon connected to the punctured disc ending on an arc denoted by $p$ in parts (a)-(c) of Figure 20. The puncture for a loop in (a) is a meridian of $A$ and that for a loop from (b) or (c) is a meridian of $B$. The way these pieces fit together on a body of a grope is shown in Figure 21. This is the same part of a contracted grope as in Figure 15, with the difference being that the pair of Casson fingers is drawn with dashed lines. The arcs on the body of the grope, drawn with bold curves, are pieces of the "model" loops. The rest of the "model" loops are indicated in Figure 21 by the arrows, and in Figure 20 each of them is drawn separately.

Loops for different intersections are obtained by taking parallels of the arc from Figure 21. It is obvious that in some cases it is impossible to avoid intersections between these loops. However, we can use Lemma 1.3.2 to obtain a standard family of loops on a contracted capped grope. Before the addition of the Casson fingers, all caps had equally many positive and negative selfintersections. The intersections between Casson fingers are always in \pm pairs, so adding Casson fingers does not disturb this symmetry. As a consequence, we can group the self-intersections in \pm pairs. Figure 22 shows a piece of the "horizontal part" of either a core disc or a punctured dual (the shaded area) near the boundary of this elementary dual. The visible pieces of the loops for the kinks on the surface are illustrated by three pairs of \pm curves. Their ordering is the one from Lemma 1.3.2, i.e., the order in which the pairs are to be capped by kinky handles. The visible pieces of the loops end on the boundary of the elementary dual, which is also an end of a pipe leading to the body of a grope. On the pipe and afterwards, the loops are easily arranged to be disjoint up to the pieces from Figure 21. Then, using Figure 13 and Figure 21, one can see that 
a \pm pair of loops with intersections between caps with the same orientations remains disjoint. A pair of loops on caps with different orientations has an intersection point on the surface from Figure 21. The arcs in Figure 21 and their translates (in general position) now represent pieces of \pm pairs of loops. Here each loop intersects the other pairs in \pm pairs of points. Consequently, the intersections on the body of the grope do not put any requirement on the ordering of the pairs. The ordering of the loops is then generated by requiring that the loops on the added Casson fingers come first (with an arbitrary ordering) and then ordering the loops by the procedure described by Figure 22.

Hence, all the conditions of Lemma 1.3.2 are satisfied, provided of course, that the chosen family of loops is a set of free generators of the fundamental group of the given contracted capped grope.

1.5. Singular Norman trick. We are now going to outline briefly a particular variant of the (singular) Norman trick which will be used throughout the construction of a new Casson tower. A more general and more complete description can be found in [FQ, section 1.9].

The Norman trick itself is to connect a kink of a surface with an intersection point of the surface and a geometrically dual sphere, by an arc over the surface, and then to take a tube around the arc with one end circle on the surface and the other on the dual sphere. Then, the interiors of the discs bounded by the end circles of the tube are deleted, one from the surface and the other from the dual sphere. The resulting union of the surface-the dual and the tube without those two open discs-is nothing but a connected sum of the surface and its dual. But because the kink was in the interior of a disc thrown out in the construction, the resulting connected sum has one kink less than the original surface. Consequently, the Norman trick is just a carefully performed connected sum of a surface and its dual. If the dual sphere was just immersed, the resulting surface would get all the kinks of the dual instead of one of its own, but this is sometimes an acceptable price to pay for the removal of an unwanted kink. In the case that the geometric dual is an immersed sphere, the construction is called the singular Norman trick.

We will use the singular Norman trick to 'remove' (in the above sense) intersections and self-intersections of a family of parallel surfaces. Figure 23 was drawn according to the conventions used in [FQ]. Briefly, it represents a threedimensional "slice" or the "level" in which some parts of surfaces are visible and from others we can see only arcs. In Figure 23(a) we can see pieces of three parallels of a part near a single kink of an "original" surface, and also the place where the parallels intersect the dual (which is drawn as an embedded sphere). The only pieces visible of each of the parallels to the surface are a square and a line. The whole picture (a) is a "slice" in which one of the two intersecting discs forming a kink is visible and for the second one we can see only a line segment. In part (b) of the figure, one can see the result of the Norman trick which eliminated the intersections depicted on part (a). The intersections coming from the other kinks of the "original" surface are piped through the pipes in the (b), and if there are $m$ parallels of a surface with $k$ kinks, $(m \cdot k)$ dual spheres are needed for the Norman trick.

Remark 1.5. From Figure 23, it is also obvious that the sphere in figure (a) is geometrically dual to each of the parallels separately, but if several parallels are 
part of the same surface, strictly speaking, the sphere is not 'dual' to the surface, but the "trick" from figure (b) still works.

1.6. Construction of the fifth level and its duals. This step is the "Improved three-stage Reimbedding Theorem" [GS, (3.3)]. A new fifth level and its geometrically dual spheres are produced by contracting geometrically dual 1-stage capped gropes.

1.6.1. $\quad C_{5}^{1}$. For each core surface of the fourth level, select a copy (Figure 6) with the collar coordinate of its horizontal part $t=1 / 2$. Perform on each of them the operation $\mathscr{O}_{1}$ (a) by fifth-level core discs (see section 1.2.5). The horizontal parts of the used copies of the core discs have the collar coordinate $3 / 8<t<1 / 2$ in the case of '-' doubled caps and $1 / 2<t<5 / 8$ for ' + ' doubled caps. The resulting immersed discs are contracted 1-stage capped gropes. Their regular neighborhoods are kinky handles capping the meridians to the third-level 1-handles that are in the slice $t=1 / 2$. Each of these kinky handles intersects all core discs from the fifth level whose copies were used in its construction. As described in 1.4.1, we introduce finger (or Casson) moves to push each of the fifth-level core discs off the kinky handles. The resulting (regular homotopy equivalent) immersed discs are disjoint from each other and from the above constructed family of kinky handles. These kinky handles will be used in the construction of dual capped gropes. The added Casson fingers are now going to be described.

Before the finger moves, each fifth-level core disc only intersects the contracted capped grope used by the operation $\mathscr{O}_{1}$ to replace the punctured torus for the fourth-level kink on which the given fifth-level core disc is attached. So, for a fixed fifth-level core disc the relevant part of the input tower is a neighborhood of the 1-handle for this kink. This picture is described by Figure 13, with $A$ being a piece of a fourth-level 2-handle, $B$ the (fourth level) 1-handle and $C$ a piece of the fifth-level 2-handle added to the kink. Figure 24 shows the same neighborhood after the operation $\mathscr{O}_{1}$ (a). More precisely, it shows three slices: the one to the left contains the body of the grope and each of the two to the right contains one of the doubled caps, namely the shaded area to the left-hand side of Figure 8. In each of these slices the Casson fingers added to the fifth-level core disc look like pairs of parallel curves. The same picture of the fingers persists throughout a continuum of slices, parametrized by the above defined intervals. For the collar coordinates on the boundary points, the parallel curves in the left-hand part of Figure 24 bound ribbons. For $t=1 / 2$ we have ribbons of both families of fingers visible and it is easy to arrange for them to be disjoint. In the right-hand side of Figure 24, each ribbon is connected to a punctured disc bounded by the respective copy of a component of the Hopf link (see Figure 14). Those punctured discs are each in a separate slice, as described in section 1.4.1. There, the closing ribbons for both families of the fingers have the collar coordinates equal to $3 / 8$ or $5 / 8$ (the slices (1) and (8) in Figure 14).

The fifth-level discs of the input tower modified by the Casson fingers will be the core discs of a new fifth level, i.e., the $C_{5}^{1}$. The tower $T_{5}^{1}$ is defined to be the union of a regular neighborhood of $C_{1-4}^{0}$ and a regular neighborhood of $C_{5}^{1}$ in $T_{6}^{0}$. 
1.6.2. Duals to the $C_{5}^{1}$. Now we are ready to construct the promised geometric duals. We are going to construct dual capped gropes and then contract them.

The fourth-level characteristic tori $\tau_{4}$ 's that have their collar coordinates in the interval $(3 / 4,7 / 8)$ are algebraically dual to the new core discs from $C_{5}^{1}$. From arbitrary many copies of those tori $\tau_{4}$ 's we are going to build immersed geometrically dual spheres. On each copy of a $\tau_{4}$ (which is in a separate three-dimensional slice) the operation $\mathscr{O}_{3}$ (a) is performed (see 1.2.5). The punctured duals used by this operation are all in the slice with $t=3 / 4$. Next, the operation $\theta_{5}$ connects each of the $P_{3}$ 's by pipes with four copies of an $F_{4}$ that is on the same 2-handle as the given $\tau_{4}$. For those copies of core surfaces we assume $5 / 8<t<3 / 4$. The operation $\theta_{2}$ (b) is applied to the handles of these $F_{4}$ 's, but this time the fifth-level core surfaces added by the operation are "above" the fingers and $1 / 4<t<3 / 8$. Finally, on the added copies of the fifth-level core surfaces, the operation $\theta_{2}(a)$ is performed, using copies of the sixth-level core discs with the horizontal parts also having coordinates $1 / 4<t<3 / 8$.

These immersed spheres are denoted by $D_{5}$ and in fact are 1-stage contracted capped gropes, each having a $\tau_{4}$ as the body. All $D_{5}$ 's are dual to $C_{5}^{1}$ and intersect each other in the parallel copies of some $d_{6}$ 's and $P_{3}$ 's.

Figure 25 shows the relative positions of the horizontal parts of the "elementary duals" used in the construction of a $D_{5}$. We will use the same description of (a piece) of the collar neighborhood in several more figures and the "code" of the figure is described next.

Each horizontal line represents a single three-dimensional slice $\left(\cong S^{3}\right)$, and, for some of them, the collar coordinates are recorded in the figure. The dotted lines changing slices are the connecting pipes. Each level is divided into two parts by a vertical dashed line. The part to the left of the vertical dashed line contains the horizontal parts of the elementary duals that are bounded by a longitude of a 2-handle ( $d_{n}$ 's, and $F_{n}$ 's), and the part to the right contains the duals bounded by a longitude of a scooped-out 2 handle, or 1-handle in our notation (copies of $\tau_{n}$ 's and $P_{n}$ 's). Pipes that are linked with a 2-handle are to the left of the vertical dashed line and those linked with a 1-handle to the right. Whenever a dotted path representing a pipe hits a horizontal part of a dual, the pipe intersects this dual.

In Figure 25 the shaded boxes represent the position of the Casson fingers added to the fifth level that avoid the intersections with the surgered $F_{4}$ (drawn in the boxes).

Summary. We have constructed a new fifth level in the first five levels of the input tower. The core discs of the new fifth level are in the complement of $C_{1-4}^{1}$ and in the complement of certain immersed discs in the input tower. Those immersed discs that the core discs of $C_{5}^{1}$ are avoiding will be parts of a new sixth level and of its duals. For each of the core discs of the new fifth level there is a geometric dual (denoted by $D_{5}$ ) which is an immersed sphere. The resulting family of geometric duals is not disjoint because parallel copies of the $P_{3}$ 's and $d_{6}$ 's do intersect each other. Also the exact number of needed copies of each dual to a disc of $C_{5}^{1}$ is not established yet. However, after the construction of the new sixth level we will be able to estimate the number of duals we will need. 
1.7. Construction of the sixth level. We will find an embedding of a new sixlevel Casson tower $T_{6}^{1}$ inside $T_{6}^{0}$, such that the induced map from $\pi_{1}\left(T_{6}^{1}\right)$ into $\pi_{1}\left(T_{6}^{0}-T_{6}^{1}\right)$ is trivial. The standard loops on $T_{6}^{1}$, that are a basis of $\pi_{1}\left(T_{6}^{1}\right)$, will be capped by disjoint immersed discs. Those immersed discs will also have geometric duals in $T_{6}^{0}-T_{6}^{1}$. This construction will be a proof of the Big Reimbedding Theorem.

1.7.1. Loops of $C_{5}^{1}$. The new self-intersections of a fifth-level disc are the intersections of the two intersecting families of Casson fingers. Recall that those fingers were added to the core discs from $C_{5}^{0}$ in order to avoid the intersections between the core disc and its parallels. For now, we fix a fifth-level kinky handle of the input tower and we will construct a loop for each intersection of the added Casson fingers.

Recall from section 1.4.2 that all the loops for a family of intersecting Casson fingers with trivial integral intersection numbers can be constructed from the following pieces.

(1) For each intersection point on a Casson finger, there is an arc on that finger which starts at the intersection point and retraces the finger to its attaching circle.

(2) The attaching circles of every pair of intersecting Casson fingers are connected on the core disc by a ("connecting") arc.

For each intersection point, we take the appropriate pieces together to form a loop on $C_{5}^{1}$. Then, as described in Remark 1.1.1, we will isotop all the loops on $C_{s}^{1}$ to a disjoint family of (standard) loops in the boundary of the new fifth level.

We start the construction of loops with the pieces from (2). Figure 26(a) represents a piece of one of the two slices in the right hand part of Figure 24 . Compare this figure with Figure 3(a). Both intersections of the core disc and its parallel from Figure 3(a) were removed by Casson fingers. In Figure 26(a) one can see where the replacing Casson fingers were added to the core disc. In those two slices the fingers are visible as pairs of parallel curves. Each Casson finger follows an arc on the parallel (the shaded area in Figure 26(a)) until it reaches the pipe around the 1-handle immediately below. We choose a small disc on the parallel, near the beginning of the pipe. For each Casson finger added to the kinky handle there is a path in the boundary of the kinky handle which connects the attaching circle of the finger with the chosen 2-disc. Each of these paths has the following property: for each intersection point removed by a finger, a loop is formed that contains the path that starts at this point and the path followed by the finger that removed the given intersection point. The remaining end points of this paths are near the fixed disc, where they are connected by an arc. A loop constructed in this way bounds a once punctured disc in the complement of the core of $T_{5}^{1}$. The boundary of the puncture is the attaching circle of the sixth-level kinky handle added to the kink from which the Casson finger (and the given path) started.

In the (b) part of Figure 26 one can see a piece of the same neighborhood of a (positive) kink as in (a), but in a different slice. Pieces of two such paths are visible in the figure as dashed lines. Each of these paths is constructed in the following way. First the path followed by the Casson finger is projected "down" by increasing the "collar coordinate" until it reaches the slice where the original 
core disc is visible as an embedded disc, i.e., for some $t, 1<t<7 / 8$. This slice is described by Figure 3(d). In this slice, the starting point of the finger is connected with the projected path by an arc in the neighborhood of the disc (the shaded area in Figure 3(d)). Now use Convention 1.3.1 to lift this arc back to the slice from Figure 26(b) or Figure 3(a). The result is one of two dashed arcs in Figure 26(b). For a negative kink we have the mirror image of the figures.

The arcs described above for a pair of intersecting Casson fingers are joined together in the chosen disc and form "connecting arcs". These arcs are easily arranged to be disjoint so we have now the pieces from part (2) in the above decomposition.

The pieces from part (1) are ares following the intersecting fingers, as described in section 1.4.2. These arcs are visible in the left-hand side of Figure 27. For each intersection we have designated a separate slice and we assume that each pair of arcs for an intersection is in this slice. Using convention 1.3.1 again, we project the whole loop into a single slice. The projected loop is drawn in Figure 27. In order to simplify the picture, this loop was incorrectly drawn on the fingers themselves, but the loop is actually in the boundary of a tubular neighborhood of the fingers. The three different parts are separated in the same way as the ones in Figure 24 (but are now in a single slice). Also, a thrice punctured disc bounded by the loop is visible in the figure. Each of the three pieces of the loop bounds a ribbon with a puncture.

The left-hand piece of Figure 27 contains a once punctured disc with the boundary of the puncture being a meridian of the fourth-level 2-handle and is a reproduction of Figure 19 from section 1.4.2. These three pieces of the projected loop and the three once punctured discs that they bound are connected together in the obvious way. We frame these three punctured discs such that all four boundary components have the 0 -framing. To see that this is possible note that the punctured disc on the left-hand side does not intersect the Casson fingers and that the parallel longitude of this piece of the loop is parallel to the other arc in the intersection of the slice and the finger. For the parts to the right, the existence of the 0 -framing is obvious. Note also that the projected loop in the slice of Figure 27 is the unknot. As we have shown in 1.4.2, from these properties, it follows that the loops for all new kinks of a core disc in $C_{5}^{1}$ can be made disjoint. Recall that we also use the projection from 1.3.1 to define the framing on the loop as the inverse of the framing of projected loop.

For each loop we have constructed a thrice punctured disc bounded by the loop. Two of the punctures (from Figure 26(b)) can be directly capped by the sixth-level kinky handles and the third one is a meridian of a 2-handle from the fourth level. For a given intersecting pair of Casson fingers there are two intersection points, but one is "above" the Casson fingers and the other one "below". Consequently, for the given pair of fingers, one of the two punctured discs capping the two loops has the puncture (in the left-hand side of Figure 27) "above" the Casson fingers and the other "below". In both cases we are going to construct capping kinky handles. We assume, as in section 1.4.1, that each intersection between Casson fingers is in a separate slice and that the piece of the punctured disc near the puncture is in a nearby slice, away from the slices that contain the intersections. The puncture, being a meridian to the core of a fourth-level 2-handle, is piped to a copy of the punctured dual $P_{3}$. Then the operation $\theta_{5}$ adds four copies of the $F_{4}$, the core surface on the given 2-handle. 
These core surfaces have their horizontal parts in the separate slices which are "above" the one that contains the intersection point, but still "below" the ones containing the next intersection point "above". Then, the operation $\mathscr{\sigma}_{2}$ (b) adds the copies of $F_{5}$ 's with their collar coordinates in the interval $1 / 4<t<3 / 8$. The operation $\sigma_{2}$ (a) surgers the added $F_{5}$ 's by copies of the core discs that satisfy the same condition: $1 / 4<t<3 / 8$.

Figure 28 shows how this operation can add the connecting pipes without intersecting the Casson fingers from the $C_{5}^{1}$. The added copies are "above" the fingers, meaning that the pipes run through the slices containing fingers. Because pipes are linked with either both or none of the two arcs from a Casson finger, the pipes can pass throughout the slices that contain capping ribbons without introducing new intersections. Next, the application of the operation $\sigma_{2}$ (a) with the sixth-level core discs finishes the construction of a kinky handle that bounds the puncture of a loop.

In Figure 29 two examples of such capping kinky handles are shown, corresponding to the punctures for the intersections of a pair of the Casson fingers.

Note that this construction does not produce a disjoint family of kinky handles. However, the only intersections are between the copies of same $d_{6}$ 's and not between the included copies of $P_{3}$ 's. Also, for each of those capping handles, all but two self-intersections are on the copies of $d_{6}$ 's. The exceptions are two kinks on the copy of a $P_{3}$. Recall that one of them was added in order to restore the symmetry of positive and negative kinks and so can be trivially capped by a kinky handle with an arbitrary number of kinks (see section 1.1.2). The other, nontrivial, kink is capped by a punctured disc as described by Figure 10. This puncture is a meridian of the appropriate third-level 2-handle and in Figure 29 is denoted by ' $u$ ' or ' $d$ ', depending on the position of the intersection point with the respect to the fingers. These punctured discs with the puncture ' $u$ ' or 'd' will be a piece of the seventh level.

The cores of these kinky handles will be denoted throughout this section by $f^{\prime}$.

1.7.2. Disjoint duals to the sixth level of the input tower. We start with a family of copies of all third-level core surfaces, $F_{3}$ 's. The horizontal parts of the copies of the same $F_{3}$ are all in the different slices, but each of those slices contains a copy of each $F_{3}$. The "collar coordinates" of these slices are in the same open interval centered in $1 / 2$ and such that the ribbon of slices parametrized with this interval contains no intersection points of the Casson fingers added to the fifth level. We denote this interval by $J$. (Note that $J \subset\left(\frac{3}{8}, \frac{5}{8}\right)$.) On each copy of an $F_{3}$ the operation $\mathscr{Q}_{1}$ (b) is performed, using disjoint copies of the $F_{4}$ 's which were constructed in section 1.6.1 and on which the operation $\mathscr{\sigma}_{1}(\mathrm{a})$ has already been performed (see section 1.6.1). Copies of that surgered $F_{4}$ are obtained by small changes of the collar coordinate $t$ of the $F_{4}$ together with its caps. The collar coordinate of this $F_{4}$ is $1 / 2$ and the translated copies still have their $t$ 's in the interval $J$. Recall that the Casson fingers added to the fifth level were set so that those double-surgered $F_{4}$ 's are disjoint from the core of $C_{5}^{1}$.

The obtained immersed discs are denoted by $Q$. In fact, they are twice contracted 2-stage capped gropes and they are disjoint from the core. The boundary of a $Q$ is a meridian of a second-level 1-handle, so, in the sense of 
section 1.2, they are dual to the second-level 1-handles. The mutual intersections between $Q$ 's are on the parallels of the same core discs $d_{5}$ 's that are included in $Q$ 's.

To make this family of duals disjoint we use Casson fingers. The family is ordered by first taking an arbitrary ordering of the second-level 1-handles. For each slice, this orders all the $Q$ 's that have the horizontal parts of the $F_{3}$ 's included in that slice. Then it is required that a $Q$ with its $F_{3}$ above the $F_{3}$ of some other $Q$ is "after" the other one with respect to the ordering. Then, as described in section 1.4.2, the intersections between the $Q$ 's are removed by adding Casson fingers, where the bodies of the contracted gropes are $F_{3}$ 's.

For each kink on an included copy of a fifth-level core disc $d_{5}$ in a dual, we have an intersection of the copy with all other copies of the same core disc. To remove the intersections with the copies included in a doubled cap of some other dual, a Casson finger is added to the copy of $d_{5}$ (see Figure 16). This finger is linked with the double cap. It follows a vertical part to a slice which is "above" all horizontal parts of copies of core surfaces in the doubled cap. Then, inside this slice, the finger follows a guiding path, as described by Figure 5 (see section 1.2.2). At the end of the path, the finger one more time follows a vertical line until it reaches the horizontal part of the copies in the double cap. Using Figure 14 again, the finger looks like the fingers added to the original fifth level: in a neighborhood of the horizontal part of the copies its intersection with all but two slices consists of two parallel ares with ends connected. In the remaining two slices, the intersections are ribbons bounded by those two arcs.

Figure 30 shows a neighborhood of a kink on a copy of a $d_{5}$, inside the slice that contains it. There we can see some of the added fingers as the holes in the horizontal part. The ends of the above described paths are visible in Figure 30 , but this time the figure is interpreted as the neighborhood of a kink on the doubled cap that is linked with the finger.

Note that near the handles of the included $F_{4}$ 's there are already fingers added to the fifth level core, so the newly added fingers avoid intersecting them by ending up on $F_{3}$ 's rather than on the $F_{4}$ 's. The picture of the fingers near a punctured torus of an $F_{3}$ is described by Figure 13, section 1.4.1, with $A$ being a piece of a third-level 2-handle; $B$ the (third-level) 1-handle; and $C$ a piece of the fourth-level 2-handle added to the kink. It is also reproduced in (b) part of Figure 31. Parts (c) and (d) of Figure 31 show how the newly added Casson fingers can avoid the Casson fingers added to the core, and also how they can avoid loops on the $Q$ 's.

We are still going to refer to these duals as $Q$ 's.

The other family of duals we are going to construct will be denoted generically by $R$. The body of an $R$ is a copy of a third-level characteristic torus, $\tau_{3}$, with the collar coordinate for its horizontal part in the interval $\left(0, \frac{1}{4}\right)$. The operation $\theta_{3}(a)$ adds four copies of a $P_{2}$ to each $\tau_{3}$. All copies of the $P_{2}$ 's that are used are in the slice $t=\frac{1}{4}$. The resulting punctured spheres are the dual $R$ 's and are dual to the original fourth-level core. These punctured spheres are not disjoint because we assume that pipes with disjoint and unlinked ends are already added to the punctures from Figure 7(d) (in 1.2.4).

Again, to make this family disjoint, Casson fingers are introduced. Bodies on which the fingers end are double-surgered $\tau_{3}$ 's and the ordering used on the $R$ 's satisfies the same conditions as the one for the $Q$ 's: $\tau_{3}$ 's on the different 
1-handles are ordered arbitrarily and for their parallel copies it is required that those "above" come "after" in the ordering.

The picture of the beginnings of the added Casson fingers is similar to the one for the $Q$ 's: as in Figure 30, a finger follows a connecting pipe until the boundary of a $P_{2}$, i.e., it ends near the attached third-level 2-handle. The paths followed by the pipes are shown in Figure 7(c), and one of the two arcs that represent the intersection of a finger and a slice is shown in Figure 32(a). Then, each connecting pipe follows a guiding path until it ends on a $\tau_{3}$, where we have the standard picture: Figure 13 from section 1.4.1, with $A$ being a piece of a third-level 1-handle; $B$ the (third-level) 2-handle; and $C$ a piece of the second-level 1-handle. See also Figure 31(b).

Now, we complete the construction of duals to the sixth-level core discs. On each of arbitrary many copies of the fifth-level characteristic tori, $\tau_{5}$ 's, we apply the operation $\theta_{4}(b)$. Next, the same operation, $\theta_{4}(b)$, is used on each of the added $\tau_{4}$ 's, but this time the operation uses the $R$ 's, i.e., the double-surgered $\tau_{3}$ 's. The tori $\tau_{5}$ 's, $\tau_{4}$ 's and $\tau_{3}$ 's that are used are all supposed to have their horizontal parts in slices parametrized by $t \in\left(\frac{1}{8}, \frac{1}{4}\right)$. Now we have a disjoint family of punctured spheres dual to the sixth-level core discs.

The punctures are capped by those $Q$ 's that have the collar coordinates of the included $F_{3}$ 's and $F_{4}$ 's in the interval $J \cap\left(\frac{1}{2}, \frac{5}{8}\right):$ i.e., the operation $\theta_{5}$ is applied on $P_{2}$ 's in the $R$ 's by connecting them with the copies of $F_{3}$ 's which are included in the $Q$ 's. The resulting disjoint duals to the $d_{6}$ 's are denoted by $D_{6}$ and are depicted in Figure 33.

For the construction of the seventh level we will need one more class of duals. For each $d_{6}$ from $C_{6}^{0}$ there is an additional dual that we denote by $E$. It differs from a $D_{6}$ in that, on each of two copies of a $\tau_{4}$ added to a $\tau_{5}$, the operation $\mathscr{O}_{3}$ replaces the operation $\mathscr{O}_{4}$. Consequently, each $\tau_{4}$ is double surgered by four $R$ 's. Also, we require that the $\tau_{3}$ 's, $\tau_{4}$ 's and $\tau_{5}$ 's used in the construction of the $E$ 's have their horizontal parts in the ribbon of slices parametrized by $t \in\left(0, \frac{1}{8}\right)$. We further require that the $F_{3}$ 's and the $F_{4}$ 's of the $Q$ 's we use have their collar coordinates in the interval $J \cap\left(\frac{3}{8}, \frac{1}{2}\right)$. In the other words, the $R$ 's and the $Q$ 's that are used in the construction of the $E$ 's are 'after' those used in the construction of $D_{6}$ 's, where 'after' is in the sense of the above described orderings of $R$ 's and $Q$ 's.

1.7.3. Singular Norman trick. We use the singular Norman trick to get rid of the intersections and self-intersections in the sixth level of the $f^{\prime}$ 's by connecting them with the copies of $D_{6}$ 's constructed above, as we have outlined in section 1.5. Now we have a disjoint family of immersed discs trivializing all the loops of $C_{5}^{1}$. New self-intersections of these discs come from the selfintersections of $D_{6}$ 's, so their loops are in $T_{5}^{0}-C_{1-5}^{1}$.

These discs can be taken as core discs of the new sixth level. To preserve the symmetry of the construction, we "symmetrize" these immersed discs in the same way we symmetrized the input tower: all discs are made to have the number of the positive and the negative kinks equal to $x_{6}^{\prime}$ by adding new kinks when necessary. For the number of kinks $x_{6}^{\prime}$ we can take any upper bound for the set of all numbers of kinks. These symmetrized discs are going to be the $C_{6}^{1}$ and their regular neighborhoods are kinky handles that form the top level of $T_{6}^{1}$. 
Summary. The constructed $T_{6}^{1}$ satisfies the properties of The Big Reimbedding Theorem, as stated in section 1.1.1. Properties (a) and (b) are obvious from the construction of $T_{6}^{1}$. Note that (a) also holds for the original 'input tower', i.e., before the symmetrization of $T_{6}^{0}$. That the map from (c) is an isomorphism is a consequence of (a), (b) and the existence of geometric duals for the discs of $C_{5}^{1}$, namely a copy of each $D_{5}$. The immersed spheres denoted by $E$ are geometrically dual to the core discs of the input tower and are disjoint with the core of $T_{6}^{1}$.

1.8. Construction of $C_{7}^{1}$. We finish our construction of $T_{7}^{1}$ by constructing $C_{7}^{1}$ in a way similar to the way in which $C_{6}^{1}$ was constructed: we will use the extra parallels of the sixth-level core discs to cap the loops of $C_{6}^{1}$ with the immersed discs whose mutual intersections will be removed by the singular Norman trick.

1.8.1. Loops on duals. Most kinks on a kinky handle from $C_{6}^{1}$ are the selfintersections of the copies of the dual immersed spheres $D_{6}$ 's which were connected to a single $f^{\prime}$. The remaining kinks are already capped by disjoint embedded punctured discs whose punctures are the meridians of third-level 2-handles, denoted by ' $u$ ' and 'd' in Figure 29 (see section 1.7.1).

In this section we are going to cap the remaining loops which are on the $D_{6}$ 's. Recall that those duals are contracted capped gropes that are made disjoint by adding Casson fingers. Because different duals are disjoint, any loop for a sixthlevel kink will be entirely on a single dual.

Loops on the $Q$ 's. A loop for a kink on a copy of $d_{5}$ is the same as the one described by Figure 10 from section 1.3.2. Such a loop is visible in Figure 34. The loop is capped in the same way as the one in Figure 10, but this time by an embedded punctured disc that is in a small ribbon of slices immediately "below" the slice that contains the loop, and still "above" the horizontal parts of other copies of the given $d_{5}$. The two punctures in this capping disc are the result of the loop being linked with a fifth-level 1-handle and with one of the Casson fingers added to the fifth level (see Figure 30). So, one of them is a meridian of a fifth-level 1-handle and the other one is a meridian of a core disc included in $C_{5}^{1}$. However, the second meridional hole is linked only with the core disc itself and no other copy of $d_{5}$. Consequently, we can run a pipe from this hole in the "down"-direction, with the other end "below" all other constructions in the fifth level. There, we cap the end of the pipe by a dual $D_{5}$. The meridian of a fifth-level 1-handle is capped with a copy of a sixth-level core disc, or, more precisely, with a copy of the $d_{6}$ that is attached to the 1-handle.

We have a similar situation for the intersections between the different copies of a $d_{5}$. However, these different copies can belong to different doubled caps of one or two copies of a double-surgered punctured torus of an $F_{4}$. These in turn can be pieces of different doubled caps of a punctured tori on an $F_{3}$. In these cases, any loop for the intersection must pass over the body of a contracted capped grope. In all cases, the loop consists of the two arcs that both start from the intersection point and follow the horizontal part of one of the two intersecting copies of the same $d_{5}$. Then, the arcs follow parallel pipes toward the copies of an $F_{4}$, a fourth-level core surface. The parallel pieces of the arcs that are on the pipes obviously bound a ribbon in the complement of the 
other duals and the core. When projected in a single slice "above" all involved horizontal parts, the pieces of the ares that are on the horizontal parts of $d_{5}$ 's are parallel to one of the above-described loops for a kink of $d_{5}$. The only difference is that in order to close those pieces in a loop, we have to add an end to the ribbon. Again, in the chosen slice, we can embed a twice-punctured disc, whose holes are the same as before.

The other end of the ribbon is near the (one or two) copies of the $F_{4}$ to which the given $d_{5}$ 's are piped, or more precisely, near the punctured tori which were contracted by the operation $\mathscr{O}_{1}$. The picture is described in parts (c) and (d) of Figure 31, where such loops are visible as dashed arcs. In the case that both copies of the $d_{5}$ were connected to a single copy of the $F_{4}$, the loop is completed by letting the two arcs follow the fifth-level fingers. Then, as indicated by Figure 31(c), the closed path consisting of the remaining end of the ribbon and the piece of the loop visible in the figure is linked with some of the fifth-level Casson fingers. Also, in the case of an intersection between the two different doubled caps of an $F_{4}$, this loop can be linked with the visible fourthlevel 2-handle, as in Figure 19. We can cap the loop by adding a punctured disc whose punctures are meridians of the $C_{5}^{1}$, of a fifth-level 1-handle and possibly of a fourth-level 2-handle. By constructing those punctured discs in slices near the horizontal parts of $F_{4}$, we can make those discs disjoint from each other, the $C_{1-6}^{1}$ and all Casson fingers (convention 1.3.1).

In the case that the intersecting copies of a $d_{5}$ were piped to different copies of an $F_{4}$, the two arcs are near the two copies of the $F_{4}$ 's, but after projecting in a slice they are also linked with the fingers. Then, the arcs continue (the dashed arrows in Figure 31(c)) over the parallel pipes until they end on $F_{3}$, as described in Figure 31(b) or Figure 21. In Figure 20 we can see the punctured discs, with $A$ being now a piece of a third-level 2-handle, $B$ of a 1-handle on the same level and $C$ the attached fourth-level 2-handle. Note that those punctured discs also miss the Casson fingers and are again made disjoint by constructing them in separate slices. So, a capping punctured disc differs from the ones before by having a punctured disc from Figure 20 added by a ribbon to one of the previously constructed capping discs.

Consequently, a loop on a $\boldsymbol{Q}$ bounds a punctured disc with punctures being a meridian of a fifth-level 1-handle, meridians of some or, possibly, all fifthlevel Casson fingers, a meridian of a fourth-level 2-handle, and a meridian of a third-level 1- or 2-handle.

Loops on the $R$ 's. The original self-intersections on the $R$ 's were on the beginnings of the pipes added to the punctures on $P_{2}$ 's.

The pieces of the loops on the horizontal parts of the copies of a $P_{2}$ are similar to those on $d_{s}$ 's and they bound half-open discs near the horizontal parts of $P_{2}$ 's (see Figure 32(b)). Those discs are possibly punctured by the second-level 2-handle and they end on a ribbon that follows the pipes toward a $\tau_{3}$. There we again have the picture of Figure 31(b) or Figure 21 and each loop is closed on the torus. The ribbon is glued to a disc punctured by a third-level 1- or 2-handle, i.e., the picture is again Figure 20, discussed in section 1.4.3, with $A$ as a piece of a third-level 1-handle, $B$ of a third-level 2-handle and $C$ the second level 1-handle on which $B$ was attached. As before, the punctured discs from Figure 20 are disjoint and in the complement of the core and the $D_{6}$ 's. 
Loops on fingers. Loops for intersections of Casson fingers on the $Q$ 's and $R$ 's will be constructed and capped separately, but first, a common strategy of the two constructions is described.

A loop on a pair of intersecting Casson fingers consists of three arcs: two are just modified paths followed by the intersecting fingers and their starting points are connected by the third one that is an arc on the dual. (Note that all intersections are between fingers added to the same dual.) These modifications to the paths are performed in those pieces of the paths which themselves are pieces of the longitudes of $d_{5}$ 's or $P_{2}$ 's: in other words, in the slices "above" the horizontal pieces of $d_{5}$ 's and in the slices "below" the horizontal pieces of $P_{2}$ 's. To the modified parts we add twists around the finger, as for the finger guides in section 1.2.2, so that the modified arc is unlinked with the copies of the $d_{5}$ or $P_{2}$ 's in the doubled cap. However, such arcs are linked with the core disc and all its copies that are not in the doubled cap.

The third piece of a loop on the fingers is an arc connecting the starting points of the fingers and it will be constructed from pieces parallel to the loops for kinks that are not the result of the finger moves.

In general, a kink on Casson fingers is the result of addings of several generations of Casson fingers, each generation of fingers removing intersections between the previously added fingers and other duals. An example is shown in Figure 35(a) and (b). It shows a family of three intersecting capped gropes and some of the added Casson fingers. In part (a), two intersections are already removed by finger moves, i.e., Casson fingers are added to all but the first capped grope. The pair of Casson fingers in the figure has two intersections. The figure in (b) shows one of the two intersections on the Casson fingers removed by a new pair of Casson fingers.

Because most of the loops contain arcs that follow several Casson fingers, we will split the loops on the fingers into "arc pieces" and cap each of them by an immersed half-open disc. The union of the missing pieces of the boundary of discs bounded by the "arc pieces" of a single loop, will form homotopically trivial unknots with the 0-framing (in the sense of the convention from 1.3.1). Such an unknot bounds a 2-handle inside the complement of the $T_{6}^{1}$ in the 0 handle. The union of the core of this 2-handle and of the immersed half-open disc bounded by "arc pieces" of a single loop will be a capping immersed disc for the given loop.

The points where a loop is cut into the "arc pieces" are near intersections between duals that have been removed by the Casson fingers. So we have the following description of a loop on the fingers. Each of two arcs that is on the fingers starts from the self-intersection point that is near the body of an $R$ or a $Q$. Then each arc retraces backwards one of the two intersecting fingers until it reaches either the original dual $\left(d_{5}\right.$ or $\left.P_{2}\right)$ to which the given finger was added or a neighborhood of a removed intersection. In Figure 35(b), for each of the two intersections of the newly added fingers, the left-hand arc ends on a cap of the dual on the top and the right-hand arc ends on the "elbow", i.e., near the beginning of the finger introduced in part (a), that is, in a neighborhood of the removed intersection. In any case, those two arcs are the first "arc piece". Then, in the case that an arc from the first "arc piece" ends on a finger, this arc continues over the finger toward the beginning of the finger. As in Figure 35(b), 
the beginning of the retraced finger is itself added to a finger from a previous generation of fingers, and the loop again continues until a neighborhood of a removed intersection is reached. Such an arc will be also an "arc piece". After possibly several such "arc pieces", both arcs end on the caps of the dual, where they are connected together by a path on the dual which is going to be the last "arc piece". Finally, the union of the missing pieces of boundaries of the halfopen punctured disc that bounds the "arc pieces" of a single loop is capped too. This strategy of cutting a loop into pieces and capping them separately is illustrated in Figure 35(c).

Now we deal with a loop on fingers added to a $Q$. Here is a description of an "arc piece" and a half-open punctured disc bounded by it. The end points of an "arc piece" are near the intersection points between pairs of copies of the same $d_{5}$. However, the end points of "arc pieces" that belong to the same loop can be on different $d_{5}$ 's. Consequently, a single "arc piece" near an $F_{3}$ looks like the one in Figure 19. This "arc piece" is continued by two arcs onto one or two $F_{4}$ 's. These arcs can diverge to different punctured tori and they end near two kinks on some $d_{5}$ 's. Each of these diverging arcs is made to bound a punctured ribbon, roughly by taking its copy up inside the same respective slice so that this copy is unlinked with the core. One such arc on a copy of an $F_{4}$ is depicted in Figure 36. Figure 37 shows such copies that bound punctured ribbons near a torus of an $F_{4}$. In Figure 37 each number near the beginning of a diverging arc points to the cap from which the arc arrived (compare with Figure 13). The punctures of a ribbon are linked with a meridian of the fourth-level 1-handle and (or) of a 2-handle.

Near the $d_{5}$ 's, each arc follows the guiding path from Figure 5 and, because this path is unlinked with the core of this fifth-level 2-handle, the punctures in this piece of the ribbon are copies of possibly all meridians of the 1-handles attached to the 2-handle. Although such an arc ends near a clasp on a $d_{5}$, its parallel is extended so that when it continues on the next "arc piece" this parallel is still unlinked from all the handles. So, near a common end point of two "arc pieces", the extension of one of two associate parallels results in one more puncture on the ribbon for one of them, and this puncture is homotopic to a meridian of a fifth-level 1-handle. The same construction is done for all "arc pieces", even in the case that two arcs do not diverge on an $F_{4}$ and end on the same $d_{5}$.

The last "arc piece" is a path that connects the beginnings of two Casson fingers added directly to the doubled caps of the "original" $Q$. Those fingers could have been added to the same doubled cap, so this path may be completely on a $d_{5}$ or it can run over one $F_{4}$ or over two $F_{4}$ 's and an $F_{3}$. Because intersections of Casson fingers in this construction always come in \pm pairs, this connecting path can be arbitrary. However, we choose to construct this path the same way the other "arc pieces" were: each of the two beginnings of the Casson fingers is connected by a path with a single point on the appropriate doubly surgered punctured torus on an $F_{3}$. So these paths are defined the same way the "arc pieces" were.

Finally, adding all the ribbons and half-open discs bounded by "arc pieces" of a single loop gives a punctured half-open disc bounded by the loop. The missing part of the boundary is the union of the remaining pieces of the boundaries of 
the punctured ribbons and half-open discs. Using our convention from section 1.3.1, we can project all these pieces into a single slice, which we choose to be immediately "below" all slices containing those pieces. There, the resulting loop is unknotted and unlinked from the rest of the construction. For each loop we select a different slice until all loops are capped. So all loops for the kinks on the fingers added to $Q$ 's are capped by disjoint punctured discs.

The loops on the Casson fingers added to the $R$ 's are capped in a similar way. We use the same process of cutting a loop into "arc pieces" which are capped separately. The first "arc piece" again consists of two arcs, both starting from a common point near the intersection of a pair of fingers near a thirdlevel characteristic torus. There the picture is the one in Figure 19, with $B$ representing a third-level 2-handle and $C$ a second-level 1-handle. Near the copies of a $P_{2}$ (parallel to the 1-handle $C$ in Figure 19), the arcs are following the same 'guiding paths' as the connecting pipes and added Casson fingers did (Figure 7(c)). Recall that those pipes are in the slices "below" the one that contains the horizontal parts of the involved $P_{2}$ 's and that each pipe ends in a separate slice. The ends of these two arcs are near components of the clasps of $P_{2}$ 's. These two ends are connected by a path which is unlinked from $C_{1-6}^{1-6}$ and the $P_{2}$ 's. Figure 32(a) describes those fingers and arcs. The other "arc pieces" consist of an arc that looks the same as the first "arc piece" (see Figure 35) and a similar connecting path is added to each. Using again the convention from section 1.3.1, each of the "arc pieces" and added paths is in a separate slice "below" the one containing the horizontal parts of the $P_{2}$ 's. Each "arc piece", together with the added path, bounds a punctured disc; one puncture is a meridian of the third-level 1-handle (the component $A$ in Figure 19) and possibly, the other one or two are meridians of the second-level 2handle to which the 1-handle containing the given $P_{2}$ 's was attached. These latter punctures occur when the two arcs have different linking numbers with the second-level 2-handle.

Again, the last "arc piece" is a path that connects the beginnings of a pair of Casson fingers added directly to one or both of the doubled caps of the "original" $R$. This path consists of two arcs that start from the beginnings of the pair of fingers and follow appropriate horizontal parts of one or two copies of the $P_{2}$. In Figure 32(b) they are represented by dashed arrows that end on the boundaries of the $P_{2}$ 's. Then, the arcs follow the parallel pipes that connect these boundaries with a copy of a $\tau_{3}$. There, they are connected in a common point and we have one of the situations described by Figure 31(b).

The family of loops for the kinks of a $D_{6}$ that we obtain is a 'standard family'. To show this, we use Lemma 1.3.2. All loops are grouped in ordered \pm pairs by first taking an ordering of the \pm pairs of loops on the fingers and then an ordering of the remaining \pm pairs. A \pm pair of loops on the fingers corresponds to a \pm pair of intersections between a pair of Casson fingers. The remaining \pm pairs are formed by using the symmetry between positive and negative kinks on a $P_{2}$ or a $d_{5}$. The ordering of the pairs of loops on the fingers is arbitrary (see section 1.4.2) and the remaining pairs can be ordered as described in section 1.4.3 and by Figure 22.

1.8.2. Fake seventh level. Now we have a 'standard family of loops' that bound imbedded disjoint punctured discs inside $T_{6}^{0}-C_{1-6}^{1}$. The punctures are 
meridians to the 1- and 2-handles of $T_{2-5}^{1}$ and we are going to cap them by kinky handles.

There are many ambiently isotopic copies of each of the punctures we are going to cap by the same construction, using small changes in the "collar coordinates". Intersections between those immersed discs that cap such copies and that are on included punctured duals $P_{n}$ 's are going to be traded by the singular Norman trick for new self-intersections: each set of two copies of the same $P_{n}$ has four intersection points, a pair for each kink of the $P_{n}$. To remove such an intersection, a finger is added to one of the copies of the $P_{n}$. This finger starts from a small disc that contains the intersection point in the first copy and then the finger "moves" this disc over a path on the second copy towards the boundary of the immersed disc that contains the second copy of the $P_{n}$. As described in [FQ, section 2.5], after the boundary of the immersed disc (that is on the new sixth level), the finger splits to a pair of fingers that end on the boundary of the sixth level. There, fingers split again, and, on the resulting four fingers on the $C_{5}^{1}$, the singular Norman trick is performed by connecting each "tip" of a finger with a copy of a $D_{5}$. So, this process removes an intersection point between two copies of the same $P_{n}$ by adding four duals $D_{5}$ to one of the copies. To the copy of the $P_{n}$ with the horizontal part "above" the horizontal part of the other copy of the $P_{n}$, four copies of a $D_{5}$ are connected by the described procedure to each of the two intersections that are in a neighborhood of the positive kink on the $P_{n}$. Notice that the sign of these two intersections depends on the induced orientations on the copies of the $P_{n}$, but the signs are irrelevant for this construction and are used only to group the intersections in two pairs. To the copy of the $P_{n}$ with its horizontal part "below", four copies of a $D_{5}$ are connected to each of the two remaining intersection points, i.e., to those that are induced by the negative kink of the "original" $P_{n}$. Therefore, to each copy of a $P_{n}$ that is included in a capping kinky handle for a puncture, we add eight times as many copies of a $D_{5}$ as there are other isotopic parallels of the $P_{n}$ in the capping kinky handles. If there are $k$ many copies of a $P_{n}$ in the capping kinky handles, the total number of $D_{5}$ 's we need is $8 k(k-1)$.

Figure 38 describes positions in the 0-handle of kinky handles capping the punctures of the capping discs for the loops on the $Q$ 's. The punctures are meridians of the fifth-level 1-handles, meridians of some or, possibly, all fifthlevel Casson fingers, meridians of the 1-handles and 2-handles of the third and fourth levels. Also the figure describes the way the puncture denoted by ' $d$ ' in section 1.7.1 is capped (see Figure 29).

The meridians of the fifth-level Casson fingers are all piped to copies of $D_{5}$ 's, because they are not linked with anything else other than the new fifth-level core discs. A meridian of a fifth-level 1-handle is immediately capped by a copy of the $d_{6}$ that is attached to the 1-handle.

The punctures that are meridians of the fourth- and third-level 2-handles are capped in the following way: in a slice that is "above" the one containing the puncture, a meridian is connected to a punctured dual on the one level below (the third or the second, respectively). Then the operation $Q_{2}$ (b) adds further core surfaces, either $F_{4}$ 's or $F_{3}$ 's and $F_{4}$ 's, respectively. The added punctured duals are in nearby slices. Then, in both cases, to $F_{4}$ 's the $\sigma_{2}(b)$ adds $F_{5}$ 's, but this time in slices that are "above" the fingers, or more precisely, in the 
ribbon of slices $t \in\left(\frac{1}{4}, \frac{3}{8}\right)$. Those $F_{5}$ 's are surgered by $\theta_{2}$ (a) with copies of $d_{6}$ 's that have their horizontal parts in the same ribbon of slices. However, the used punctured duals intersect the pipes connecting the $P_{2}$ 's from the $R$ 's with $F_{3}$ 's in $Q$ 's, or those pipes connecting $F_{3}$ 's with $F_{4}$ 's (Figure 38). In both cases these pipes are pieces of the $D_{6}$ 's and not of the $E$ 's. Also, kinky handles that cap copies of the same meridian have intersections in the included copies of the same $P_{2}$ 's. The singular Norman trick is used to remove all these intersections by piping them to $D_{5}$ 's with pipes that run over $D_{6}$ 's, $f^{\prime}$ 's and $C_{5}^{1}$. Intersections between $P_{2}$ 's are removed as described above, and all others by adding duals $D_{5}$ 's to the capping kinky handles.

The punctures denoted by 'd' in section 1.7.1 and Figure 29 are meridians to the third-level 2-handles and are capped the same way the others were.

Finally, a puncture which is a meridian of a third- or fourth-level 1-handle is capped by a copy of an $F_{3}$ or $F_{4}$ which is in a nearby slice. In the case of a third-level meridian, the application of operation $\theta_{2}(b)$ adds $F_{4}$ 's also in the slices near the one containing the puncture. Then, the operation $\mathcal{O}_{2}(\mathrm{~b})$ adds two copies of an $F_{5}$ to each punctured torus on an included copy of an $F_{4}$. As in the construction above, the horizontal parts of the $F_{5}$ 's are in the ribbon of slices $t \in\left(\frac{1}{4}, \frac{3}{8}\right)$. The operation $Q_{2}$ (a) surgers each $F_{5}$ with copies of $d_{6}$ 's which are in the same ribbon of slices $t \in\left(\frac{1}{4}, \frac{3}{8}\right)$.

The punctures on the punctured discs bounded by the loops on the $R$ 's are meridians of the second-level 2-handles (see Figure 32) and of the third-level 1and 2-handles. Also the puncture denoted by ' $u$ ' in Figure 29 is capped.

The capping punctured discs differ from those for the $Q$ 's in that near the copies of the $P_{2}$ 's they are now "above" the slice with the horizontal parts of the $P_{2}$ 's (and the added Casson fingers are "below" the $P_{2}$ 's this time).

Figure 39 shows how the punctures are capped. The punctures near the $P_{2}$ 's are meridians of the second-level 2-handles and are each capped by a copy of a $P_{1}$. All copies of $P_{1}$ used have $t=\frac{1}{4}$. The operation $\theta_{5}$ adds four $F_{2}$ 's. Three applications of $\mathscr{O}_{2}$ (b) and one of $\mathscr{O}_{2}$ (a) finish the construction of capping kinky handles and all core surfaces involved are in the ribbon of slices $t \in\left(\frac{1}{4}, \frac{3}{8}\right)$. To remove the intersections between the copies of the same $P_{1}$ in different kinky handles, the singular Norman trick is used again with copies of the $D_{5}$ 's.

The punctures that are third-level meridians to 2-handles are each piped to a copy of a $\tau_{2}$. The horizontal parts of the copies of these $\tau_{2}$ 's have the collar coordinates in the interval $\left(\frac{1}{8}, \frac{1}{4}\right)$. Then, the $\tau_{2}$ 's are 'double-surgered' by each of four copies of $P_{1}$ 's, and the rest of the construction is the same as in the paragraph above. However, all intersections between different copies of the kinky handles capping the third-level meridians are removed by Casson fingers that end near $\tau_{2}$ 's, the same way as it was done for the $R$ 's. Other intersections are removed by adding $D_{5}$ 's.

For the meridians of the third-level 1-handles, the connecting pipes run "downward", intersecting each $\tau_{3}$ from an $R$ in a point that is in its horizontal part. Then, "below" all $R$ 's, where $\frac{1}{4}<t<\frac{3}{8}$, the pipes are closed by $F_{4}$ 's, which are surgered by $F_{5}$ 's (the operation $\theta_{2}(b)$ ) and finally, the $F_{5}$ 's are surgered by $d_{6}$ 's. Those capping kinky handles are not disjoint and they intersect the sixth level (in $\tau_{3}$ 's). Using the singular Norman trick again with 
copies of $D_{5}$ 's those intersections are traded for new self-intersections on $D_{5}$ 's.

The puncture ' $u$ ' is capped in the same way as the other third-level meridians of the 2-handles, but this time the meridian is piped "through" the horizontal parts of all $F_{3}$ 's that are included in the previously constructed capping kinky handles. Each of these intersections is removed by the singular Norman trick, i.e., copies of a $D_{5}$ are added to the $F_{3}$ 's.

After all punctures are capped, we have new intersections between the copies of $D_{5}$ 's used by the singular Norman trick. They are all traded for new selfintersection by introducing Casson fingers on the doubled caps of $D_{5}$ 's. The required ordering of the involved copies of $D_{5}$ 's is arbitrary.

Although the constructed capping kinky handles do intersect each other, all intersections between them are now only intersections between copies of the same $d_{6}$ 's which are included in the different kinky handles.

Note that the selection of the "topmost" $Q$ 's and $R$ 's for the construction of the $E$ 's ensures that the 'fake seventh level' intersects $E$ 's only in $\tau_{5}$ 's, i.e., each copy of a $d_{6}$ in the fake seventh level intersects each $\tau_{5}$ in a point. In another words, this 'fake seventh level' has 'duals' in the sense of Remark 1.5.

1.8.3. Singular Norman trick again. Now we are in a situation similar to the one in section 1.7.3. We have a family of immersed discs with geometric duals, but neither the discs nor the duals are disjoint from each other. Those duals are the $E$ 's which are 1-stage capped gropes and the body of each of them is a $\tau_{5}$ surgered by two copies of a $\tau_{4}$ (see section 1.7.2).

Again, after we make sure that we have enough parallel copies of each $E$, we can contract them one by one to obtain a disjoint family of the geometrically dual spheres. After applying the singular Norman trick to trade the intersections and self-intersections of the "capping discs" that are on the copies of $d_{6}$ 's, we have disjoint immersed discs capping all the loops of the $C_{6}^{1}$, and this is the $C_{7}^{1}$.

Now, a small regular neighborhood of $C_{(1-7)}^{1}$ will be a seven-level Casson tower $T_{7}^{1}$ having properties (i) and (ii) from the reimbedding theorem in section 1.1.1.

\section{Estimates}

In this section we will estimate the number of kinks in the core of the embedded tower $T_{7}^{1}$.

2.0. Notations. Recall that we have denoted caps of a capped grope by $c_{i}^{ \pm}$, meaning that the top stage of a grope had " + " and " - " caps [FQ, section 2.6] and the index $i$ comes from an indexing of the gropes. An expression like " $\pitchfork c_{i}^{ \pm}=(p, n)$ " will mean that the caps $c_{i}^{ \pm}$have $p$ positive and $n$ negative self-intersections, and by " $c_{i}^{ \pm} \pitchfork c_{j}^{ \pm}=(a, b)^{\text {n }}$ we will indicate that there are $a+b$ intersections between $c_{i}^{ \pm}$and $c_{j}^{ \pm}$of which $a$ are positive and $b$ negative. So, by the 'intersection number' and the 'self-intersection number' we will always mean an ordered pair of nonnegative integers.

Remark 2.0. To simplify our estimates we will assume that the numbers of kinks on the branches of the input tower, $x_{2}, \ldots, x_{6}$ are all the same and we denote this number by $x$. In other words, we assume that the input tower is symmetrized and that all the core discs, except for the one on the first level, 
have $x$ positive and $x$ negative kinks. This can be obtained by taking for $x$ an upper bound of the numbers of positive and negative kinks. Then extra kinks are added where needed (see section 1.1.2).

2.1. Kinks on contracted capped gropes. To estimate the numbers of kinks on $T_{7}^{1}$, we will need estimates for the numbers of intersections and self-intersections on the capped gropes used in the construction of $C_{(6-7)}^{1}$. In our case, we have a simpler situation than in general, because all our intersecting caps are, essentially, parallel copies of each other. So, the intersection and the selfintersection numbers are the same for all caps and we get simpler formulas for the intersection numbers of the resulting immersed spheres.

To calculate the intersection and self-intersection numbers after a contraction of a capped grope, we need to know the numbers of intersections there were between the caps of the grope, and between the caps and the other surfaces that we want to push off those caps. If $c G^{1}$ is a properly immersed 1-stage capped grope such that its body is a genus 1 surface, possibly with boundary, and such that for some positive integer $k$ the intersection numbers of its caps are: $\pitchfork c^{ \pm}=(k, k)$ and $c^{+} \pitchfork c^{-}=(2 k, 2 k)$, then the resulting immersed sphere, $G^{0}$, will have self-intersection number $\pitchfork G^{0}=4 \cdot\left(\pitchfork c^{+}+c^{+} \pitchfork c^{-}+\pitchfork c^{-}\right)$, so

$$
\pitchfork G^{0}=(16 k, 16 k) \text {. }
$$

Suppose that there is a family, $c G_{1}^{1}, \ldots, c G_{n}^{1}$, of $n$, properly immersed, 1-stage capped gropes with genus 1 bodies and with the intersection numbers of their caps being $\pitchfork c_{i}^{ \pm}=(k, k)$ and $c_{i}^{ \pm} \pitchfork c_{j}^{ \pm}=(2 k, 2 k)$ for $1 \leq i, j \leq n$ and some positive integer $k$. If these gropes are all contracted, then the resulting immersed spheres $G_{1}^{0}, \ldots, G_{n}^{0}$ will have their self-intersection numbers given by formula (1). It is easy to see that for $1 \leq i, j \leq n$ we have

$$
G_{i}^{0} \pitchfork G_{j}^{0}=(32 k, 32 k) \text {. }
$$

To make the family of spheres $G_{i}^{0}, 1 \leq i \leq n$, disjoint, one can push the first contracted grope off the caps of all other gropes. This process introduces new intersections and self-intersections between the doubled caps of $G_{i}^{0}(2 \leq i \leq n)$, which are coming from two families of Casson fingers piercing each other. Next, the new contracted gropes $G_{i}^{0}(3 \leq i \leq n)$ are pushed off $G_{2}^{0}$. This process is repeated $(n-1)$ times. The result is a disjoint family of contracted capped gropes. The new gropes are regularly homotopic to the old ones and are still denoted by $G_{i}^{0}$.

Before the contractions, the total number of intersections was $\left|c_{i}^{ \pm} \pitchfork c_{1}^{ \pm}\right|=$ $4 k$ (for $i, j>1$ ). After the Casson moves there are $4 k \pm$ pairs of Casson fingers starting from each copy of a cap included in a doubled $c_{i}^{ \pm}$. Recall that each Casson finger avoids (or is linked with) one of the two doubled caps of $G_{1}^{0}$ rather than just one copy of a $c_{1}^{ \pm}$. We have arranged for Casson fingers that start from the same cap and are near one of the $c_{1}^{ \pm}$to be disjoint. Also, any two fingers that are linked with different doubled caps intersect each other in $2(4 k)^{2}$ points, half of them with the + sign.

The new intersection and self-intersection numbers on copies of $c_{i}^{ \pm}$in the doubled caps of $G_{i}^{0}(i, j>1)$ are
(3)
m $c_{i}^{ \pm}=\left(k+16 k^{2}, k+16 k^{2}\right)$,

$$
c_{i}^{ \pm} \pitchfork c_{j}^{ \pm}=\left(2 k+32 k^{2}, 2 k+32 k^{2}\right)
$$


Notice that the intersection numbers are still twice the self-intersection numbers. Hence, for a contracted $c G_{i}^{1}$, in the formula for the self-intersection number, the 'input' is $k+16 k^{2}$ instead of $k$. Consequently, after pushing the $G_{1}^{0}$ off all other $G_{i}^{0}$ 's, for $i, j \geq 2$, we have

$$
\pitchfork G_{i}^{0}=\left(16 k+(16 k)^{2}, 16 k+(16 k)^{2}\right), \quad G_{i}^{0} \pitchfork G_{j}^{0}=2 \cdot \pitchfork G_{i}^{0} .
$$

If we repeat this process $n$ times, we will run into the difficulty of calculating the resulting polynomial, namely, the one obtained by applying the function $k \mapsto k+16 k^{2} n$ times. We can approximate the $n$-fold composition of the function $k \mapsto k+16 k^{2}$ with itself by the polynomial

$$
g_{n}(k)=k(17 k)^{\left(2^{n}-1\right)} \text {. }
$$

Because we shall always simplify our towers by making all intersection numbers equal throughout the whole level, we will always take the biggest number (or any upper bound) as the number of the intersections of all spheres obtained by the iterated contractions of a family of the capped gropes. With the data as above, after the $n$ contractions we have:

$$
\pitchfork G_{i}^{0}=\left(16 k(17 k)^{\left(2^{n}-1\right)}, 16 k(17 k)^{\left(2^{n}-1\right)}\right) \text { for all } 1 \leq i \leq n .
$$

2.2. Number of kinks on $C_{5}^{1}$. In section 1.6.1 we have constructed $C_{5}^{1}$ by changing the discs $d_{5}$ 's of $C_{5}^{0}$ by Casson moves. Those Casson fingers avoided the $Q$ 's or, more precisely, the parallels to the $d_{5}$ 's included in the $Q$ 's. We have assumed $\pitchfork d_{5}=(x, x)$, so:

$$
c_{i}^{ \pm} \pitchfork d_{5}=(2 x, 2 x) ; \pitchfork c_{i}^{ \pm}=(x, x) \text {. }
$$

This pushing of the fifth-level core discs off the $Q$ 's, results in new fifthlevel core discs that have new self-intersections coming from $\left|c_{i}^{ \pm} \pitchfork d_{5}\right|$-many pairs of Casson fingers. The new intersections thus arrive in pairs and from formula (3) it follows that the total number of self-intersections for discs in $C_{1}^{5}$ is $\left(x+16 x^{2}, x+16 x^{2}\right)$.

All Casson moves of a $d_{5}$ are localized around the $Q$ 's, so we have not introduced any intersections between different new $d_{5}$ 's and so we have a new fifth-level tower. Obviously, we have a new $x$ for the fifth level, which we will denote by $x_{5}^{\prime}$, and which is equal to $x+16 x^{2}$. To make calculations easier, we will approximate $x_{5}^{\prime}$ by $(10 x)^{2}$ by adding extra kinks, if necessary. So we set

$$
x_{5}^{\prime}=(10 x)^{2} .
$$

2.3. Number of kinks on $C_{6}^{1}$. First we are going to estimate the number of parallels of each $d_{6}$ that are needed to cap a loop of $C_{5}^{1}$. Then we estimate the number of self-intersections of each $D_{6}$ from section 1.7.2, and finally, we estimate the number of kinks of $C_{6}^{1}$, denoted $x_{6}^{\prime}$.

Because the dual $D_{6}$ 's are situated in levels 2 to 6 and because those that contain copies of different $P_{2}$ 's are disjoint, all our estimates are going to be for discs, duals and loops over a single second-level 1-handle.

2.3.1. 'New' loops of $C_{5}^{1}$. The kinks on the added Casson fingers were capped in section 1.7 .1 by an intersecting family of kinky handles. We started with 
thrice punctured discs. Two of the punctures on each disc are meridians of the sixth-level 1-handles and are directly capped by copies of the appropriate $d_{6}$ 's (see Figure 24). The third puncture on each disc is a meridian to a fourth-level 2-handle and is capped by kinky handles which have intersections in copies of $d_{6}$ 's (see Figure 29). Such a kinky handle was constructed by adding four copies of an $F_{4}$ to a $P_{3}$, then each handle on an $F_{4}$ was surgered by two $F_{5}$ 's, and, finally, each of the handles on the fifth level was surgered by two copies of a $d_{6}$. So, each of these kinky handles contains the total of $64 x^{2}$ copies of $d_{6}$ 's. In total, we cap each loop on a pair of Casson fingers by $\left(2+64 x^{2}\right)$ copies of $d_{6}$ 's.

Each 'old' loop can be capped by a single copy of a $d_{6}$, but to preserve the symmetry of the numbers of kinks we will add kinks to their capping kinky handles.

2.3.2. Number of $D_{6}$ 's. The number of needed $D_{6}$ 's is equal to the product of the number of copies of $d_{6}$ 's used in the sixth level and the number of loops on the fifth level.

After the approximation of $x_{2}, \ldots, x_{5}$ by $x$, there are $4 x^{2}$ of fifth-level discs over each second-level 1-handle and each has $\left(2 x+32 x^{2}\right)$ loops. So, in capping the loops of $C_{5}^{1}$ that are over a second-level 1-handle, we have used $4 x^{2}\left(2 x+32 x^{2}\right)\left(2+64 x^{2}\right)$ copies of $d_{6}$ 's. For each copy of a $d_{6}$ we need $2 x$ duals, so the total number of $D_{6}$ 's over a second-level 1-handle we need is

$$
8 x^{3}\left(2 x+32 x^{2}\right)\left(2+64 x^{2}\right) \text {. }
$$

It does not hurt to use more $D_{6}$ 's than needed, so we agree to use $n$ of them, where

$$
n=1.1 \cdot(4 x)^{7}
$$

Note

$$
8 x^{3}\left(2 x+32 x^{2}\right)\left(2+64 x^{2}\right)=4^{7} \cdot\left((1 / 512) x^{4}+(1 / 32) x^{5}+(1 / 16) x^{6}+x^{7}\right),
$$

so $8 x^{3}\left(2 x+32 x^{2}\right)\left(2+64 x^{2}\right)<n$ for $x \geq 1$.

2.3.3. Self-intersection number of $a D_{6}$. Each $D_{6}$ contains four $R$ 's and 64 $Q$ 's, so there are $4 n$ copies of $R$ 's and $64 n$ copies of $Q$ 's included in those $D_{6}$ 's. Each of these two families is made disjoint by introducing finger moves.

Because $\pitchfork P_{2}=(1,1)$, from $(5)$ we have the following estimate:

$$
\text { ก } R=\left(17^{2^{(4 n)}}, 17^{2^{(4 n)}}\right)
$$

An application of the operation $\mathscr{O}_{1}(\mathrm{~b})$ on a copy of an $F_{4}$ in a $Q$ produces an immersed disc, which we temporarily denote by $c^{ \pm}$. According to formula (1), we have: $\pitchfork c^{ \pm}=2 x(16 x, 16 x)=\left(32 x^{2}, 32 x^{2}\right)$.

Using this number as the input in formula (5), the self-intersection number of a symmetrized $Q$ with all its Casson fingers added is:

$$
\begin{aligned}
& \text { † } Q=2 x\left(16\left(17 \cdot 32 x^{2}\right)^{\left(2^{64 n}-1\right)}, 16\left(17 \cdot 32 x^{2}\right)^{\left(2^{64 n}-1\right)}\right) \text {. } \\
& \text { i } Q=\left(32 x \cdot\left(17 \cdot 32 x^{2}\right)^{\left(2^{64 n}-1\right)},-\right) \text {. }
\end{aligned}
$$


Finally, the self-intersection number for a $D_{6}$ is

$$
\pitchfork D_{6}=4(\pitchfork R)+64(\pitchfork Q) \text {. }
$$

Let us denote the components of the pair $\pitchfork D_{6}$ by $z$ (i.e., $\pitchfork D_{6}=(\bar{z}, \bar{z})$ ). Since we can introduce more kinks at will, it is enough to estimate $z$, and we use $z$ to denote the actual number of kinks we will take. In our case $n$ will always be bigger than 2 , so

$$
\begin{aligned}
\bar{z} & =4(17)^{2^{4 n}}+64 \cdot 32 x\left(17 \cdot 32 x^{2}\right)^{\left(2^{64 n}-1\right)} \\
& <(64 \cdot 32 x+1)(17 \cdot 32 x)^{\left(2^{64 n+1}-2\right)}<(17 \cdot 32 x)^{2^{64 n+1}} \\
& <(10 x)^{2^{64 n+3}} .
\end{aligned}
$$

Now $2^{64 n+3}<2^{66 n}=\left(2^{3.3}\right)^{66 n / 3.3}<10^{20 n}$, so we can take

$$
z=(10 x)^{10^{\left(22(4 x)^{7}\right)}} \text {. }
$$

2.3.4. The $x_{6}^{\prime}$. To the number of loops on the $D_{6}$ 's, we also have to add the number of kinks on $P_{3}$ 's that are included in the immersed discs capping the intersections of the Casson fingers added to the fifth level (see Figure 29). Because these capping discs contain disjoint copies of $P_{3}$ 's and because each of them contains only one copy of a $P_{3}$, they will contribute 1 to $x_{6}^{\prime}$.

Each loop of $C_{5}^{1}$ has $\left(64 x^{2}+2\right) \cdot 2 x$ copies of various $D_{6}$ 's included, and hence contributes

$$
\left(64 x^{2}+2\right) \cdot 2 x \cdot z+1
$$

By introducing more kinks, we can take

$$
x_{6}^{\prime}=(10 x)^{10\left(x(10 x)^{6}\right)}
$$

since $\left(64 x^{2}+2\right) \cdot 2 x \cdot z+1<z^{2}<x_{6}^{\prime}$.

2.4. Number of kinks on $C_{7}^{1}$. To find $x_{7}^{\prime}$, we need to estimate an upper bound for the number of $D_{s}$ 's and the number of $E$ 's connected by the singular Norman trick to an immersed disc that caps a loop in $C_{6}^{1}$. Also, we need estimates for the self-intersection numbers of the $D_{5}$ 's and the $E$ 's.

A $D_{5}$ is a contracted capped grope whose body is a copy of a characteristic torus $\tau_{4}$. Each of the caps contains $64 x^{2}$ copies of $d_{6}$ 's, and a copy of a $P_{3}$. The intersections between the $D_{5}$ 's that are on the copies of the $P_{3}$ 's are removed by Casson fingers.

The other duals used in the construction of the seventh level are $E$ 's. Each of them is a 1-stage (uncontracted) capped grope with the body being a $\tau_{5}$ surgered by two copies of a $\tau_{4}$. A cap of an $E$ is an $R$ connected with 16 $Q$ 's. Note that the number of kinks on the $R$ 's and $Q$ 's that are in $E$ 's are the result of the finger moves that were used to remove their intersections with the $R$ 's and $Q$ 's that are included in $D_{6}$ 's.

Throughout this section the number of the $D_{6}$ 's that are over a second-level 1-handle and are used in the construction of the sixth level is still denoted by $n$. However, our estimates in this section will be for the numbers of kinks and surfaces over each first-level 1-handle (or in a branch of the input tower), so above each first-level 1-handle there are $2 \times n$ of $D_{6}$ 's. 
2.4.1. Number of $D_{5}$ 's. We are interested only in an upper bound for the number of $D_{5}$ 's included in a seventh-level disc. It is easy to see that the biggest numbers of $D_{5}$ 's are for the loops on Casson fingers that contain the maximal number of the "arc pieces". Comparing Figure 39 with Figure 38, one can see that the maximal number of $D_{5}$ 's will be for the loops on fingers added to the $R$ 's. Namely, the discs capping the loops on the $Q$ 's have the number of the added $D_{5}$ 's proportional to the number of $Q$ 's, and so to the $n$. On the other hand, the number of $D_{5}$ 's added to a disc over an $R$ is proportional to the total number of loops on $R$ 's, so it is a number which is greater than the $z$ from (10).

On each of the $8 \times n$ included $R$ 's that are in a given branch of the input tower, a loop is capped by a punctured disc whose punctures are possibly one or two meridians to a second-level 2-handle and a meridian to a third-level 1or 2-handle (see Figure 32). The way those punctures are capped is described by Figure 39.

A second-level meridian to a 2 -handle (near an $R$ ) is piped to a copy of a $P_{1}$, and a third-level meridian to a 2-handle is piped to a copy of a $\tau_{2}$ that is connected with four copies of a $P_{1}$ by the operation $\theta_{3}$ (a).

Because the copies of $P_{1}$ 's used in the both cases are intersecting each other, we have to trade the intersections between the immersed discs that cap meridians on the second and third levels for these new kinks. The intersections are traded by the singular Norman trick involving copies of the $D_{5}$ 's.

The remaining punctures are meridians to third-level 1-handles and each is capped by a copy of an $F_{4}$ to which the operations $\theta_{2}$ (b) and $\theta_{2}$ (a) attach $16 x^{2}$ copies of $d_{6}$ 's. These capping kinky handles have intersections only in copies of $d_{6}$ 's, but they intersect the $R$ 's in their bodies, namely in copies of $\tau_{3}$ (see Figure 39). Assuming that each capping kinky handle intersects each of the $R$ 's in a point, we estimate that to each of those capping handles we have to add $\frac{4 n}{2 x}$ copies of $D_{5}$ 's (this is the number of $R$ 's whose bodies are copies of a single $\tau_{3}$ ).

The number of $P_{1}$ 's in the core discs of $C_{7}^{1}$ is estimated to be the product of the number of loops with the maximal number of "arc pieces" and with the maximal number of the $P_{1}$ 's added to each "arc piece".

The number of loops on each $R$ times the number of the $R$ 's over a first level 1-handle is

$$
2 \cdot 17^{2^{(4 n)}} \cdot 8 x n
$$

and the number of the $P_{1}$ 's on each of the (at most) $8 n$ "arc pieces" is at most 6. So, the number of copies of each $P_{1}$ is estimated by:

$$
2 \cdot 17^{2^{(4 n)}} \cdot 8 x n \cdot 6 \cdot 8 n=3 \cdot 2^{36} x^{15} 17^{\left.2^{\left(2^{16}\right.} x^{7}\right)}
$$

An estimated number of needed $D_{5}$ 's is twice the square of this number (see section 1.8.2). Also, each "arc piece" may contain a kinky handle capping a third-level meridian to a 1-handle and such a kinky handle can intersect all bodies of the $R$ 's. That gives us $4 n \cdot 2$ additional copies of $D_{5}$ 's per "arc piece", so, in total, we have $(8 n)^{2}$ additional $D_{5}$ 's.

The pipes connecting circles denoted by ' $u$ ' in Figure 29 and Figure 39 are intersecting all copies of $F_{3}$ 's whose collar coordinates are in the interval $\left(\frac{1}{4}, \frac{3}{8}\right)$. 
These intersections are removed by a singular Norman trick that connects a copy of an $F_{3}$ near each intersection point by a pipe that runs over the given pipe that is added to a ' $u$ ', and down to the sixth and fifth levels where it ends in four copies of a $D_{5}$. The total number of meridians ' $u$ ' is equal to twice the number of the intersections of the fifth-level Casson fingers that are in the ribbon of the slices $t \in\left(\frac{3}{8}, \frac{1}{2}\right)$. This is also the total number of the intersections between the Casson fingers added to the fifth level core discs that are above a given third-level 2-handle, and so it is equal to $2 x^{2} \cdot 32 x^{2}=2^{7} x^{4}$. Because there are at most $3 \cdot 2^{5} x$ of $F_{3}$ 's in an "arc piece", we have to add to a loop $4 \cdot 8 n \cdot 3 \cdot 2^{5} x=\cdot 2^{24} x^{8}$ copies of $D_{5}$ 's.

Finally, we approximate this number by

$$
x^{15} 10^{10^{3000 x}}
$$

2.4.2. Number of $E$ 's. We estimate the number of $E$ 's in the discs capping the loops on $R$ 's and $Q$ 's separately.

An "arc piece" on an $R$ can have punctures that are one or two meridians to the second-level 2-handle and a meridian to a third-level 1- or 2-handle. Each immersed disc capping the second level meridians to the 2-handles contains $4(4 x)^{4}$ copies of $d_{6}$ 's. A third-level meridian to a 2-handle is piped to a copy of a $\tau_{2}$ that is connected with four copies of a $P_{1}$ using $Q_{3}$ (a). Consequently, $16(4 x)^{4}$ copies of $d_{6}$ 's are used for each meridian and the capping kinky handles are contracted capped gropes. A meridian to a third-level 1handle is capped by a copy of an $F_{4}$ to which the operations $\theta_{2}$ (b) and $\theta_{2}$ (a) attach $16 x^{2}$ copies of $d_{6}$ 's. So, to each "arc piece" there are less than $2^{14} x^{5}$ copies of $E$ 's attached. We approximate the number of $E$ 's in a capping kinky handle for a loop to be $2^{31} x^{12}$.

An "arc piece" on a $Q$ can have the following punctures: a meridian to a third-level 2-handle; a meridian to a 2-handle and a meridian to a 1-handle in the fourth level, and, possibly, a meridian to each of the fifth-level 1-handles attached to the same fifth-level 2-handle. The number of the included $d_{6}$ 's (see Figure 38): $4(4 x)^{3}, 4(4 x)^{2}, 4 x$ and $2 x$, respectively. We approximate their sum by $2^{9} x^{3}$. There are at most $2^{7} n$ "arc pieces", so the number of the $E$ 's over a loop on a $Q$ is less than $2^{34} x^{10}$.

For the number of $E$ 's over any loop we will take $2^{34} x^{12}$.

2.4.3. Number of kinks on $E$ 's. Each $E$ consists of a $\tau_{5}$ surgered by two copies of a $\tau_{4}$. Then, each of the $\tau_{4}$ 's is turned into a contracted capped grope with the cap being an $R$ with $16 Q$ 's. So the self-intersection number of a cap is $1 / 4$ of the self-intersection number of a $D_{6}$ (see section 2.3.3).

The number of sixth-level core discs over a second-level 1-handle is $2 x_{4} 2 x_{5}^{\prime} 2 x_{6}^{\prime}$, which is smaller than $x_{6}^{\prime 2}$. The number of loops over a secondlevel 1-handle is then smaller than $x_{6}^{\prime 3}$.

The number of the $E$ 's is smaller than $2^{34} x^{12} x_{6}^{\prime 3}$ and we have to contract twice the number of the contracted capped gropes that were produced from the $\tau_{4}$ 's.

Using formulae (5) and (10), we have

$$
\pitchfork E \leq\left((17 z / 4)^{\left.2^{\left(2 \cdot 2^{34}\right.} x^{12} x_{6}{ }^{33}\right)},-\right) \text {. }
$$


2.4.4. Number of kinks on $D_{5}$ 's. Each $D_{5}$ has kinks on its four copies of a $P_{3}$ and on $4(4 x)^{3}$ copies of $d_{6}$ 's. The intersections between the different $D_{5}$ 's are those coming from the copies of the same $P_{3}$ 's and $d_{6}$ 's, and are removed by the finger moves. Each cap of an $D_{5}$ contains a copy of a $P_{3}$ and $(4 x)^{3}$ copies of $d_{6}$ 's. So the $x$ for formula (5) is: $2^{17} x^{7}+1$.

For the (less than) $x_{6}^{\prime 3}$ loops we approximate the number of $D_{5}$ 's over a second-level 1-handle with

$$
x_{6}^{\prime 3} \cdot x^{15} 10^{10^{3000 x}}
$$

Formula (5) then gives

$$
\text { in } D_{s} \leq\left(\left(17 \cdot 2^{18} x^{7}\right)^{\left.2^{x_{6}}{ }^{13} x^{15} 10^{10^{3000 x^{7}}},-\right)}\right.
$$

2.4.5. The $x_{7}^{\prime}$. From the previous sections we have the estimate:

$$
\begin{aligned}
& x_{6}^{\prime 3}\left(2^{34} x^{12}\left(17 \frac{z}{4}\right)^{\left.2^{\left(2 \cdot 2^{34}\right.} x^{12} x_{6}{ }^{13}\right)}\right. \\
& \left.+x^{15} 10^{10^{3000 x^{7}}}\left(17 \cdot 2^{18} x^{7}\right)^{\left.2^{x_{6}}{ }^{13} x^{15} 10^{10^{3000 x}}\right)}\right)
\end{aligned}
$$

We approximate this number by

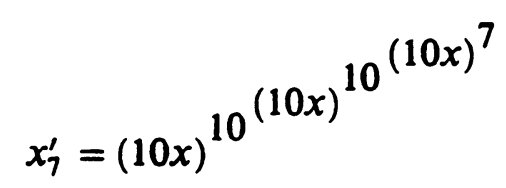

2.5. Proof of Theorem A. For the 'input tower' $T_{6}^{0}$ with the self-intersection numbers of the dises in $C_{2-6}^{0}$ equal to $(x, x)$ we have calculated that the embedded six-level Casson tower $T_{6}^{1}$ has the same self-intersection numbers through first three levels as the $T_{6}^{0}$ and for higher levels we have

$$
x_{5}^{\prime}=(10 x)^{2} \text {, }
$$

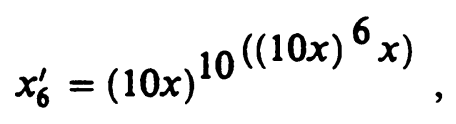

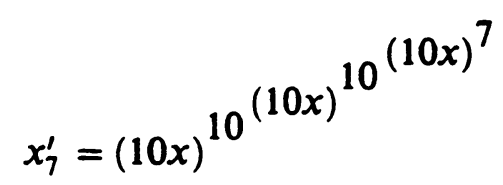

We apply the reimbedding algorithm on each six-level branch of the tower $T_{7}^{1}$ to obtain, in each of them, an embedded seven-level Casson tower. Because all the branches of $T_{7}^{1}$ have the same self-intersection numbers, the new towers will all look the same, i.e., they will have the same self-intersection numbers. Connecting a small neighborhood of the first level in $T_{7}^{1}$ with the embedded seven-level towers in the branches of $T_{7}^{1}$, we obtain an embedded eight-level 
Casson tower $T_{8}^{2}$ in $T_{7}^{1}$, again specified by a sequence of (nine) nonnegative numbers.

Continuing this process, we obtain a nested sequence of towers $\left\{T_{k+6}^{k}\right\}_{k=0}^{\infty}$ and, as it limit, a Casson handle $C H \hookrightarrow T_{6}^{0}$. Because our algorithm applied on a six-level tower does not change the first four levels of the tower, for $n \geq 5$, the number $y_{n}$, i.e., the number of the positive and negative kinks of the discs of the $n$th level of $C H$, is equal to the one of the tower $T_{i+6}^{i}$, for $i \geq n-3$. Consequently, to calculate $y_{n}$, we have to apply the algorithm $(n-3)$ times. After we do $(n-6)$ runs, we have the tower $T_{n}^{n-6}$ and the self-intersection number of its top level is calculated by the formula (c) with the $y_{n-1}$ as $x$. Then the result is plugged into formula (b) as the tower $T_{n+1}^{n-5}$ is constructed. Finally, we have to apply to this result formula (a) and we can take the resulting number to be $y_{n}$.

After applying the algorithm once on $T_{6}^{0}$, we have the conclusions (1) and (2) of the theorem satisfied.

After the second application, we have $y_{6}$, which is $x_{6}^{\prime}$ substituted in formula (a):

$$
\left(10(10 x)^{10^{\left((10 x)^{6} x\right)}}\right)^{2},
$$

and we will take for $y_{6}$ a larger number

$$
y_{6}=(10 x)^{10^{(10 x)^{10}}} \text {. }
$$

For $n>6$ we will use induction to prove statement (4) of the theorem. To obtain $y_{n}$ (for $n>6$ ) we need to substitute $y_{n-1}$ into (c), then the result into (b) and finally apply (a). (Note that we should substitute the $(n-1)$ st intersection number of $T_{n-1}^{n-7}$ instead of $y_{n-1}$, but $y_{n-1}$ is bigger than this number.) If we denote by $x_{n}^{\prime}$ the $n$th intersection number of $T_{n}^{n-6}$, and by $x_{n}^{\prime \prime}$ the $n$th intersection number of $T_{n+1}^{n-5}$, then

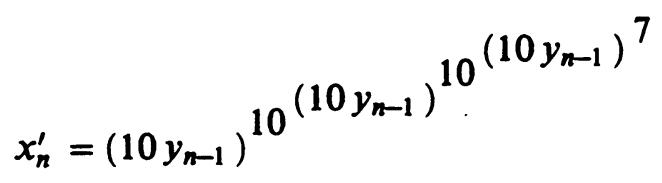

and

$$
x_{n}^{\prime \prime}=\left(10 x_{n-1}^{\prime}\right)^{\left.10^{\left(\left(10 x_{n-1}^{\prime}\right)^{6}\right.} x_{n-1}^{\prime}\right)},
$$

so after slinstituting this number in (a), the number is less than

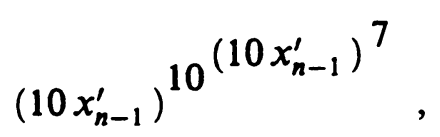

which is equal to 
(d)
$\left(10\left(10 y_{n-1}\right)^{10} 0^{\left(10 y_{n-1}\right)}\right.$

Adding the different exponents together and substituting the bigger one into the smaller ones, it can be easily seen that the number (d) is smaller than

(e)

$$
\left(10 y_{n-1}\right)^{10}\left(10 y_{n-1}\right)^{10^{\left(10 y_{n-1}\right)^{10}}}{ }^{\left(10 y_{n-1}\right)^{8}}
$$

For each $n>6, y_{n-1}$ itself looks like the stack of powers $(10 x)^{10 W}$, where

$$
\left.W=(10 x)^{\left(10^{\circ}\right)}\right) .
$$

The number in (e) looks like

$\left(10(10 x)^{\left(10^{W}\right)}\right),\left(10^{\left(10(10 x)^{\left(10^{W}\right.}\right)}\right)\left(^{\left.\left(10^{\left(10(10 x)^{\left(10^{W}\right.}\right)}\right)^{\left(10^{\left(10 y_{n-1}\right)^{8}}\right)}\right)}\right)$

Because "the stacks of powers" are all of the same length and the formula should be evaluated from right to left, using inequalities like

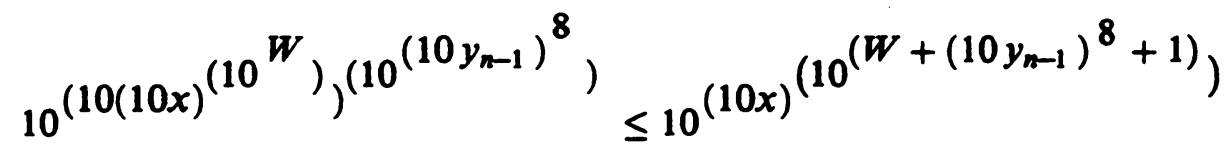

it is easy to see that this number is smaller than

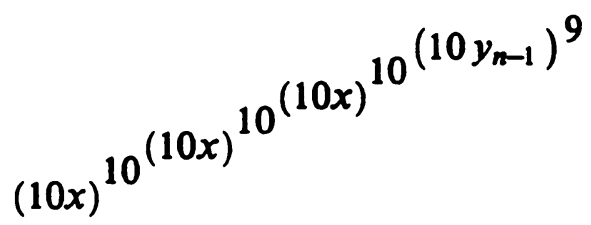

For the two topmost exponents we have the inequality

$$
\left(10 y_{n-1}\right)^{9}<(10 x)^{\left(10^{y_{n-1}}\right)}
$$

so we can take $y_{n}$ to be

$$
y_{n}=(10 x)^{10^{(10 x)^{10^{(10 x)}}}} 10^{(10 x)^{10^{y_{n-1}}}}
$$




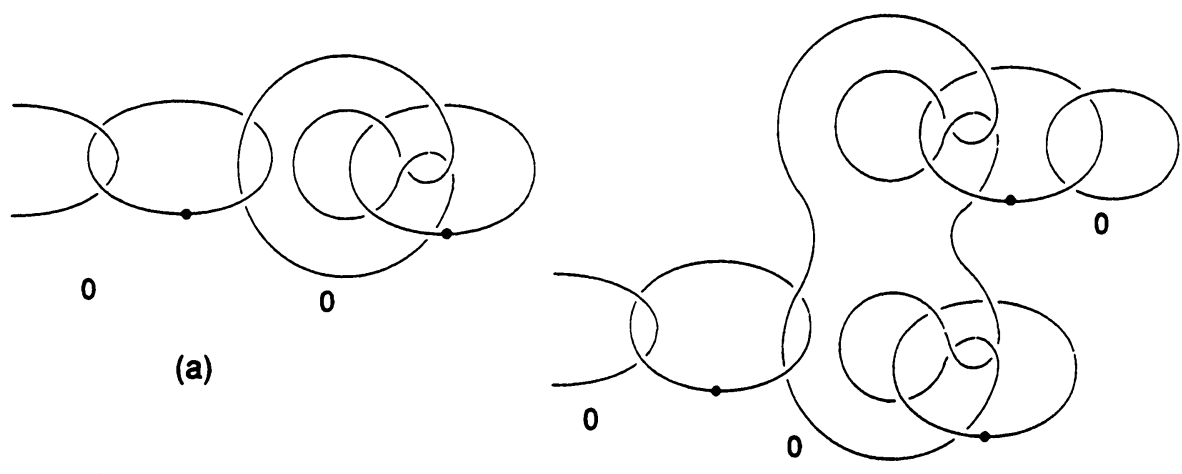

(b)

FIGURE 1. Figure (b) differs from (a) by a complementary pair of 1- and 2-handles.

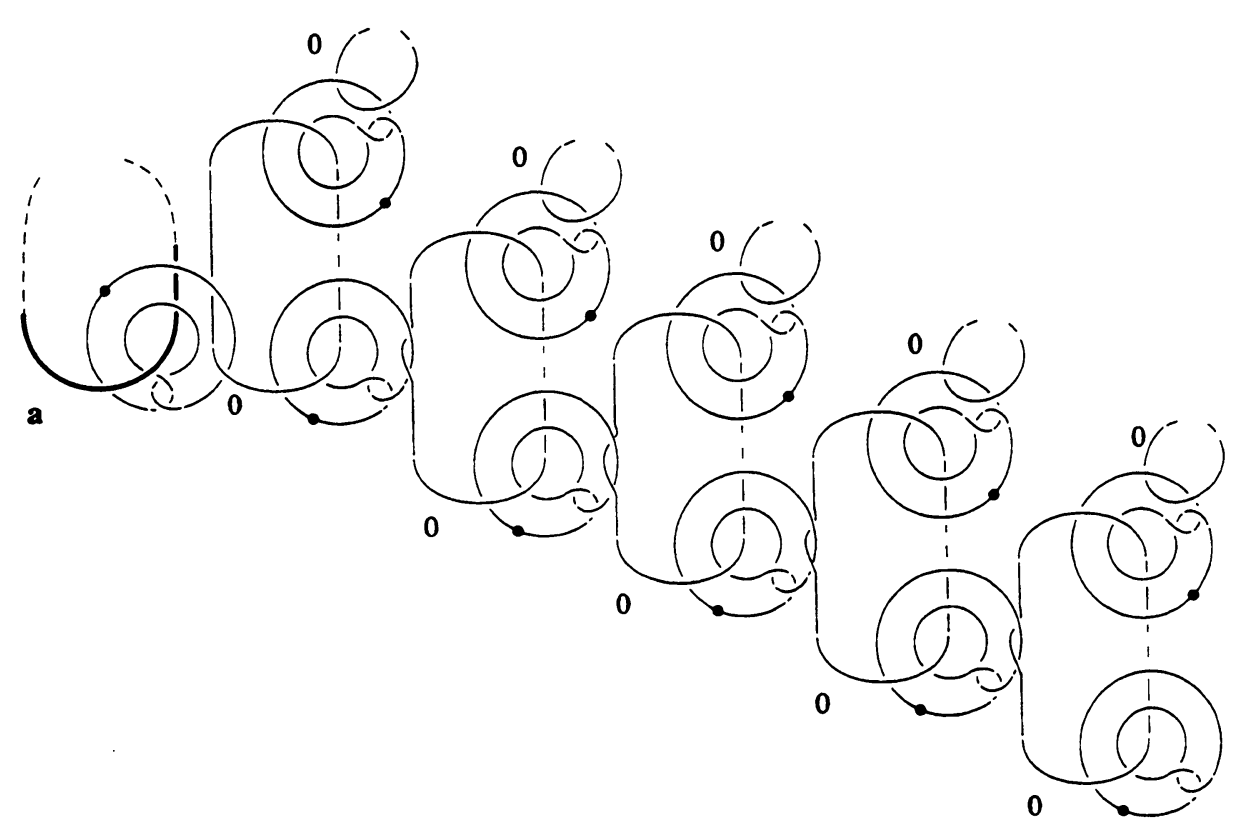

FIGURE 2. A single branch of a symmetrized "input" Casson tower is shown. For each of the 2-handles of the $n$th level there are $2 x_{n}$-many 1-handles attached to it, $x$-many for a positive kink and $x$-many with a negative kink. 


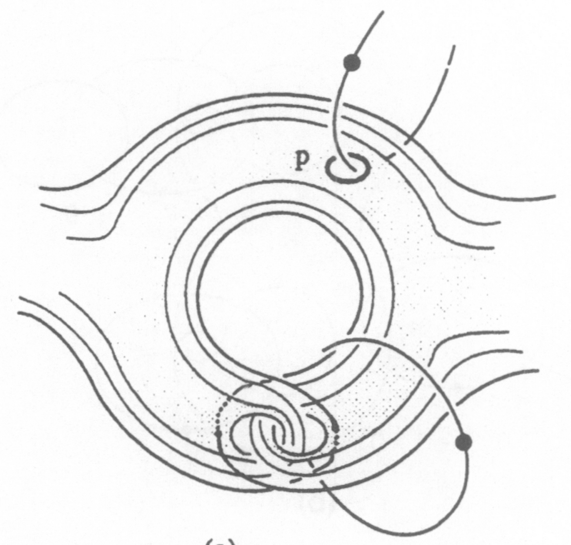

(a)

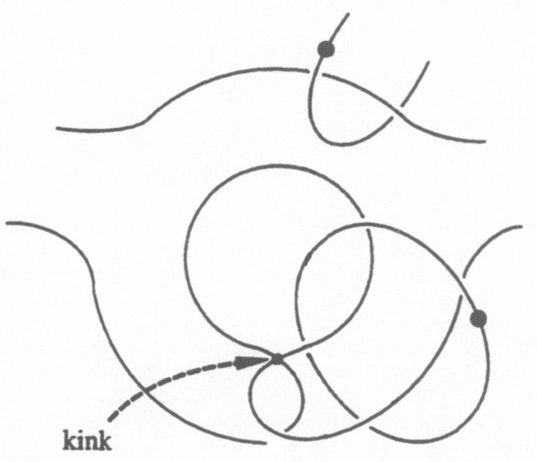

(c)

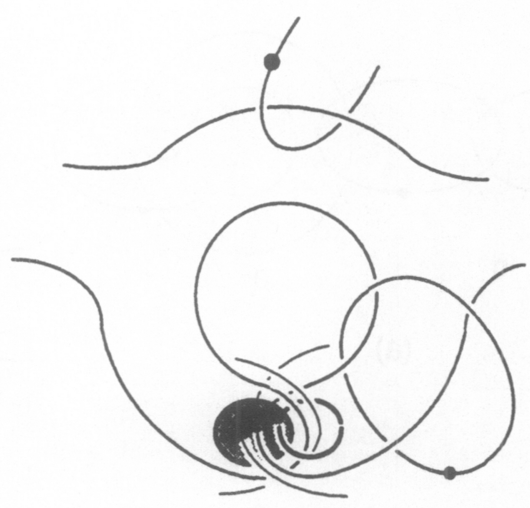

(b)

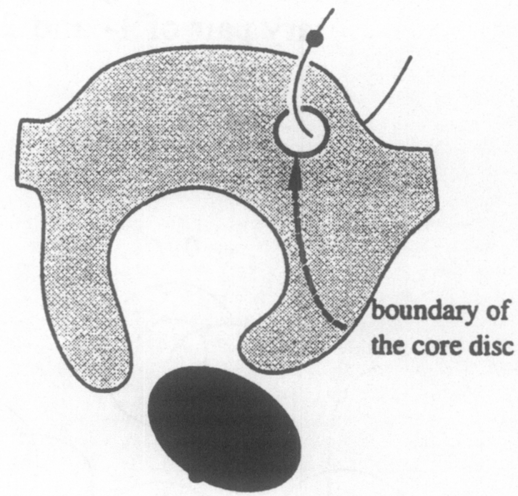

(d)

Figure 3. A core disc and its parallel copy.

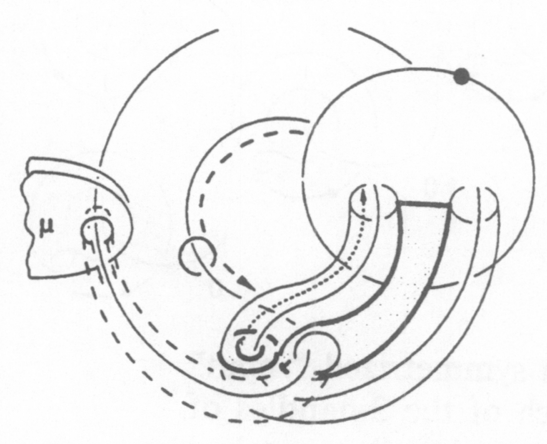

(a)

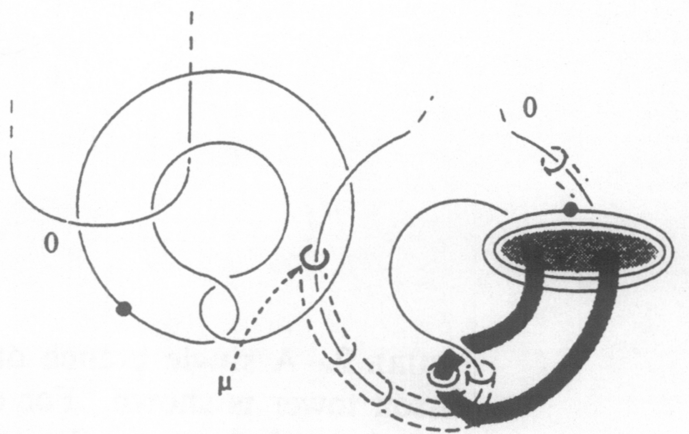

(b)

FIGURE 4. Part (a) depicts the two punctured caps of a characteristic torus. In part (b) a pipe connecting one of the caps with a 1-handle on the level below is visible. The shaded area is the horizontal part of the characteristic torus. 


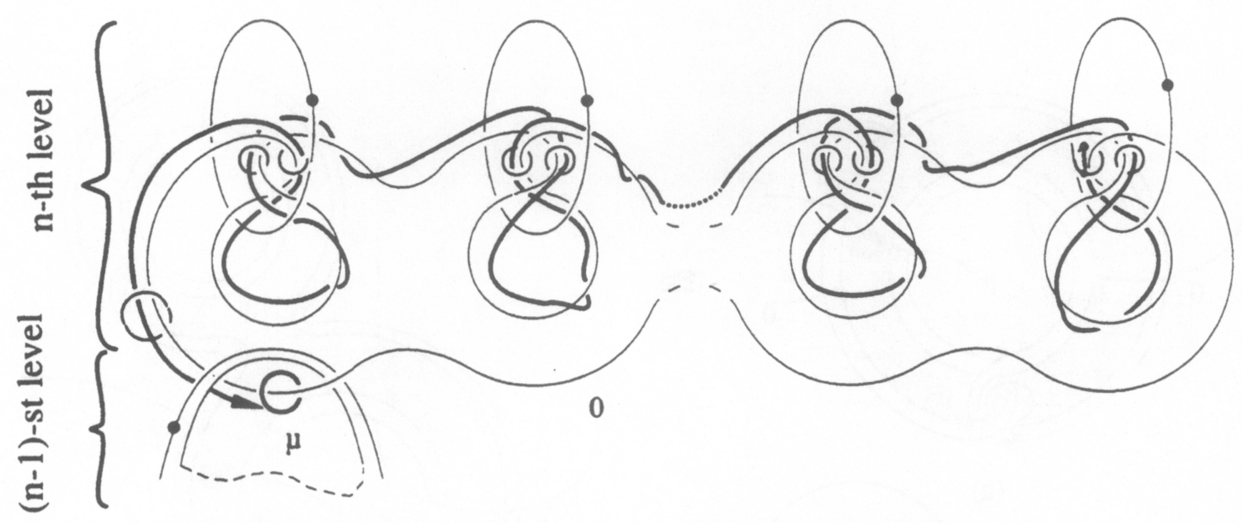

FIGURE 5. A "guiding path" on an $n$ th-level 2-handle.

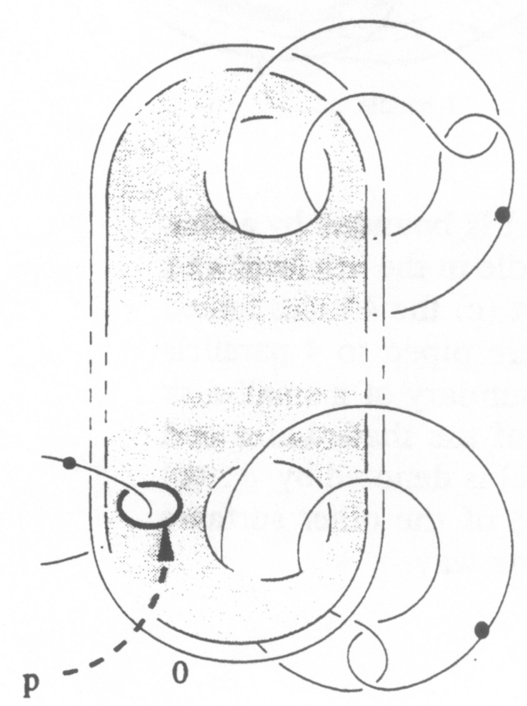

(a)

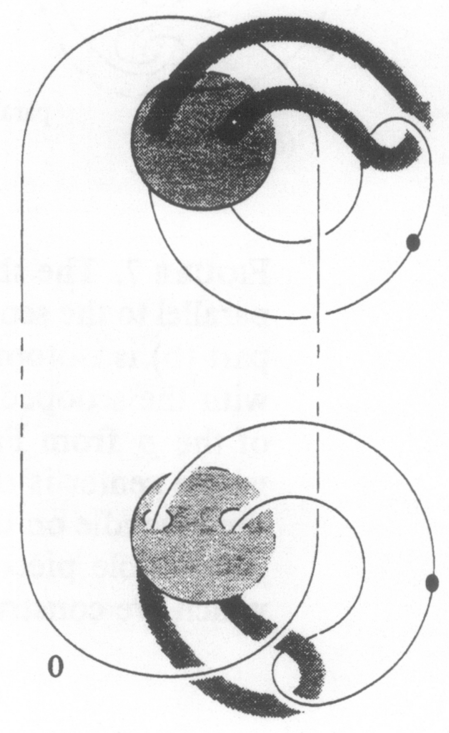

(b)

FIGURE 6. A single 'kinky handle' from Figure 2. The union of the shaded areas together with a parallel of the core of the 2-handle will be called $a$ core surface. The boundary of this surface is denoted by $p$. 


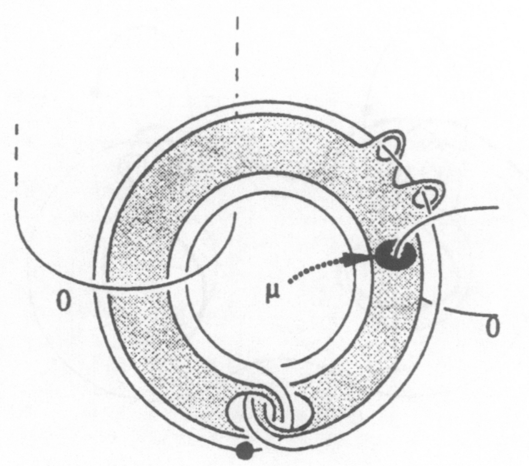

(a)

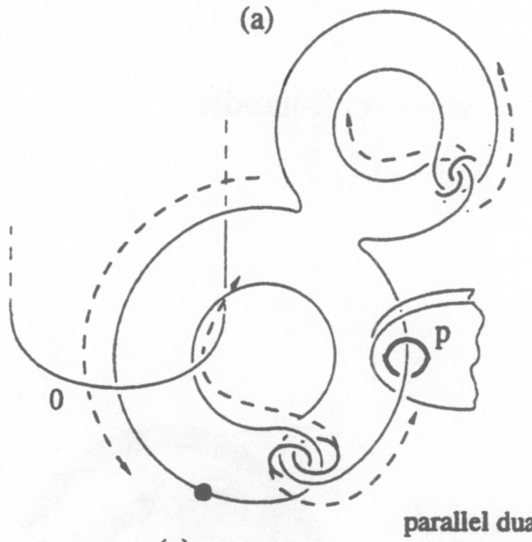

(c)

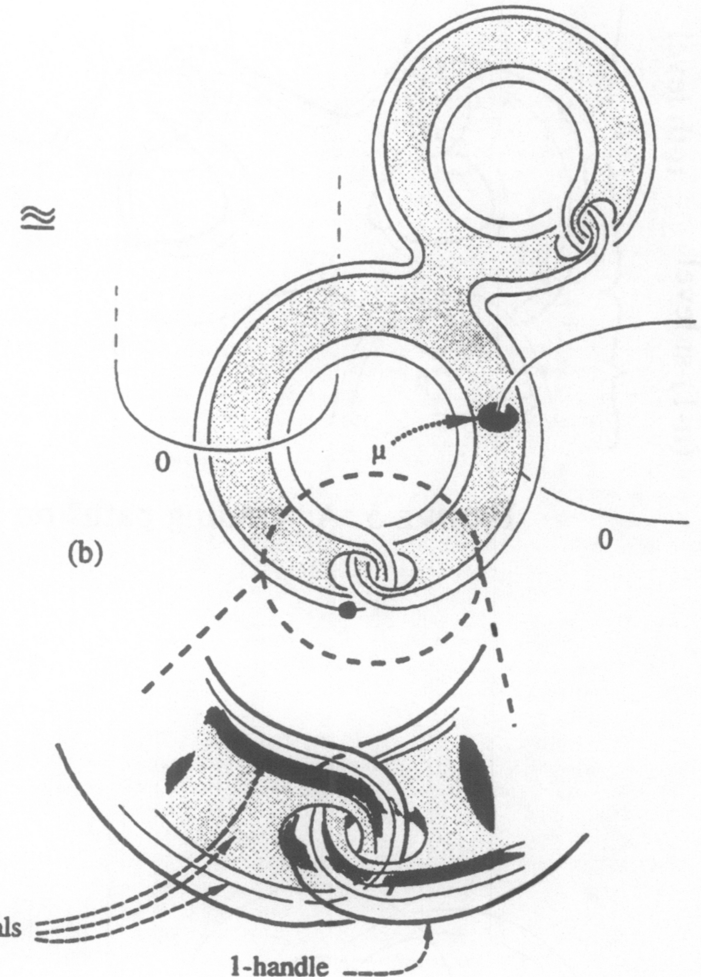

(d)

FIGURE 7. The shaded area in (a) is bounded by a disk parallel to the scooped-out 2-handle in the $n$th level and part (b) is isotopic to (a). In part (c) the 4 holes linked with the scooped-out 2-handle are piped to 4 parallels of the $p$ from Figure 3. The boundary of a small disk whose center is the intersection of the shaded area and the 2-handle on the $(n+1)$ st level is denoted by $\mu$. (d) The visible pieces in the picture of the other surfaces which are confstructed in the same way. 


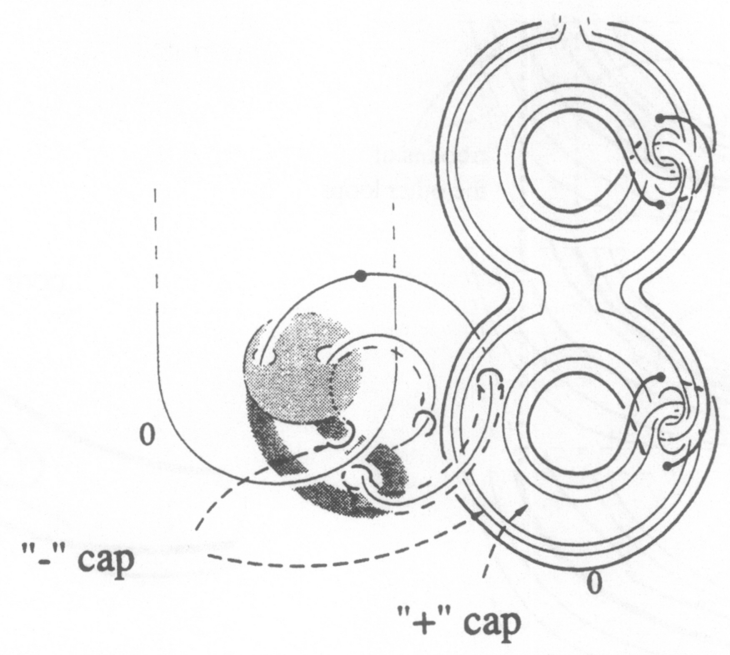

Figure 8. A capped grope in the $n$th level which is the result of the operation $\theta_{1}(a)$ applied to a punctured torus from Figure 6(b). The shaded area on the right is the horizontal part of a parallel of a core disc on the $(n+1)$ st level and it is now a piece of the + cap. The - cap is another parallel with its horizontal part in a different slice. 


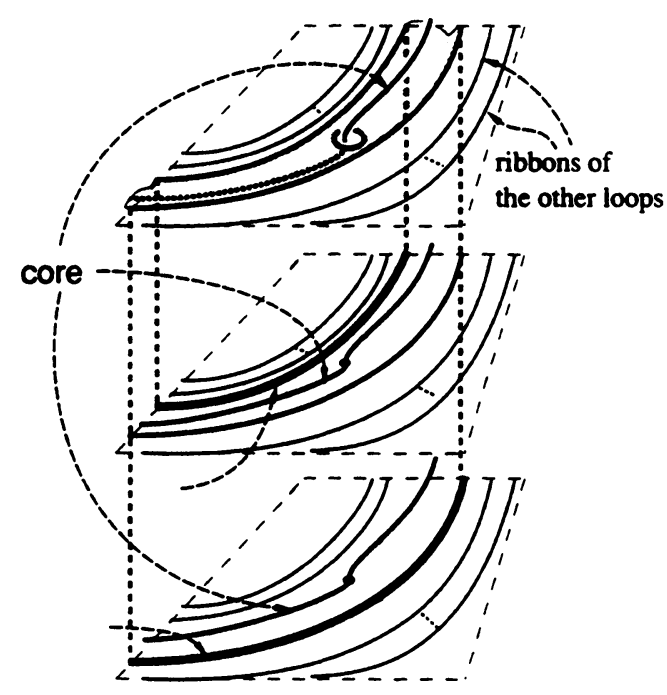

(a)

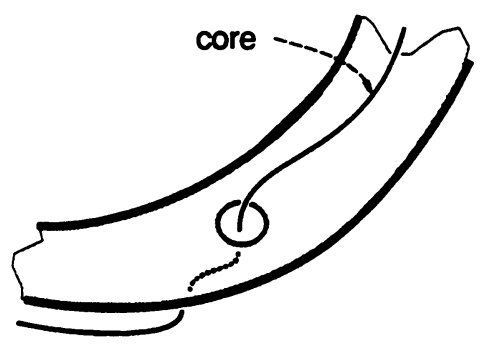

(b)

Figure 9. (a) Three different slices are shown, where, from the bottom up, the first one contains a piece of the arc denoted by $\mu$, the second contains the arc $\lambda$ and a parallel copy of $\mu$ and in the topmost one the copies of the both arcs bound a punctured ribbon. All the slices in between contain a parallel copy of $\mu$ and the ones between the second and third also contain a copy of $\lambda$. Consequently, in this part of the collar neighborhood the arcs $\lambda$ and $\mu$ bound a punctured ribbon. Also, parts of ribbons for the pairs of arcs parallel to $\lambda$ and $\mu$ are visible in each slice as the arcs or ribbons parallel to the ones in the topmost slice. It is assumed that no slice contains more than one ribbon. (b) The same punctured ribbon and arcs $\lambda$ and $\mu$, but compressed in a single slice. 


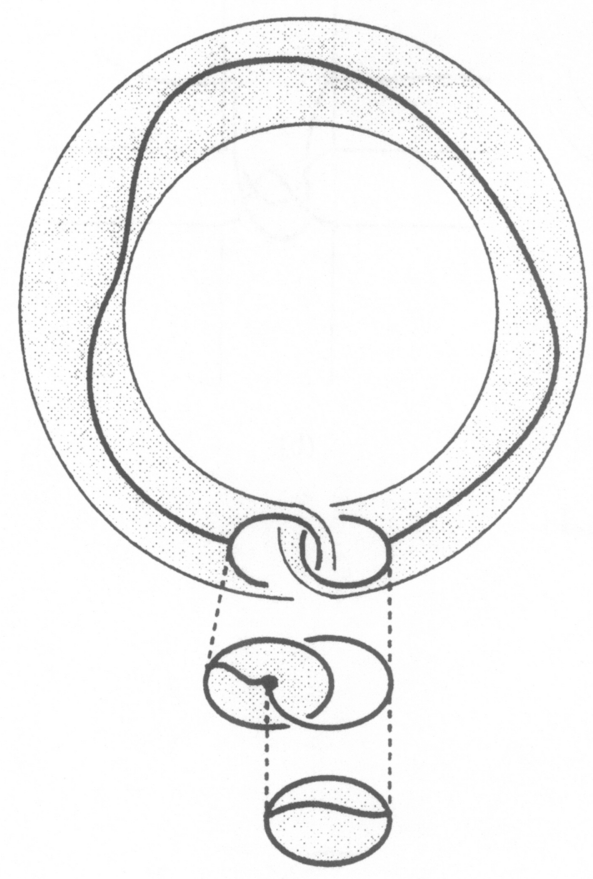

(a)

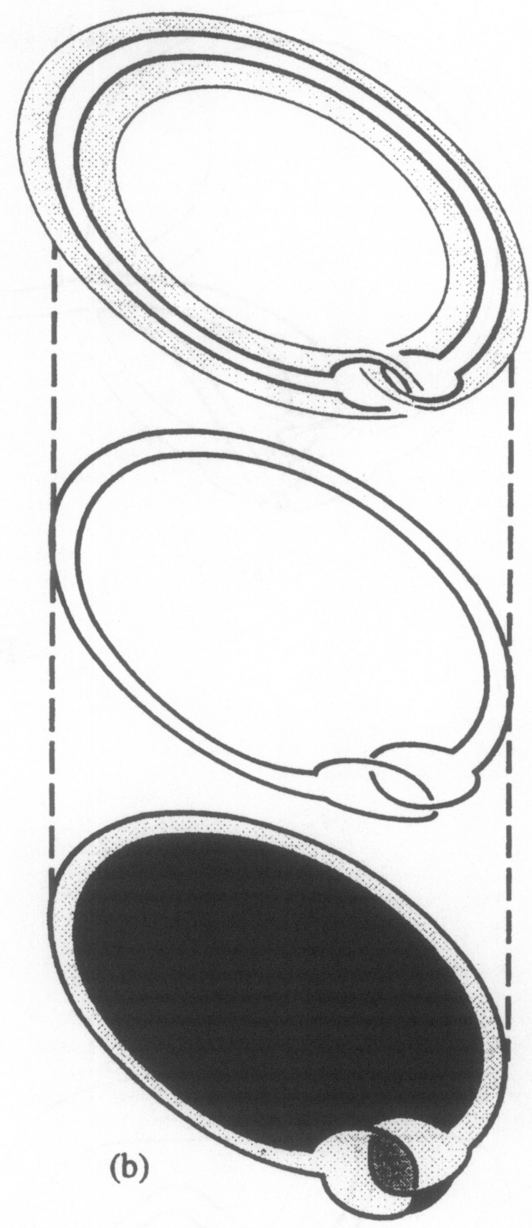

Figure 10. Capping of a standard loop for a positive kink. 


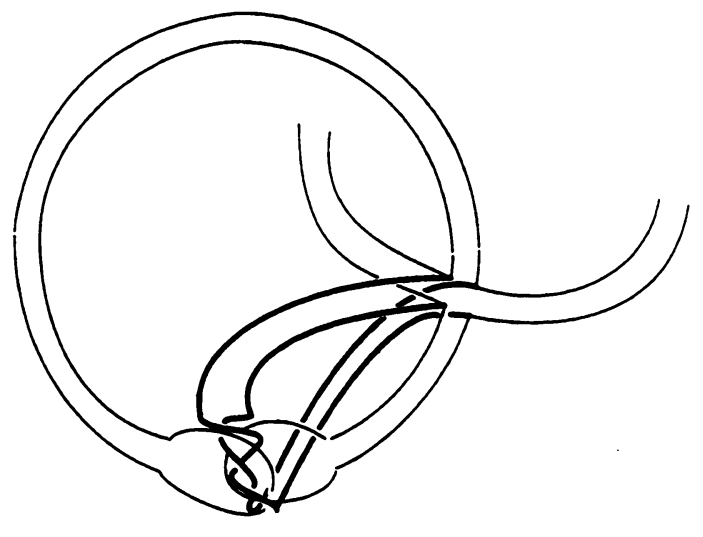

(a)

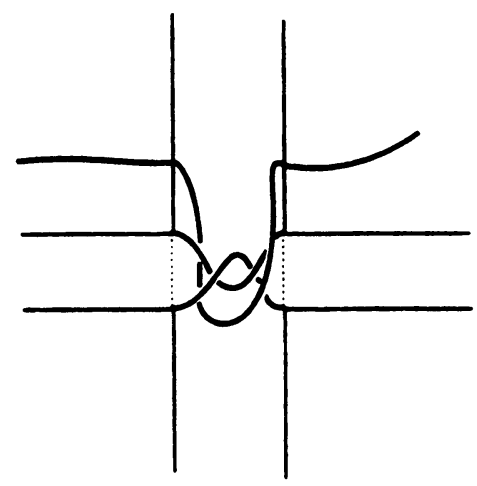

(b)

Figure 11

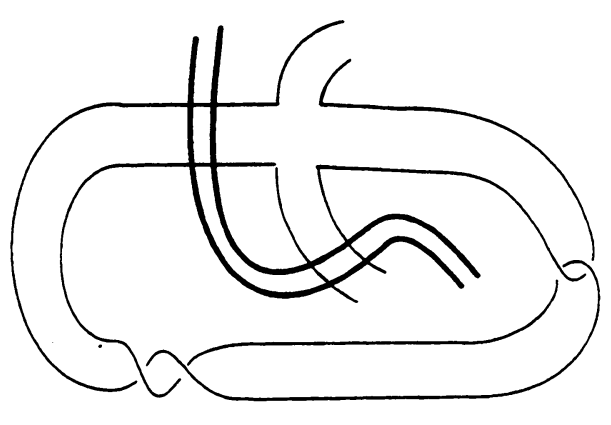

(a)

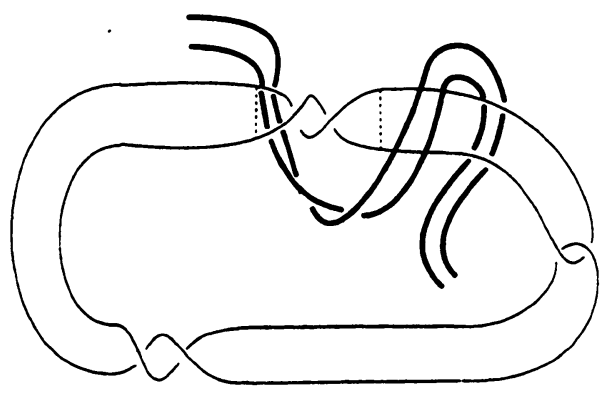

(c)

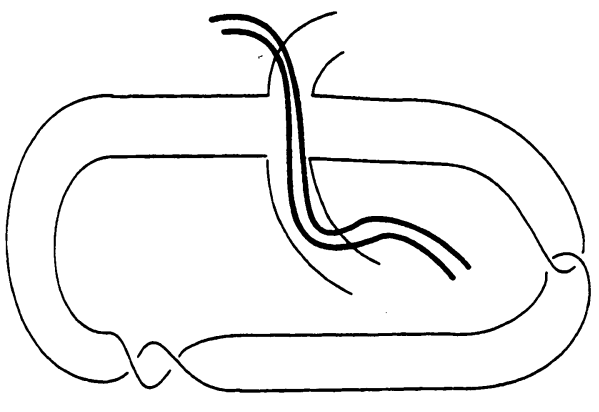

(b)

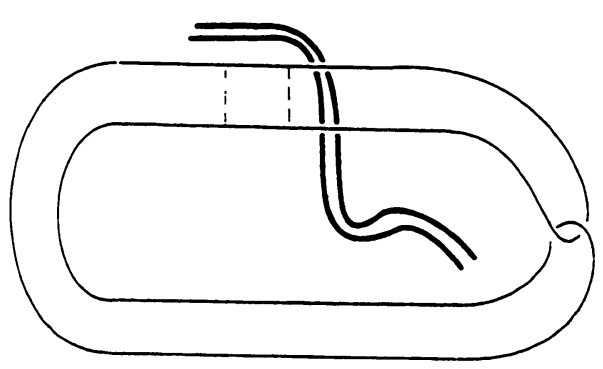

(d)

FIGURE 12 


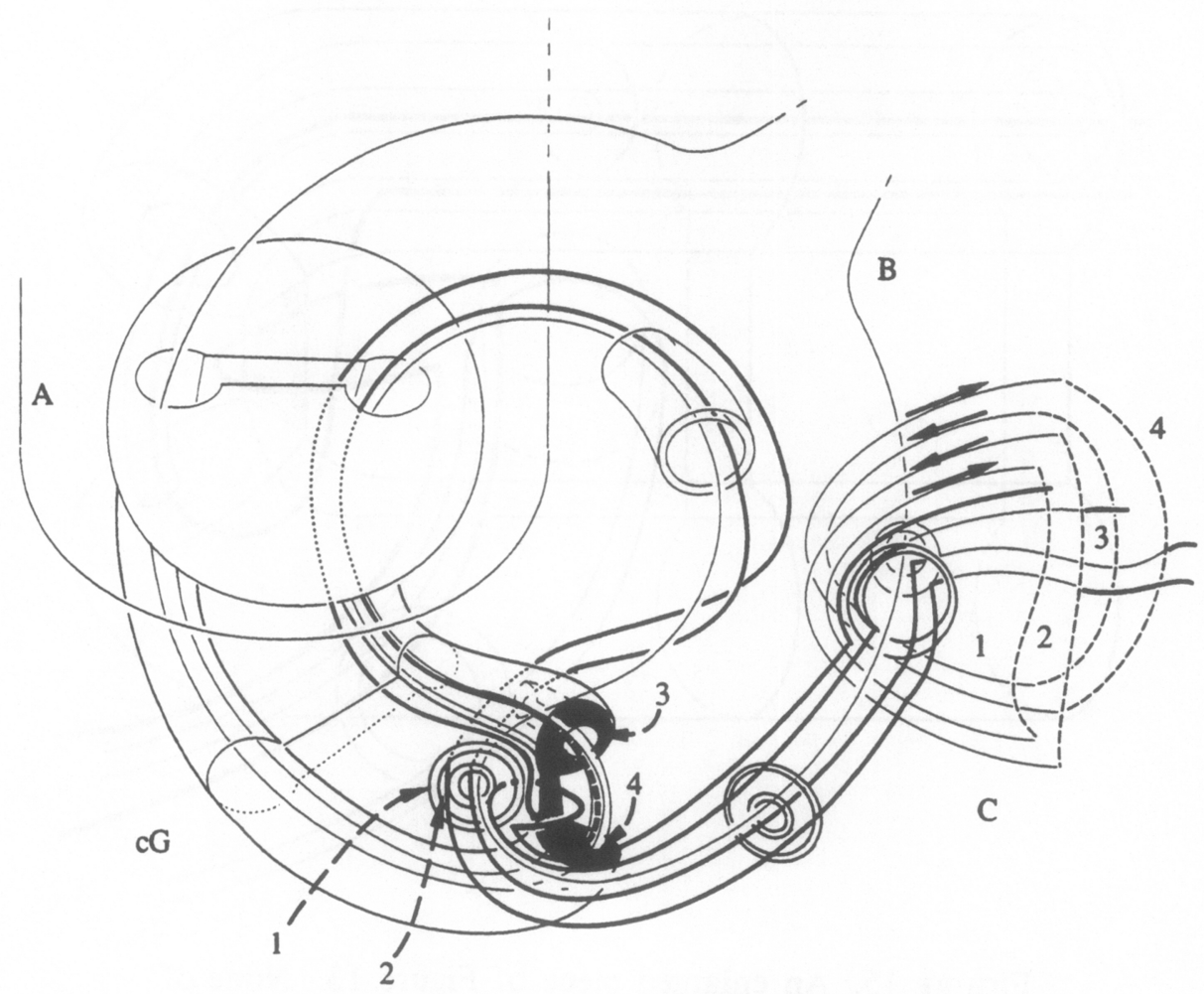

FIGURE 13

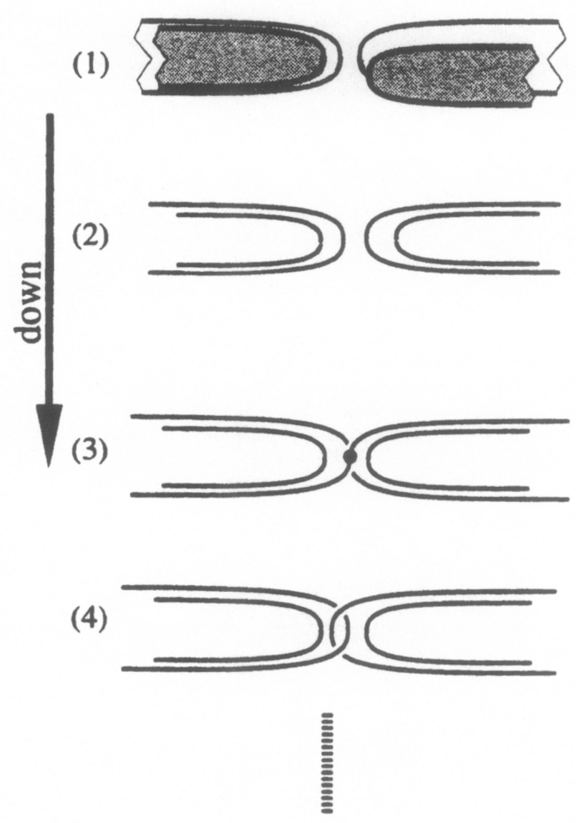

(5)

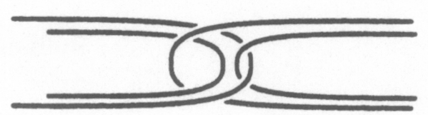

(6)
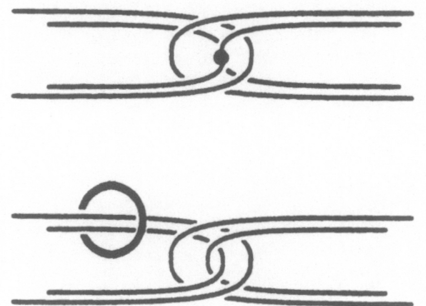

(7)

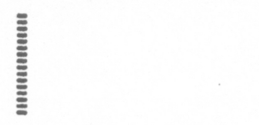

FIGURE 14 


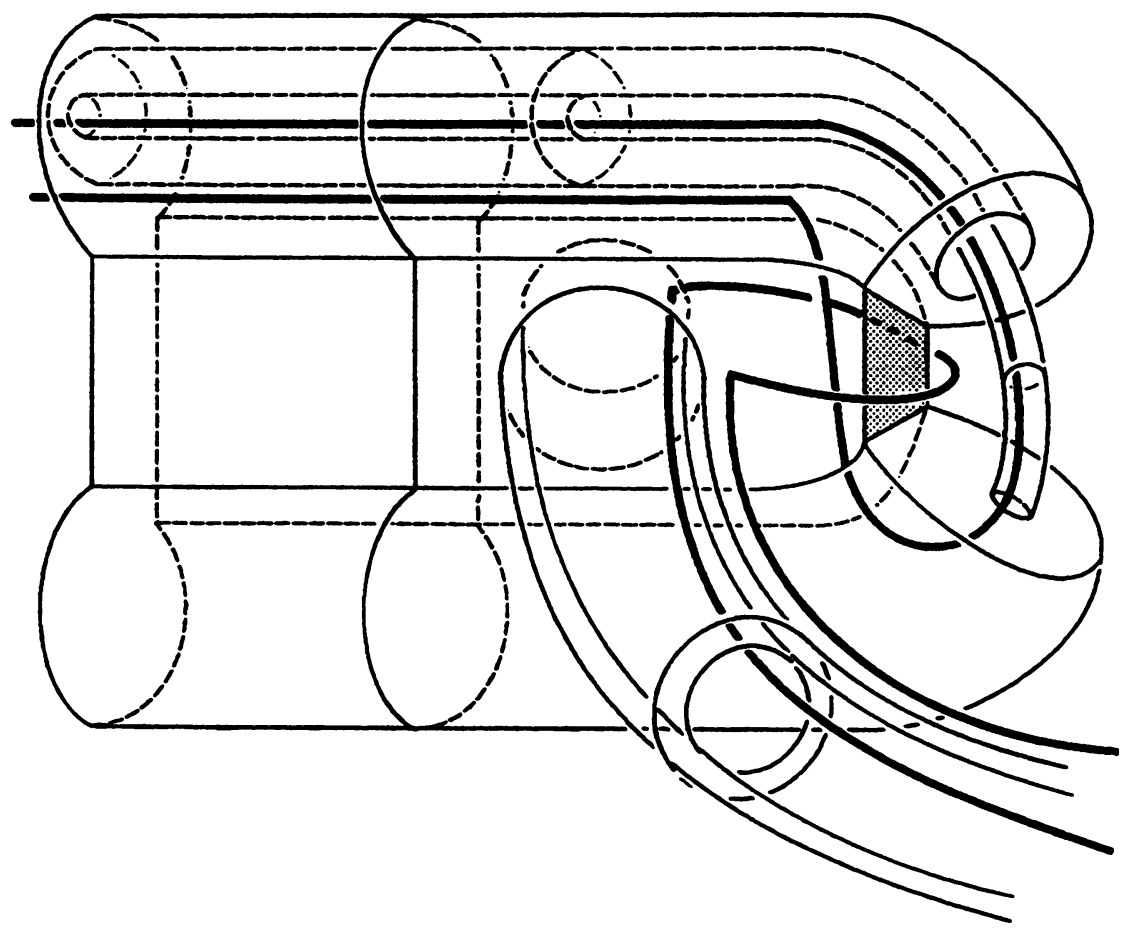

Figure 15. An enlarged piece of Figure 13. None of the pieces of the cores $A, B$ or $C$ is visible. 


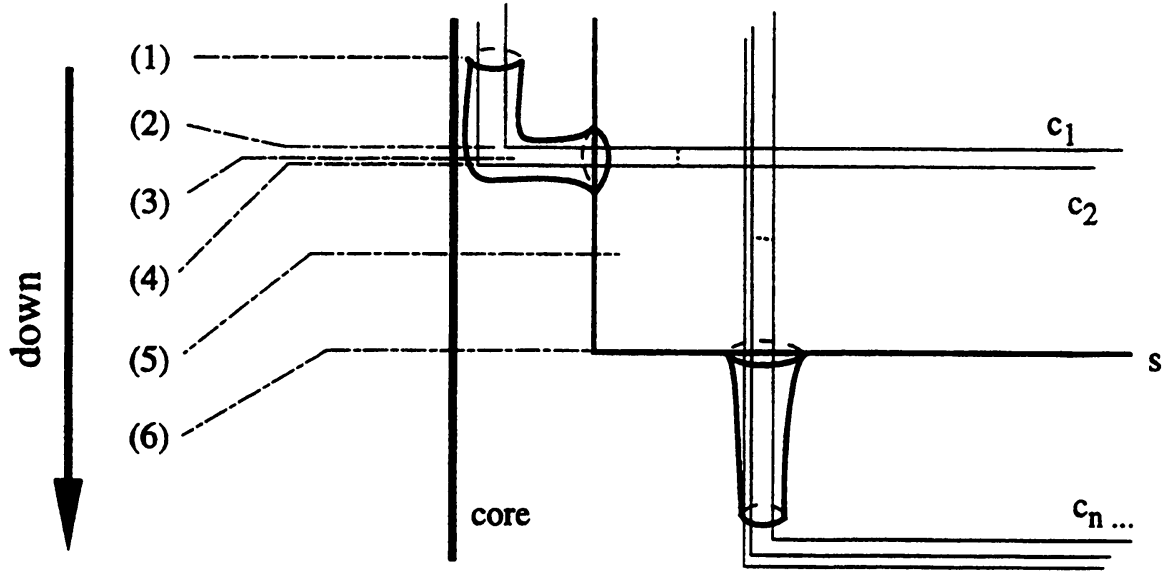

(1)

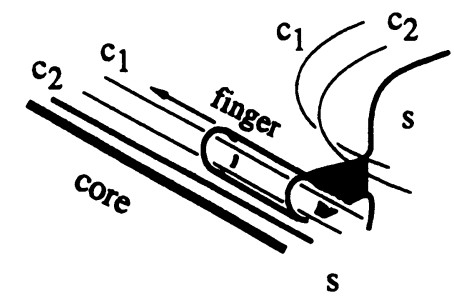

(2)

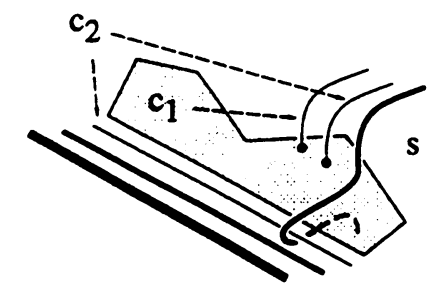

(4)

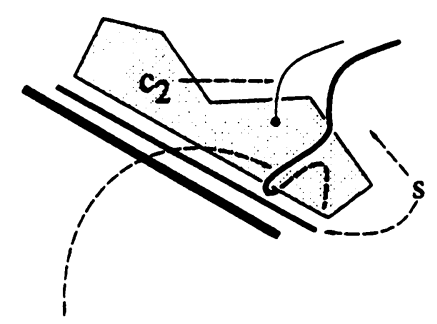

added finger

(5)

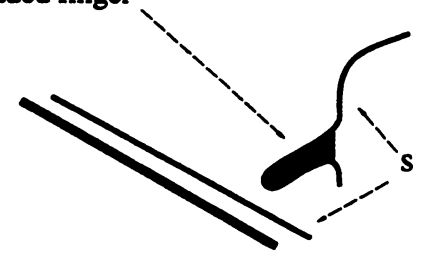

(3)

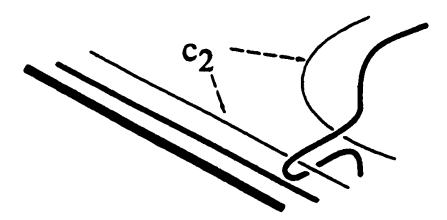

(6)

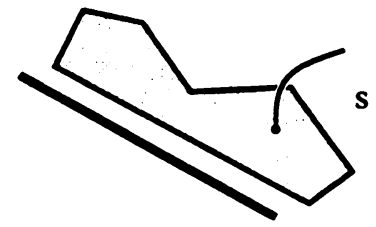

FIGURE 16 


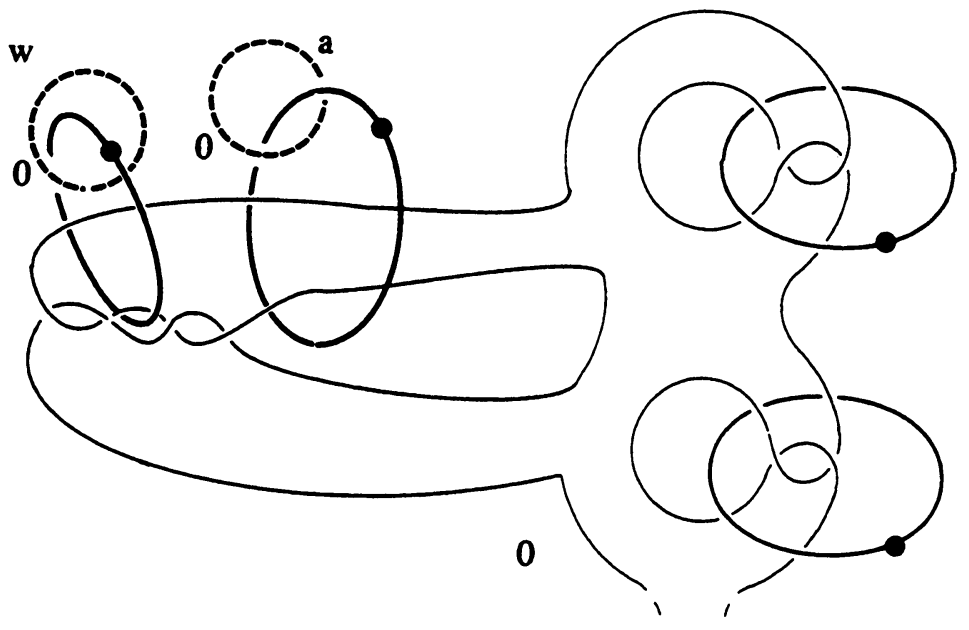

Figure 17

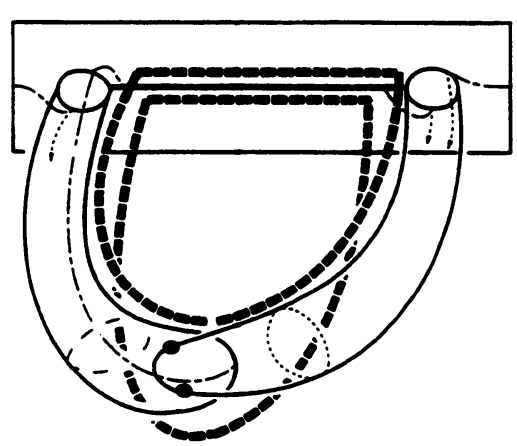

(a)

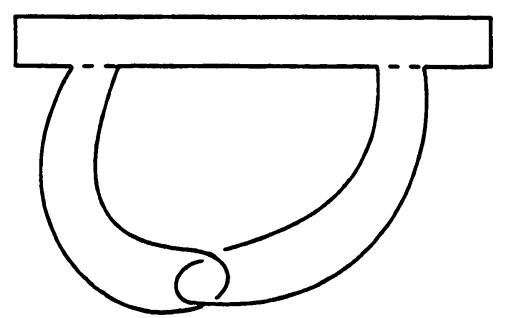

(c)

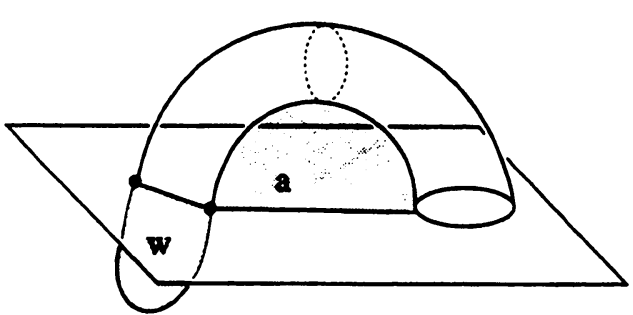

(b)

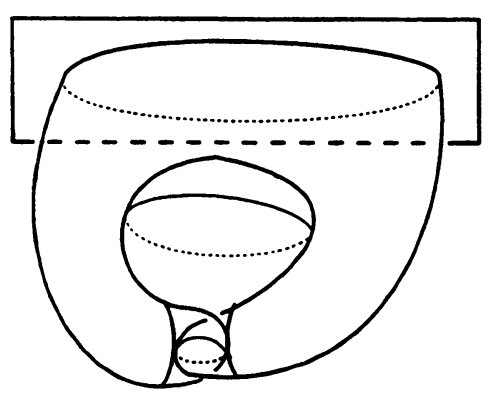

(d)

FIGURE 18 



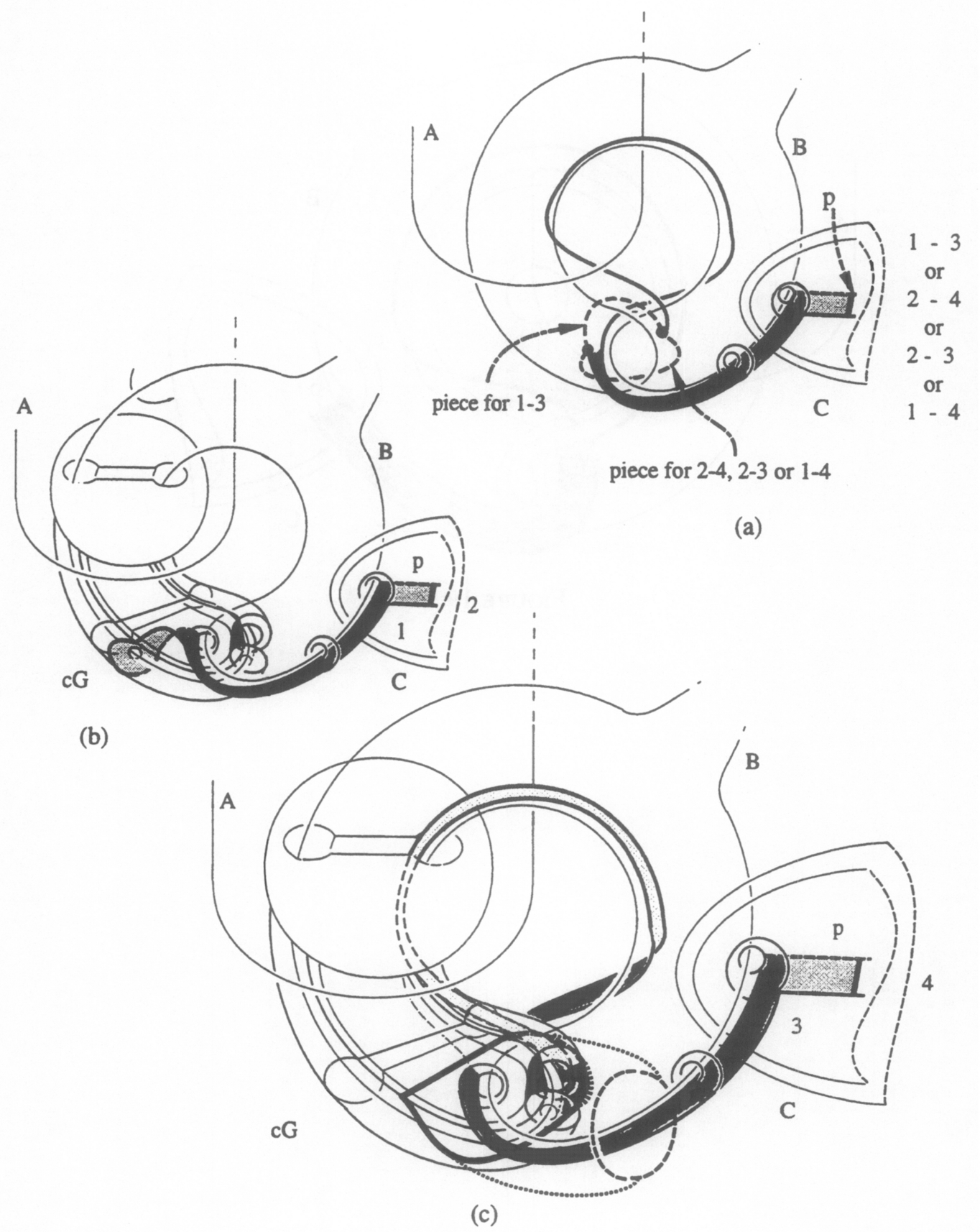

Figure 20 


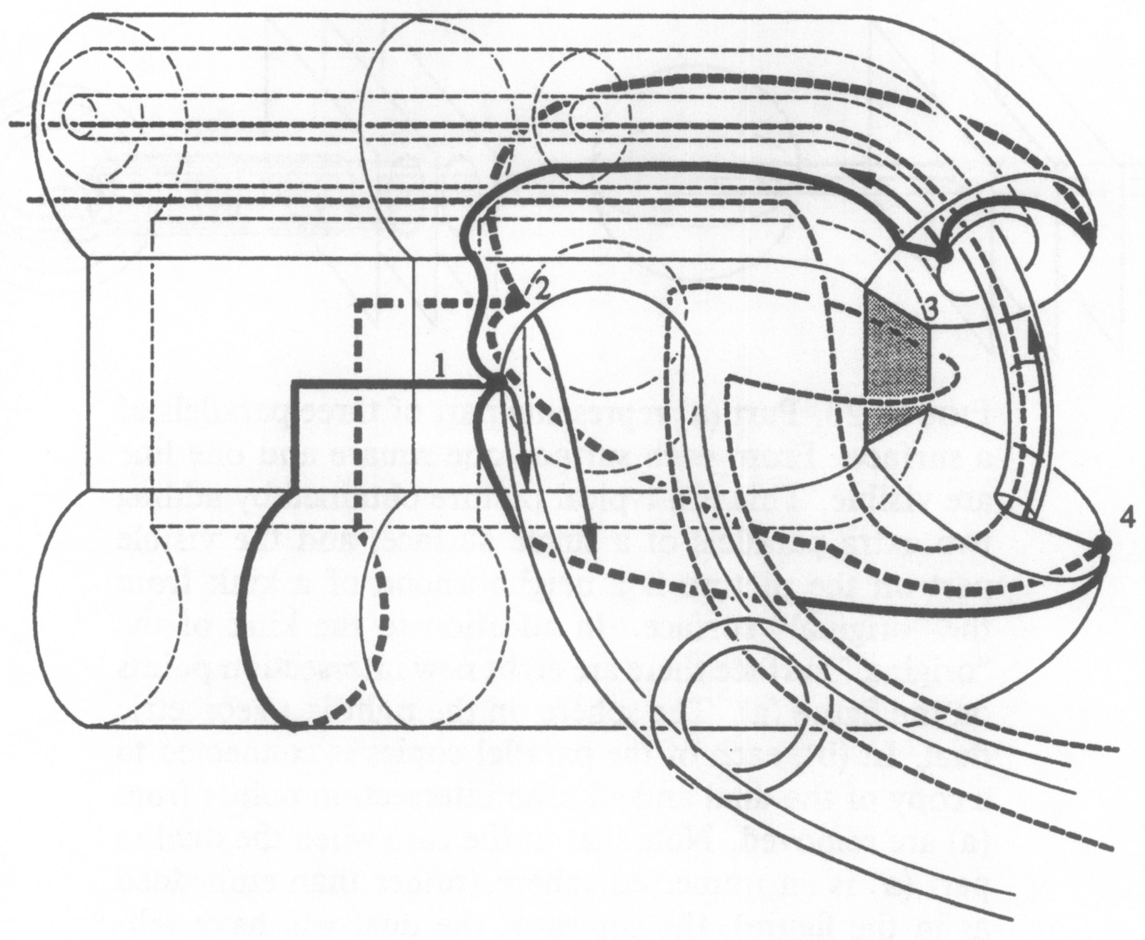

FIGURE 21

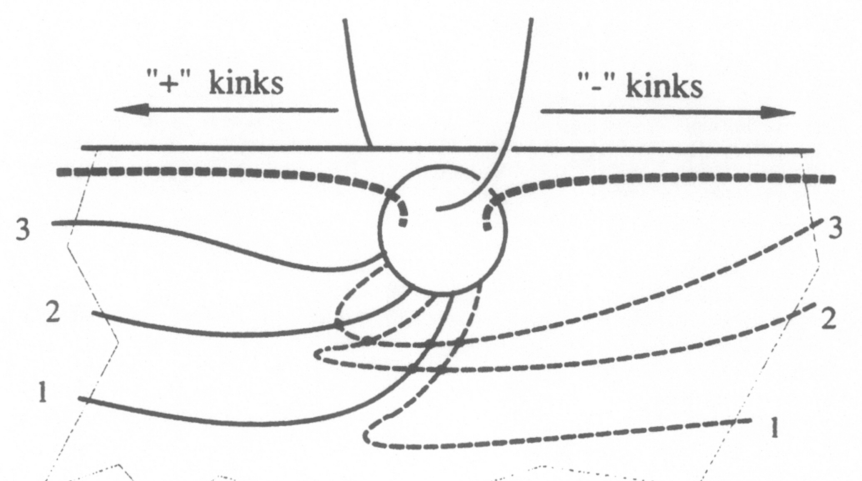

FIGURE 22 

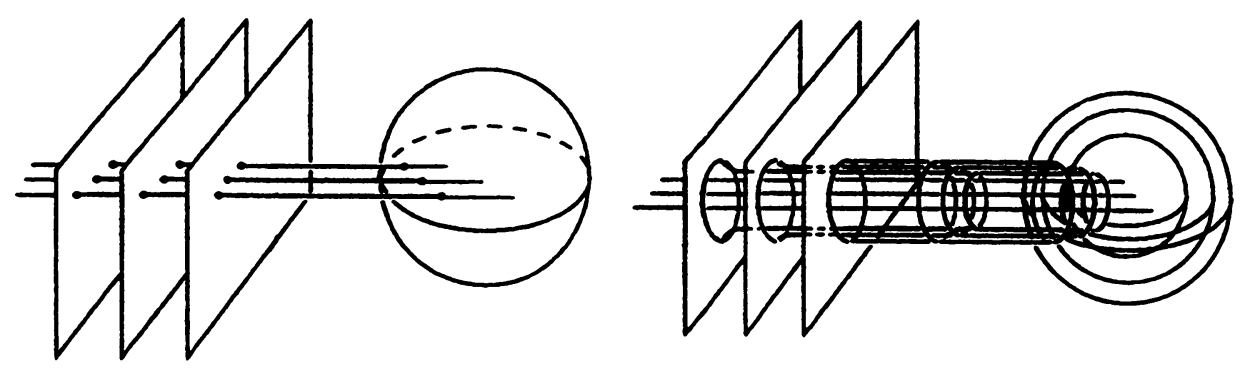

FIGURE 23. Part (a) represents part of three parallels of a surface. From each surface one square and one line are visible. This is a typical picture obtained by adding two extra parallels of a single surface, and the visible part on the picture is a neighborhood of a kink from the "original" surface. In addition to the kink of the "original" surface there are eight new intersection points on the figure (a). The sphere on the right is a geometric dual. In (b), each of the parallel copies is connected to a copy of the dual and all nine intersection points from (a) are removed. Note that in the case when the dual in part (a) is an immersed sphere (rather than embedded as in the figure), the copies of the dual will have selfintersections and intersections and the three surfaces in (b) are not disjoint. We will use this construction in a situation when the dual is an immersed sphere, but its copies are still disjoint immersed spheres. In this "singular" case the resulting surfaces in (b) have selfintersections, but are disjoint. 


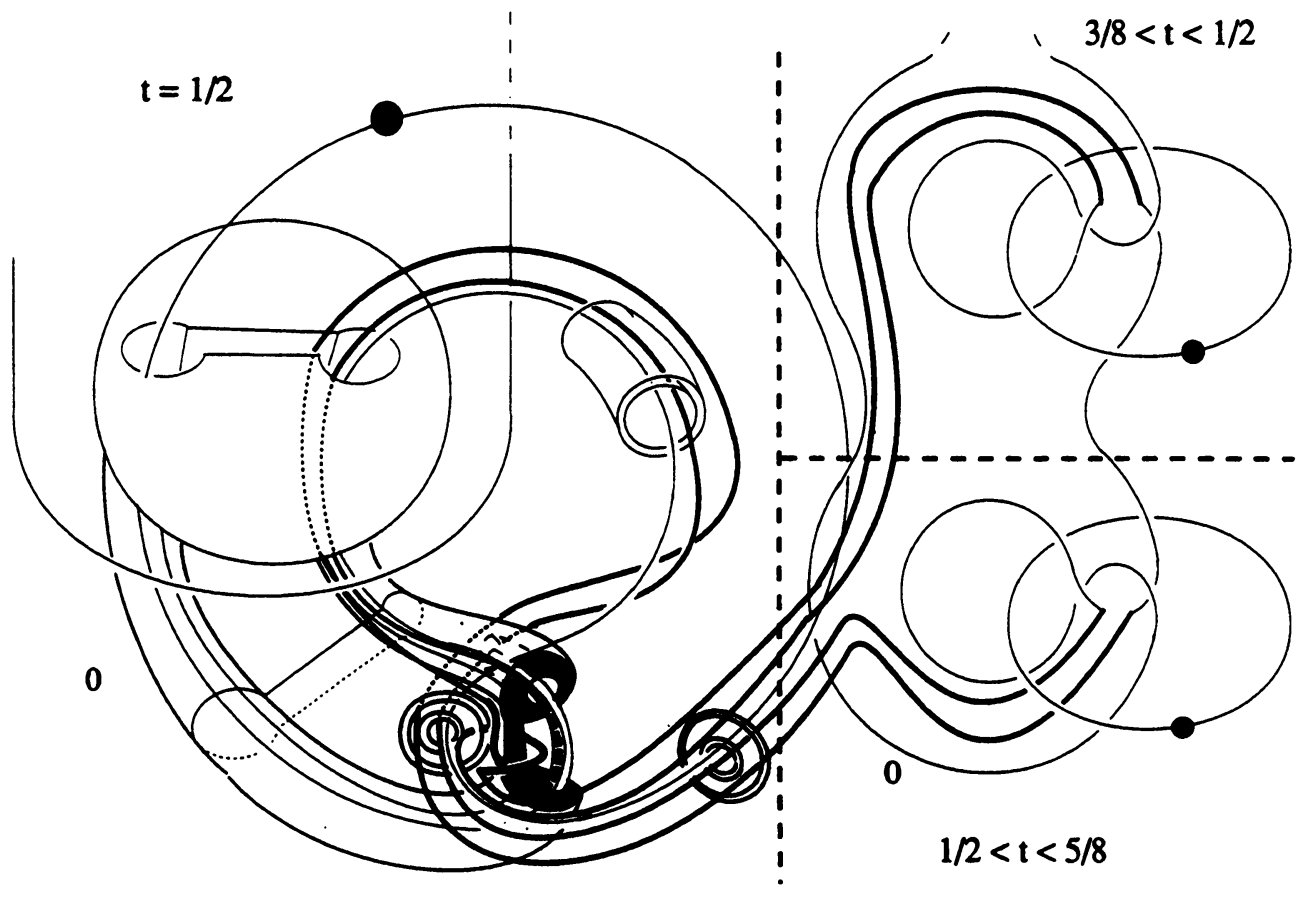

Figure 24 


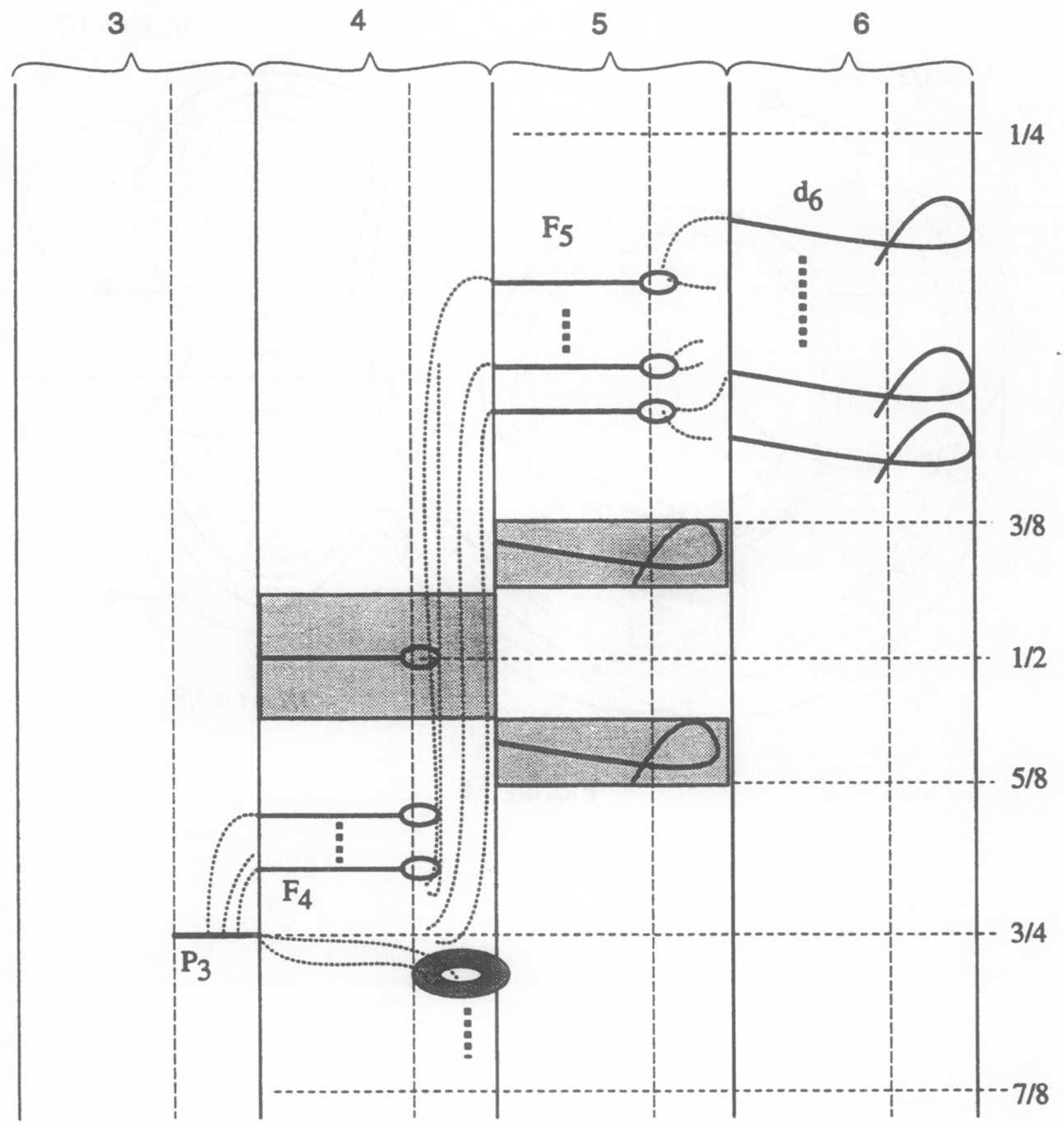

Figure 25. A fifth-level dual $D_{5}$. 


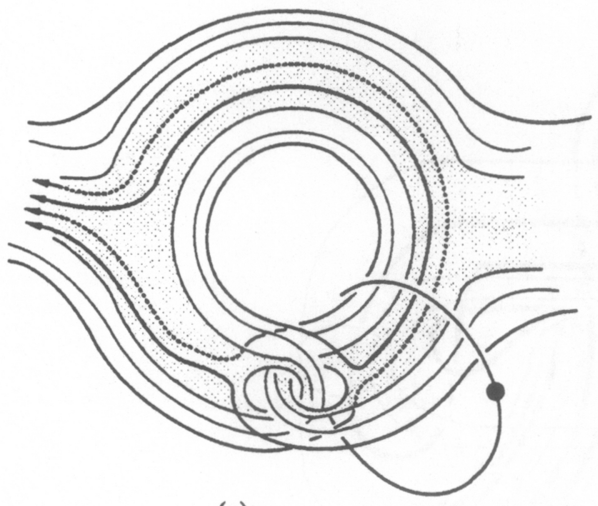

(a)

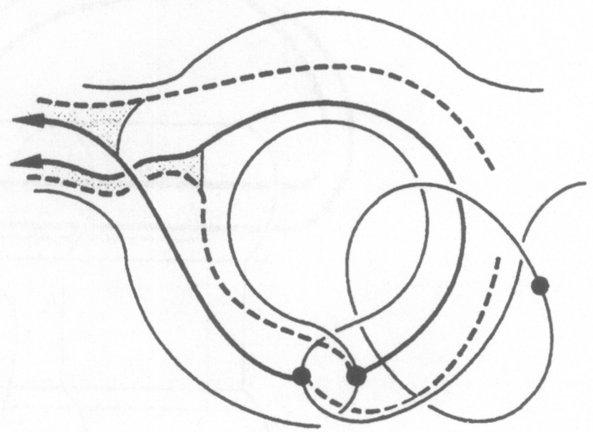

(b)

FIGURE 26

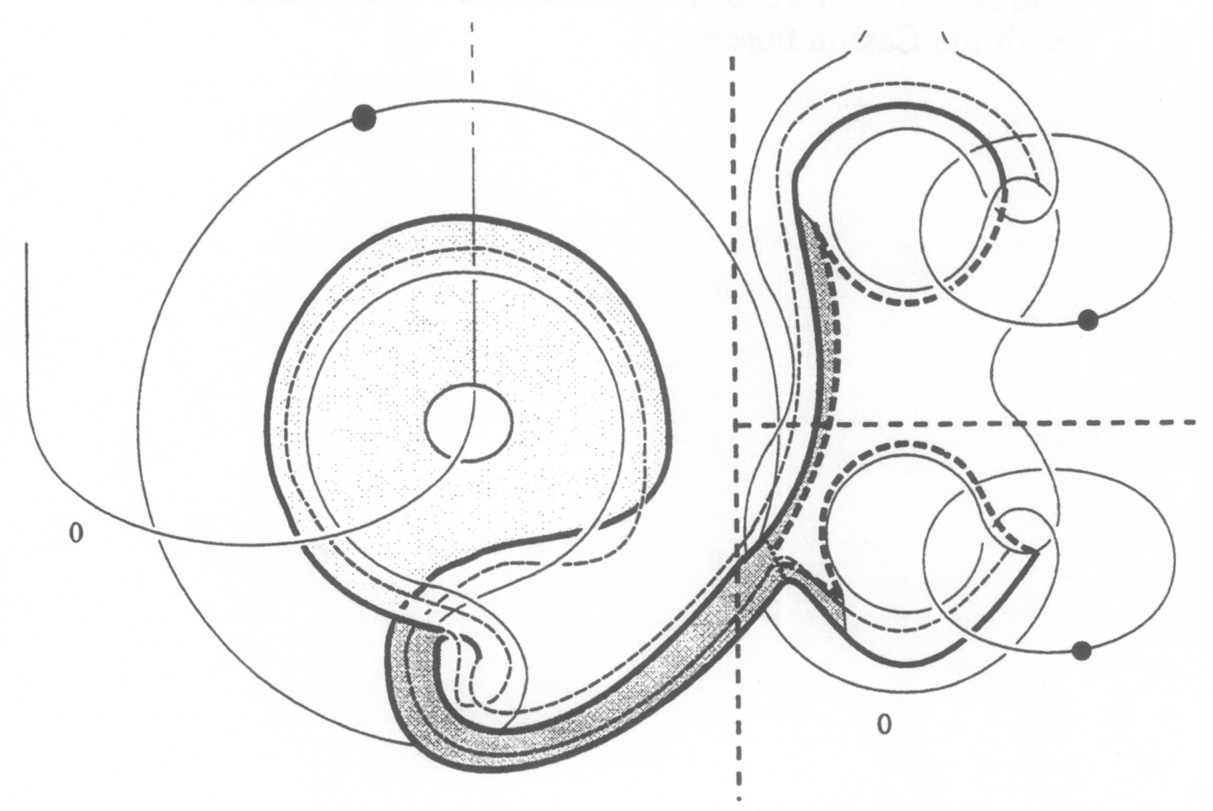

FIGURE 27. The slice on the left-hand side is near one of the intersection points between a pair of the fingers (see Figure 14). Those on the right are near the doubled caps of the surgered $F_{4}$ (see section 1.6.1). 


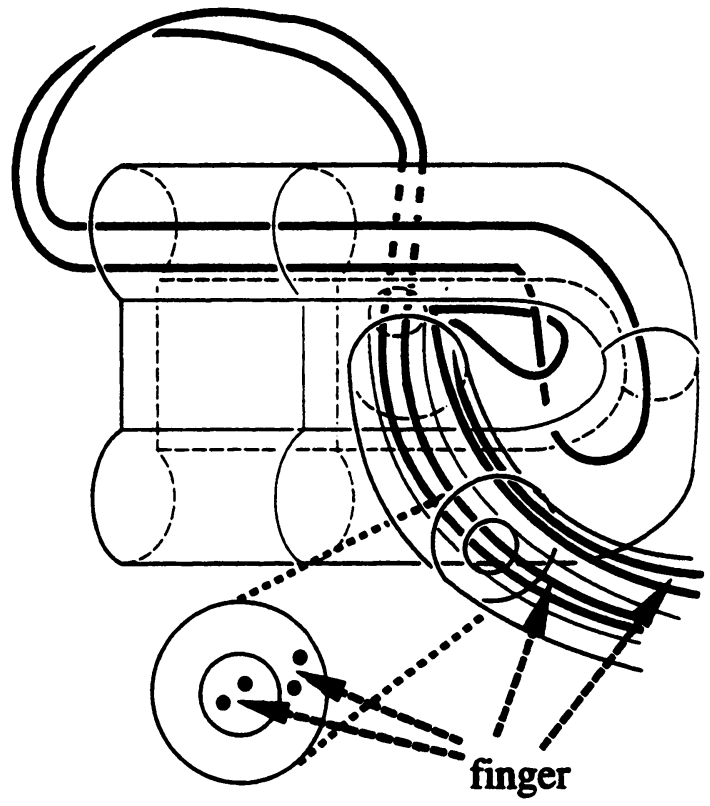

Figure 28. The added core surfaces, $F_{4}$ 's, are unlinked with the Casson fingers. 


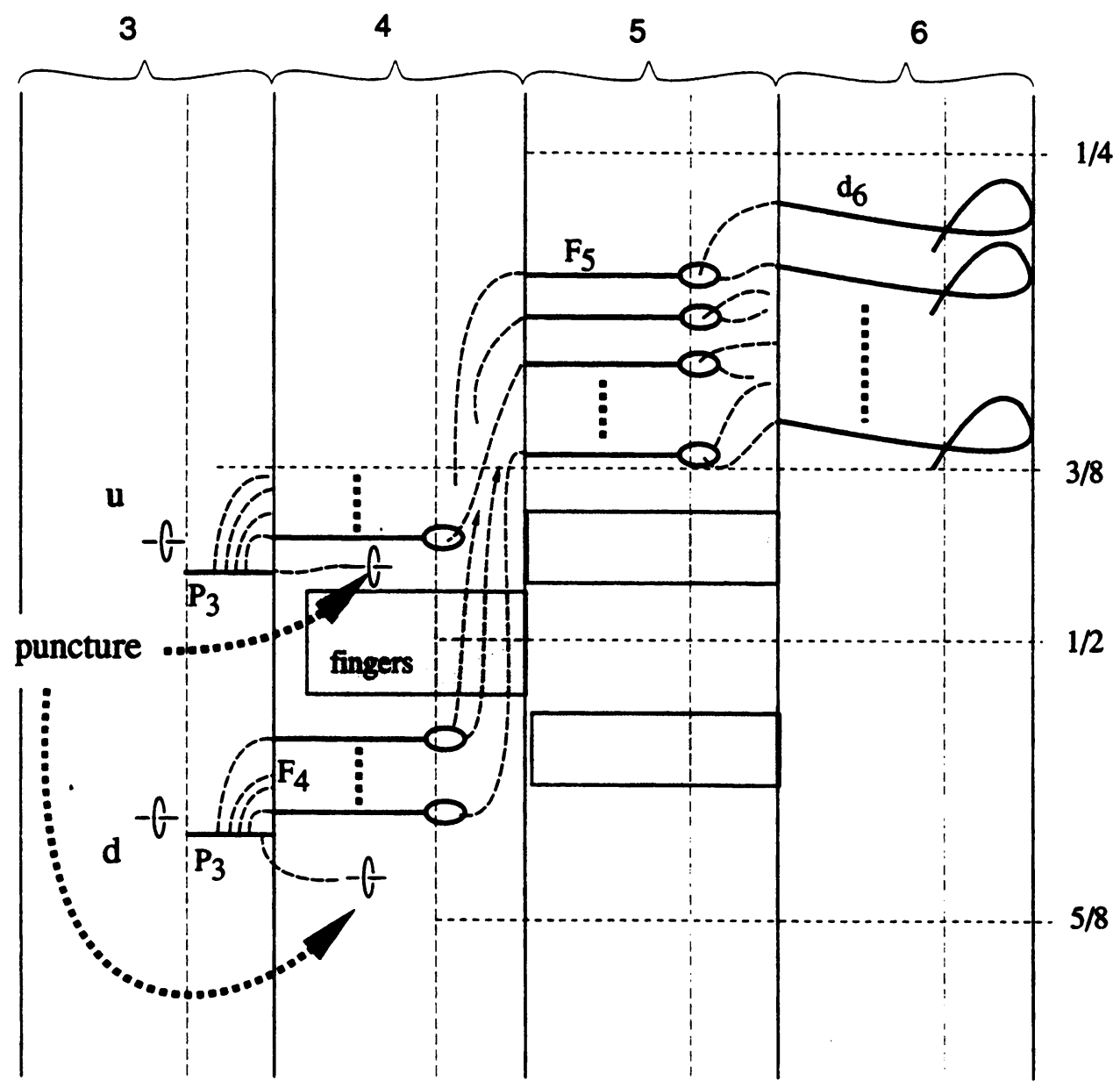

FIGURE 29. Capping towers for the punctures on a pair of fingers, as the one on the left-hand side of Figure 27. 


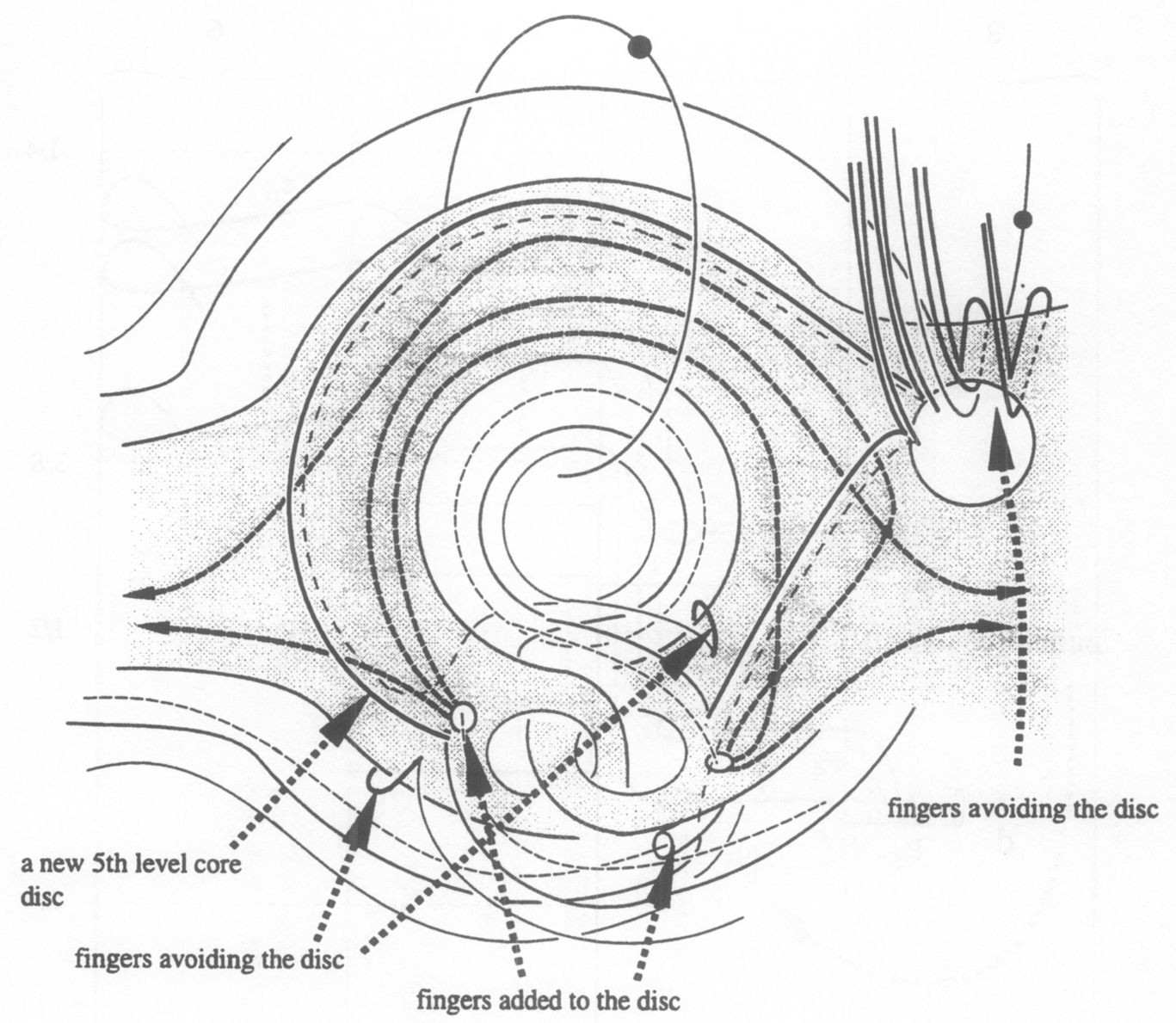

arcs added to the fingers:

Figure 30. A piece of the horizontal part of fifth-level core disc. 




Curves in the figures (b), (c) and (d) :

fingers on a 5 th level core disc:

fingers avoiding the caps:

loops:

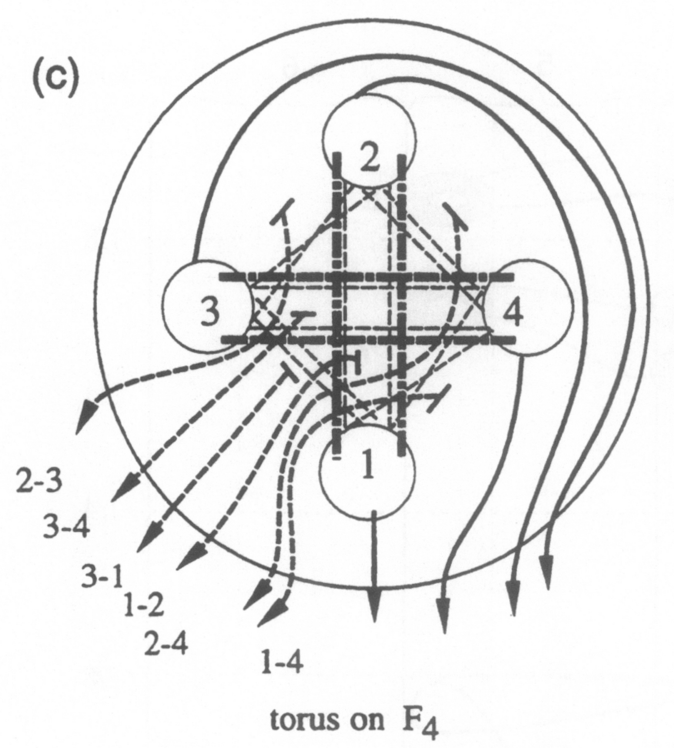

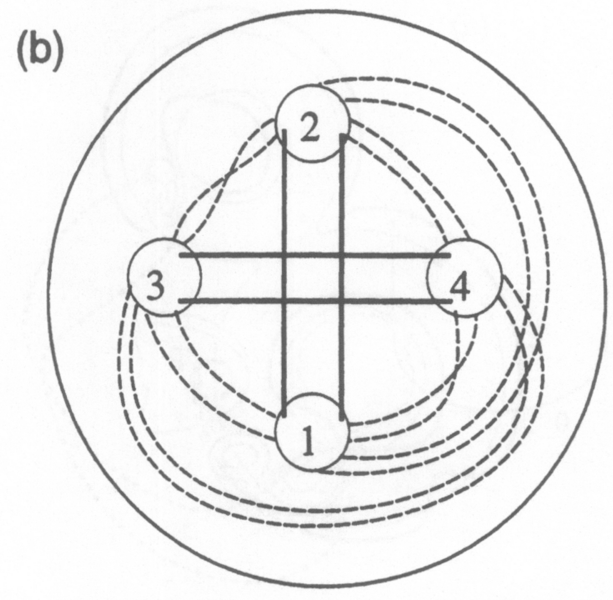

a characteristic torus or a torus on $\mathrm{F}_{3}$

(d)

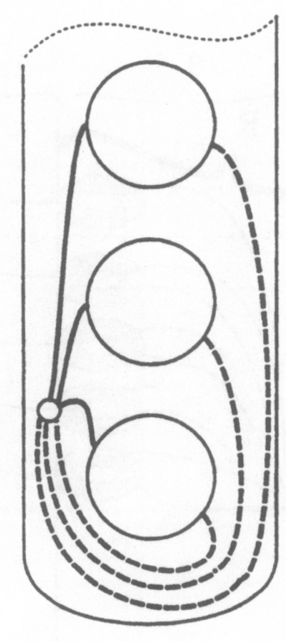

loops and fingers on $\mathrm{F}_{4}$

Figure 31 

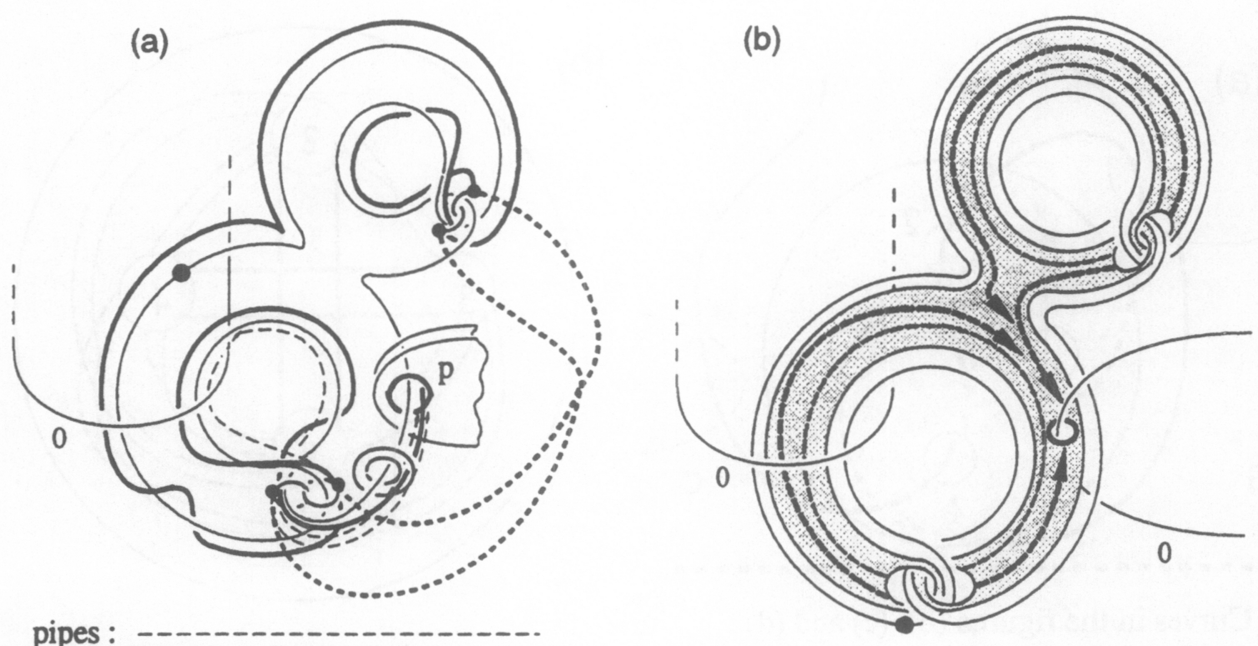

pipes :

added paths :

FIGURE 32

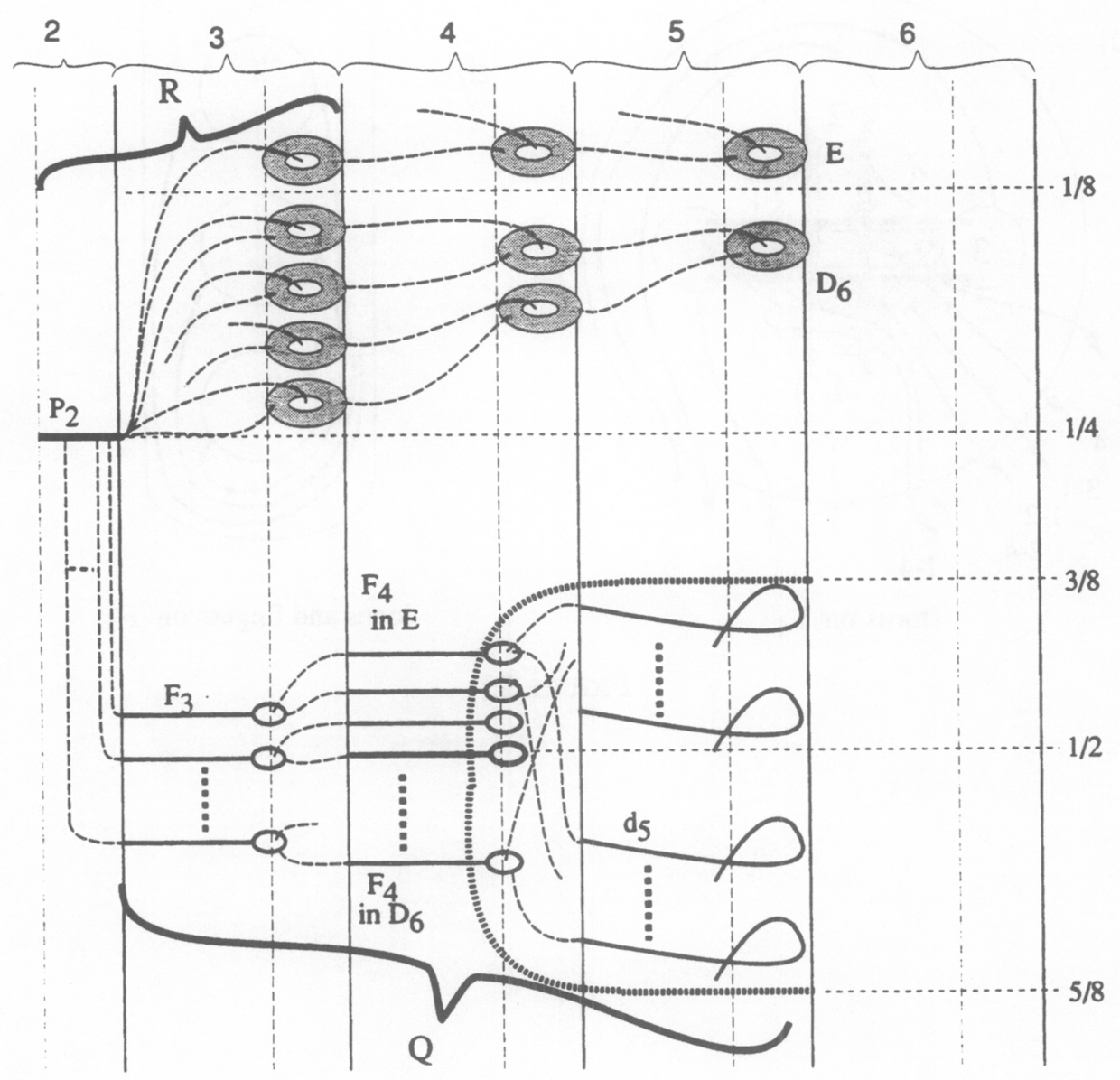

FIGURE 33. Duals to the sixth-level core discs: $D_{6}$ 's and $E$ 's. 


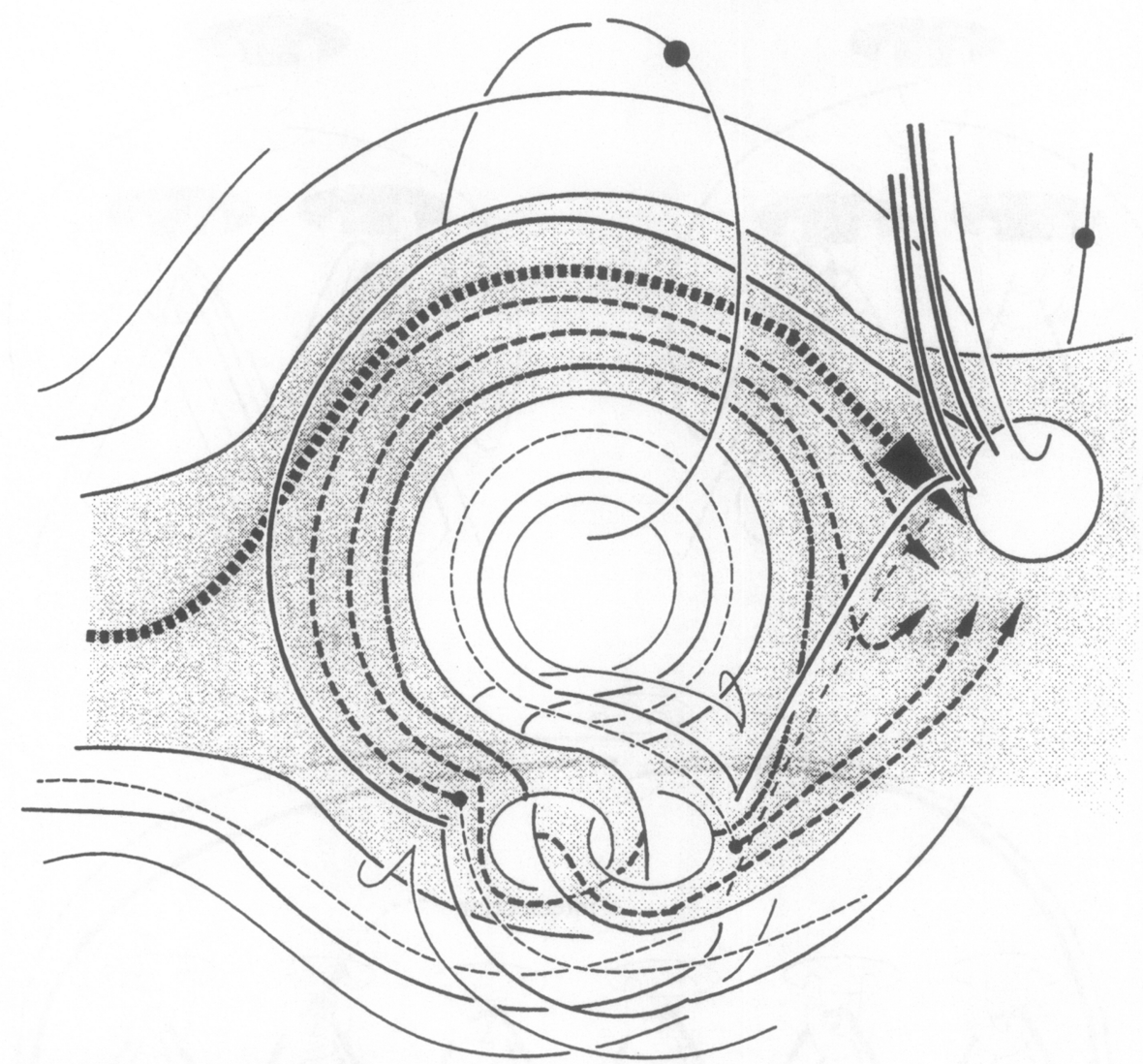

loop for the visible kink of the disc:

loops for intersecctions with parallel discs:

loops for other intersections:

Figure 34. Pieces of the loops near a $d_{5}$. 


$$
\begin{aligned}
& \overline{7} \\
& \overline{1808080}
\end{aligned}
$$




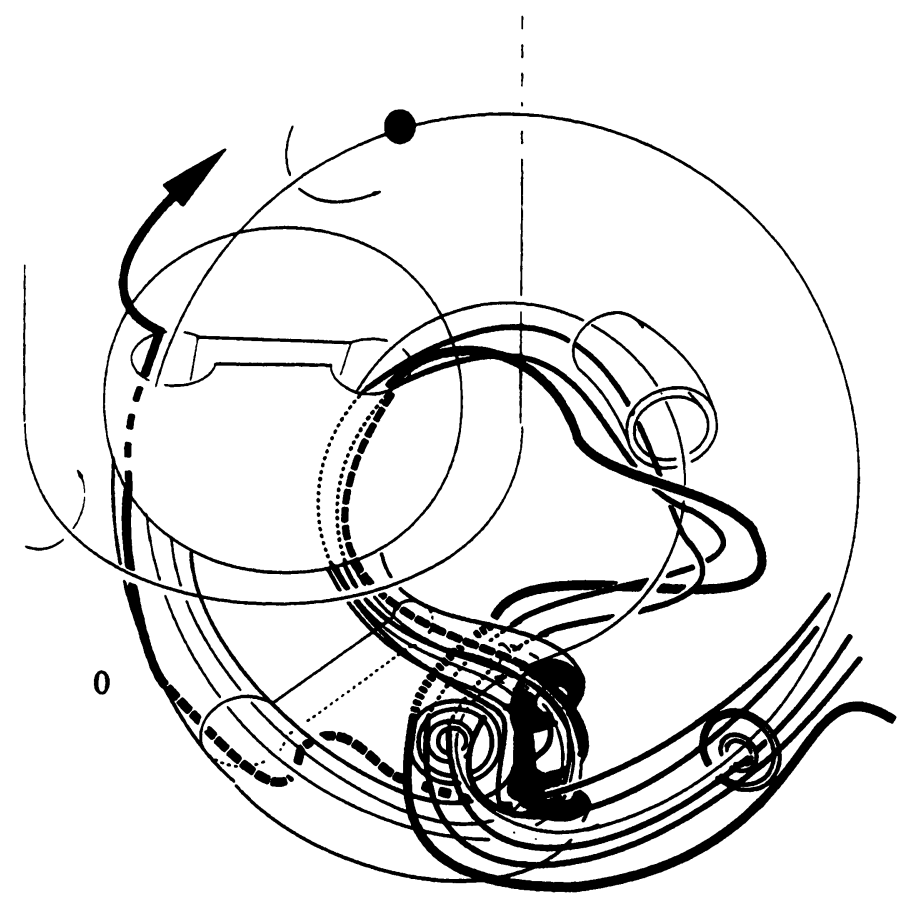

FIGURE 36
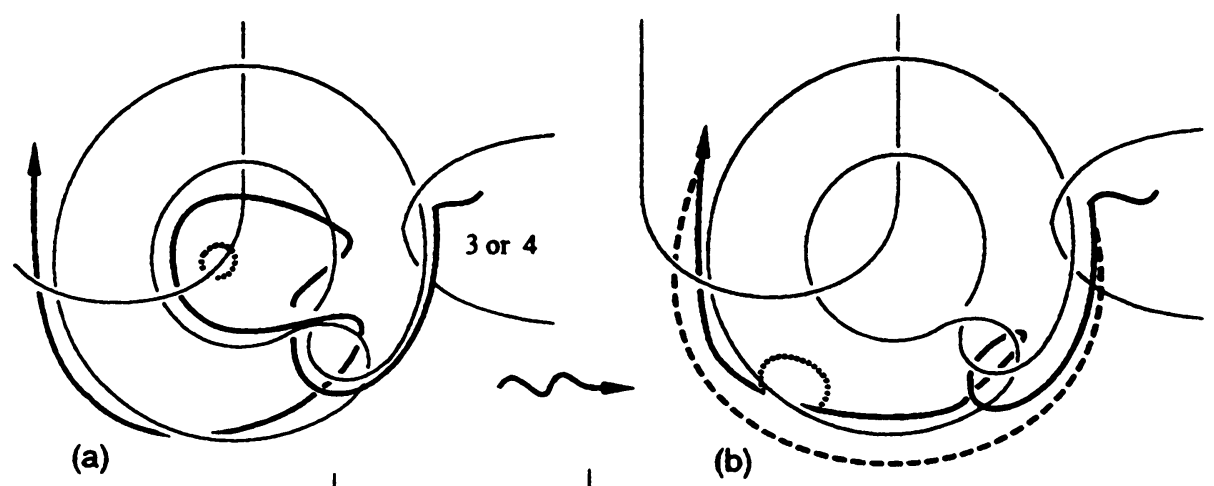

(a)

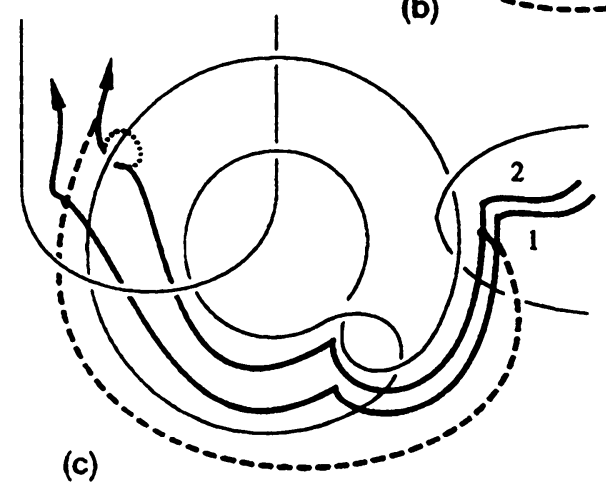

Figure 37 


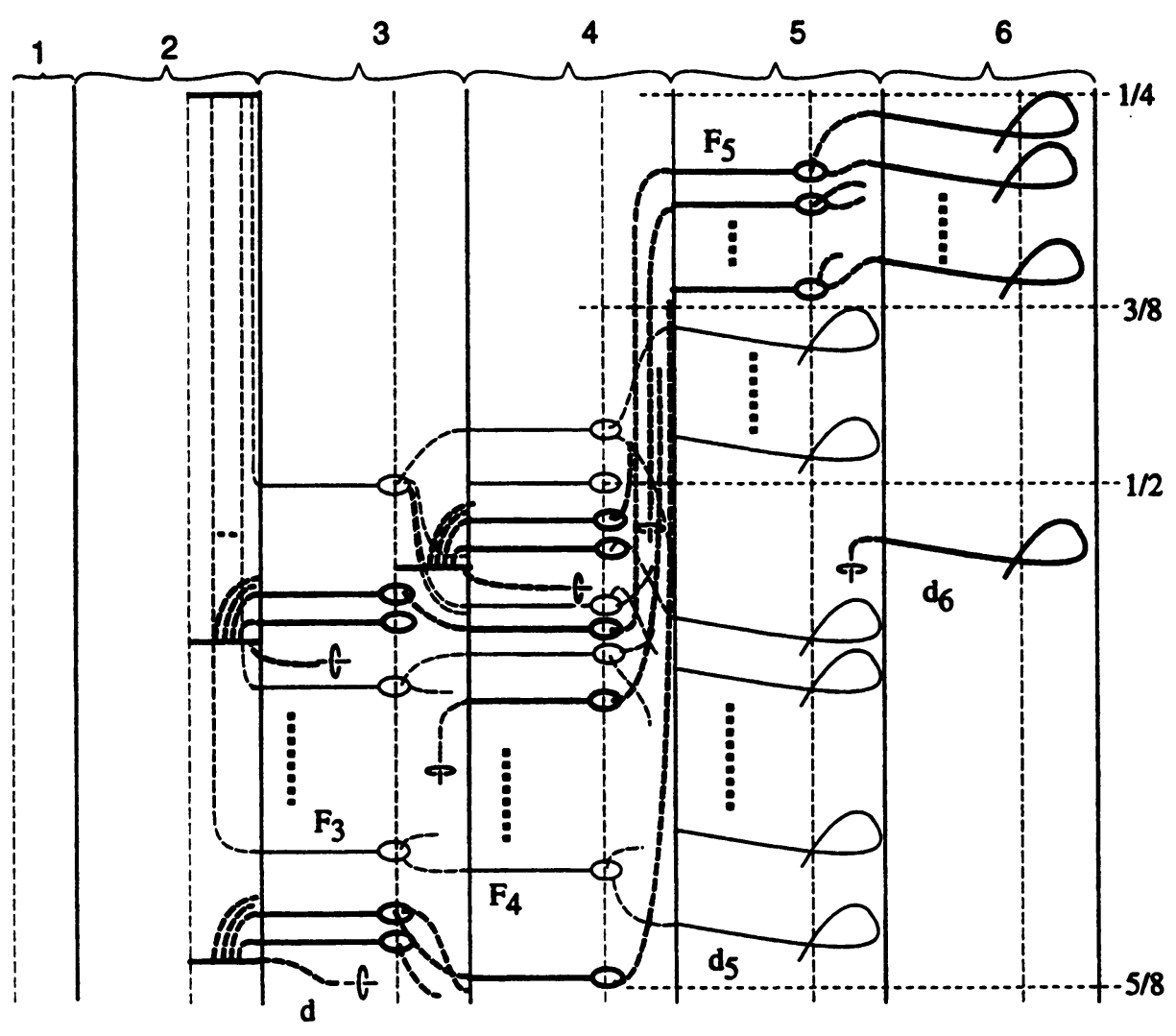

Figure 38. The $Q$ 's are drawn by thin lines. The bold lines denote discs which cap the punctures on the punctured discs bounded by the loops. 


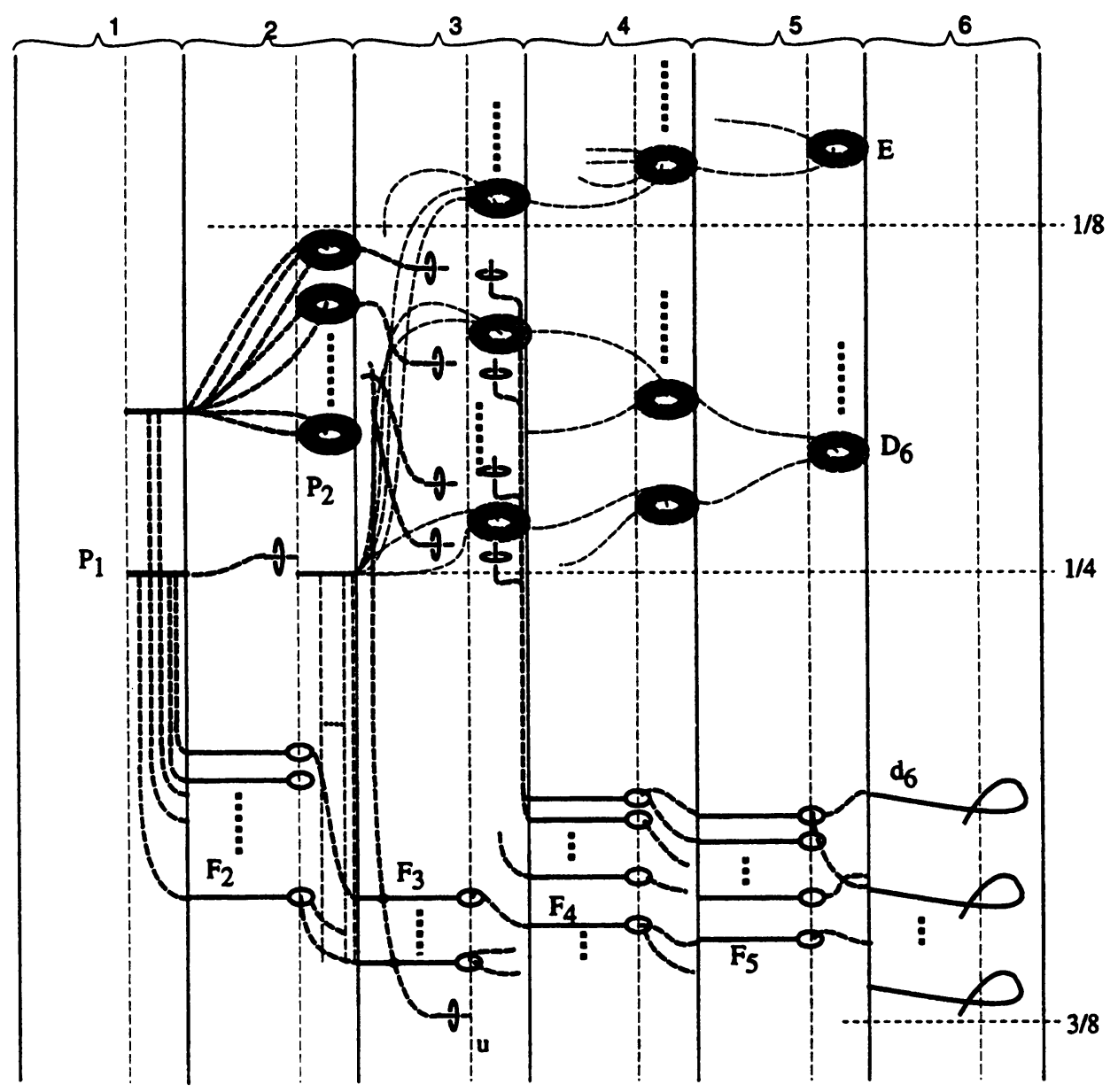

FIGURE 39. The $R$ 's are drawn by thin lines, and dises capping the punctures by bold lines. 


\section{REFERENCES}

[B1] Ż. Bižaca, $A$ handle decomposition of an exotic $\mathbf{R}^{4}$, J. Differential Geom. 39 (1994), Proc. Amer. Math. Soc. (to appear).

[B2] __, An explicit family of exotic Casson handles, preprint, 1993.

[C] A. Casson, Three lectures on new infinite construction in 4-dimensional manifolds (notes prepared by L. Guillou), A la Recherche de la Topologie Perdue (L. Guillou and A. Marin, eds.), Progr. in Math., vol. 62, Birkhäuser, Boston, 1986, pp. 201-244.

[D] S. Donaldson, An application of gauge theory to 4-dimensional topology, J. Differential Geom. 18 (1983), 279-315.

[DF] S. DeMichelis and M. Freedman, Uncountably many exotic $R^{4}$ 's in standard 4-space, J. Differential Geom. 35 (1992), 219-254.

[F1] M. Freedman, $A$ fake $S^{3} \times R$, Ann. of Math. (2) 110 (1979), 177-201.

[F2] —, The topology of 4-dimensional manifolds, J. Differential Geom. 17 (1982), 357-453.

[FQ] M. Freedman and F. Quinn, The topology of 4-manifolds, Princeton Univ. Press, Princeton, NJ, 1990.

[FT] M. Freedman and L. Taylor, A universal smoothing of four-space, J. Differential Geom. 24 (1986), 69-78.

[G1] R. Gompf, Three exotic $R^{4}$ 's and other anomalies, J. Differential Geom. 18 (1983), 317-328.

[G2] __, Infinite families of Casson handles and topological disks, Topology 23 (1984), 395-400.

[G3] _-, Smooth concordance of topologically slice knots, Topology 25 (1986), 353-373.

[G4] __, An infunite set of exotic $R^{4}$ 's, J. Differential Geom. 21 (1985), 283-300.

[G5] _ـ, An exotic menagerie, preprint, 1992.

[GS] R. Gompf and S. Singh, On Freedman's reimbedding theorems, Four-Manifold Theory (C. Gordon and R. Kirby, eds.), Contemp. Math., vol. 35, Amer. Math. Soc., Providence, R.I., 1984, pp. 277-310.

[Gr] M. Greenberg, Algebraic topology, Benjamin, New York, 1967.

[K1] R. Kirby, A calculus for framed links in $S^{3}$, Invent. Math. 45 (1978), 35-56.

[K2] __, The topology of 4 -manifolds, Lecture Notes in Math., vol. 1374, Springer-Verlag, Berlin and New York, 1989.

[T] C. Taubes, Gauge theory on asymptotically periodic 4-manifolds, J. Differential Geom. 25 (1987), 363-430.

Department of Mathematics and Computer Science, Clark Universtty, Worcester, MASSAChusetts 01610

Current address: Department of Mathematics, University of Texas, Austin, Texas 78712

E-mail address: bizacalmath. uteras . edu 